\title{
Cross-covariance isolate detect: a new change-point method for estimating dynamic functional connectivity
}

\author{
Andreas Anastasiou ${ }^{\mathrm{a}, *}$, Ivor Cribben $^{\mathrm{b}, *}$, Piotr Fryzlewicz ${ }^{\mathrm{c}}$ \\ ${ }^{a}$ Department of Mathematics and Statistics, University of Cyprus \\ ${ }^{b}$ Department of Accounting and Business Analytics, Alberta School of Business, University of Alberta \\ ${ }^{c}$ Department of Statistics, London School of Economics
}

\begin{abstract}
Evidence of the non stationary behavior of functional connectivity (FC) networks has been observed in task based functional magnetic resonance imaging (fMRI) experiments and even prominently in resting state fMRI data. This has led to the development of several new statistical methods for estimating this time-varying connectivity, with the majority of the methods utilizing a sliding window approach. While computationally feasible, the sliding window approach has several limitations. In this paper, we circumvent the sliding window, by introducing a statistical method that finds change-points in FC networks where the number and location of change-points are unknown a priori. The new method, called cross-covariance isolate detect (CCID), detects multiple change-points in the second-order (cross-covariance or network) structure of multivariate, possibly high-dimensional time series. CCID allows for change-point detection in the presence of frequent changes of possibly small magnitudes, can assign change-points to one or multiple brain regions, and is computationally fast. In addition, CCID is particularly suited to task based data, where the subject alternates between task and rest, as it firstly attempts isolation of each of the change-points within subintervals, and secondly their detection therein. Furthermore, we also propose a new information criterion for CCID to identify the change-points. We apply CCID to several simulated data sets and to task based and resting state fMRI data and compare it to recent change-point methods. CCID may also be applicable to electroencephalography (EEG), magentoencephalography (MEG) and electrocorticography (ECoG) data. Similar to other biological networks, understanding the complex network organization and functional dynamics of the brain can lead to profound clinical implications. Finally, the R package ccid implementing the method from the paper is available from CRAN.
\end{abstract}

Keywords: fMRI, Dynamic functional connectivity, Change-point analysis, Networks, Time varying connectivity

\footnotetext{
* Joint first authors

**Email address: anastasiou.andreas@ucy.ac.cy, cribben@ualberta.ca, p.fryzlewicz@lse.ac.uk
} 


\section{Introduction}

Functional connectivity (FC) is the undirected association between two or more functional magnetic resonance imaging (fMRI) time series. Evidence of the non stationary behavior of FC (or FC networks) has been observed in high temporal resolution electroencephalography (EEG) data, task based fMRI experiments (Fox et al., 2005, Debener et al., 2006, Cribben et al., 2012, 2013) and even prominently in resting state fMRI data (Delamillieure et al., 2010; Doucet et al., 2012, Cribben \& Yu, 2017; Ofori-Boateng et al., 2020). By estimating a FC network across the entire experimental time course, the resulting FC network is simply an average of the changing connectivity structures. While this is convenient for estimation and computation purposes, as it keeps the FC network estimation from becoming too complex, it presents a simplified version of a highly integrated and dynamic phenomenon.

In order to estimate this time varying phenomenon (or dynamic FC as it is widely known), many research papers first considered a sliding (or moving) window. These approaches begin at the first time point, then a block of a fixed number of time points (the window) are selected and all the data points within the block are used to estimate the FC. The window is then shifted a certain number of time points (overlapping or non-overlapping) and the FC is estimated on the new data. By shifting the window to the end of the experimental time course, researchers can estimate the dynamic FC. Chang \& Glover (2010), Kiviniemi et al. (2011), Hutchison et al. (2013b) and Leonardi et al. (2013) investigated the dynamic functional FC in resting-state data using a moving-window approach, based on a time-frequency coherence analysis with wavelet transforms, an independent component analysis, a correlation analysis, and a principal component analysis, respectively. Allen et al. (2014), Handwerker et al. (2012), Jones et al. (2012) and Sakoğlu et al. (2010) considered a group independent component analysis (Calhoun et al. 2001) to decompose multisubject resting-state data into functional brain regions, and a moving-window and $k$-means clustering of the windowed correlation matrices to study whole brain dynamic FC networks. While the sliding window approach is computationally feasible, it also has limitations (Hutchison et al. 2013a). For example, the choice of window size is crucial and sensitive, as different window sizes can lead to quite different FC patterns. Another disadvantage is that equal weight is given to all $k$ neighbouring time points and 0 weight to all the others (Lindquist et al., 2014). Other methods have also been proposed that do away with the sliding window. For example, Zhang et al. (2014) proposed the dynamic Bayesian variable partition model that estimates and models multivariate dynamic functional interactions using a unified Bayesian framework.

Change-point methods have also been considered. There exists an extensive literature and a long history on change-points. The most widely discussed problems have been concerned with finding multiple change-points in univariate time series (Inclan \& Tiao, 1994, Chen \& Gupta, 1997). Recently, the multiple change-point detection problem in multivariate time series has received some attention especially in non-stationary practical problems (Fan et al. 2011$)$. High dimensional time series change-point detection 
problems are the obvious but by no means straightforward extension of the univariate case. To detect changes in the covariance matrix of a multivariate time series, Aue et al. (2009) introduced a method using a nonparametric CUSUM type test, Dette \& Wied (2016) proposed a test where the dimension of the data is fixed while Kao et al. (2018) considered the case where the dimension of the data increases with the sample size (they also investigated change-point analysis based on Principal Component Analysis). Sundararajan \& Pourahmadi (2018) proposed a new method for detecting multiple change-points in the covariance structure of a multivariate piecewise-stationary process.

In other related work, Barnett \& Onnela (2016) considered a method for detecting change-points in correlation networks that, unlike previous change-point detection methods designed for time series data, requires minimal distributional assumptions. Gibberd \& Nelson(2014) studied the consistency properties of a regularised estimator for the simultaneous identification of both change-points and the graphical dependency structure in multivariate time series. The first comprehensive treatment of high dimensional time series factor models with multiple change-points in their second-order structure is put forward by Barigozzi et al. (2018). Li et al. (2019) considered multiple structural breaks in large contemporaneous covariance matrices of high dimensional time series satisfying an approximate factor model. Cho \& Fryzlewicz (2015) segmented the multivariate time series into partitions based on the second-order structure. Within neuroscience, Cribben et al. (2012, 2013) put forward the Dynamic Connectivity Regression method for detecting multiple changepoints in the precision matrices (or undirected graph) from a multivariate time series. Schröder \& Ombao (2019) introduced FreSpeD that employed a multivariate cumulative sum (CUSUM)-type procedure to detect change-points in autospectra and coherences for multivariate time series. Their method allows for the segmentation of the multivariate time series but also for the direct interpretation of the change in the sense that the change-point can be assigned to one or multiple time series (or Electroencephalogram channels) and frequency bands. Kirch et al. (2015) considered the At Most One Change setting and the epidemic setting (two change-points, where the process reverts back to the original regime after the second change-point) and provide some theoretical results. Cribben \& Yu (2017) introduced the Network Change Point Detection method that uses an eigen-space based statistic for testing the community structures changes in stochastic block model sequences. More recently, Kundu et al. (2018) proposed a fully automated two-stage approach which pools information across multiple subjects to estimate change-points in functional connectivity, while Dai et al. (2019) developed a formal statistical test for finding change-points in time series associated with FC. Finally, Ofori-Boateng et al. (2020) introduced a new method that firstly presents each network snapshot of fMRI data as a linear object and finds its respective univariate characterization via local and global network topological summaries and then adopts a change-point detection method for (weakly) dependent time series based on efficient scores.

All of these methods have limitations. The most obvious is that they all use binary segmentation (BS) 
to identify multiple change-points. BS, while computationally feasible, is not optimal as it searches for a single change-point at a time. Hence, BS struggles to find multiple change-points in task based fMRI experiments, where the subject alternates between two states, rest and task, as the network structure for any two segments in the data split by a change-point is very similar. Furthermore, it is difficult for BS to find frequent changes of possibly small magnitudes. In addition, many of the previous methods (Cribben et al. 2012, 2013, Schröder \& Ombao, 2019, Kirch et al. 2015, Dai et al. 2019) can only consider a relatively low number (up to $p=30$ ) of time series from either the channels or brain regions. Hence, their ability to detect whole brain connectivity change-points is impeded. In order to include a large number of time series, other methods (Cribben \& Yu, 2017; Ofori-Boateng et al., 2020; Ondrus et al., 2021) perform a dimension reduction technique, such as singular value decomposition or summarize the network using graph summary statistics, to the data to make the problem more computationally feasible, but may lose information from the network structure, and hence the change-points therein, in this process.

In this paper, we introduce a new method, called cross-covariance isolate detect (CCID), to detect multiple change-points in the second-order structure of multivariate, possibly high-dimensional time series. The second-order structure can also be referred to as the dependence structure, the cross-covariance structure or the network structure, between the time series. CCID begins by first converting the multivariate time series (or regions of interest time series) into local wavelet periodograms and cross-periodograms. To detect the change-points, CCID aggregates across the multivariate time series through the $L_{2}$ or the $L_{\infty}$ metric and then uses a scaled CUSUM statistic to decide whether a candidate is indeed a change-point or not, based on whether the relevant scaled CUSUM value is larger than an appropriately chosen threshold. Furthermore, we propose a new information criterion for CCID as an alternative approach to the threshold method for the change-point detection problem. CCID has the following unique and important attributes. First, CCID uses the Isolate-Detect principle (Anastasiou \& Fryzlewicz, 2021) to find the multiple change-points. CCID works by first isolating each of the change-points within subintervals and then secondly detecting them within these subintervals. For general data sets we consider in this work, we find that CCID performs as well as, if not better than, the previous methods that use BS. However, for our simulations, where the subject alternates between two states and for our task based fMRI experiment, CCID clearly outperforms the BS methods. This is due to CCID's ability to isolate the change-points between tasks while BS looks to partition the data into two intervals which is particularly difficult given the similarity between any two partitions (for this particular data type) and the fact that BS tends not to handle frequent change-point scenarios well. Second, CCID has the ability to find change-points that are very close to one another, which provides more insight into functional dynamics of the brain. Third, after detecting the change-points, CCID can assign them to one or multiple brain regions. Fourth, CCID is computationally fast. Fifth, CCID may also be applicable to electroencephalography (EEG), magentoencephalography (MEG) and electrocorticography (ECoG) data. 
Finally, the $\mathrm{R}$ package ccid implementing the method from the paper is available from CRAN.

The remainder of this paper is organized as follows. We introduce our new method, CCID, in Section 2. We describe our simulated data and our fMRI data in Section 3 . The performance of the proposed method on both the simulated and real data is detailed in Section 4. A discussion of the limitations of the method and future work is described in Section 5 . Finally, we conclude in Section 6 and provide details on the R package ccid in Section 7

\section{Materials and methods}

Let $\mathbf{X} \in \mathbb{R}^{T \times p}$ be a multivariate time series with $T$ time points and $p$ regions of interest (ROIs: or data sequences). We assume that each univariate time series within $\mathbf{X}$ follows a Gaussian distribution. Smith et al. (2011) conclude that the Gaussian assumption is valid on good-quality fMRI data. We show how crosscovariance isolate detect (CCID) can be adapted to detect multiple change-points in the second-order (or cross-covariance) structure of multivariate, possibly high-dimensional time series. The second-order structure can also be referred to as the dependence structure, the cross-covariance structure or the network structure, between the time series. CCID first constructs wavelet-based local periodogram and cross-periodogram sequences from $\mathbf{X}$, to which we apply the isolate-detect principle (Anastasiou \& Fryzlewicz, 2021) to find multiple change-points in the second order structure of $\mathbf{X}$.

\subsection{Wavelets}

A wavelet is a wave-like oscillation. Its amplitude begins at zero, increases (decreases), and then decreases (increases) back to zero. In wavelet theory, the wavelet correlates with another signal if it is of similar frequency at the location overlapping with the support of the wavelet. As a mathematical tool, wavelets can be used to extract information from many different types of data. We use wavelets in this work to transform the problem of detecting change-points in the second-order structure of the process to the expectations of the wavelet cross-periodograms and to "whiten" the data. For a detailed review of wavelets we refer readers to Daubechies (1992) and Vidakovic (2009).

\subsection{Locally Stationary Wavelet model}

By way of introduction, we consider Haar wavelets (the simplest example of a wavelet system) which are defined as

$$
\psi_{i, k}^{H}=2^{i / 2} \mathbb{I}\left(0 \leq k \leq 2^{-i-1}\right)-2^{i / 2} \mathbb{I}\left(2^{-i-1} \leq k \leq 2^{-i}-1\right),
$$

where $i \in\{-1,-2, \ldots\}$ and $k \in \mathbb{Z}$ denote scale and location parameters, respectively. Small negative values of the scale parameter $i$ denote fine scales where the wavelet vectors are the most localized and oscillatory, while large negative values denote coarse scales with longer, less oscillatory wavelet vectors. With such 
wavelets as building blocks, we introduce the $p$-variate Locally Stationary Wavelet (LSW: Cho \& Fryzlewicz, 2015) model as follows:

Definition 1. The $p$-variate LSW process $\left\{\mathbf{X}_{t, T}=\left(X_{t, T}^{(1)}, \ldots, X_{t, T}^{(p)}\right)^{\prime}\right\}_{t=0}^{T}$ for $T=1,2, \ldots$, is a triangular stochastic array with the following representation:

$$
X_{t, T}^{(j)}=\sum_{i=-\infty}^{-1} \sum_{k=-\infty}^{\infty} W_{i}^{(j)}(k / T) \psi_{i, t-k} \xi_{i, k}^{(j)}, \text { for each } j=1, \ldots, p
$$

where $\xi_{i, k}=\left(\xi_{i, k}^{(1)}, \xi_{i, k}^{(2)}, \ldots, \xi_{i, k}^{(p)}\right)^{\prime}$ are independently generated from multivariate normal distributions $N_{p}\left\{\mathbf{0}, \boldsymbol{\Sigma}_{i}(k / T)\right\}$, with $\Sigma_{i}^{(j, j)}(k / T)=1$ and

$$
\operatorname{cov}\left(\xi_{i, k}^{(j)}, \xi_{i^{\prime}, k^{\prime}}^{(l)}\right)= \begin{cases}\delta_{i, i^{\prime}} \delta_{k, k^{\prime}} \Sigma_{i}^{(j, j)}(k / T)=\delta_{i, i^{\prime}} \delta_{k, k^{\prime}} & \text { when } j=l \\ \delta_{i, i^{\prime}} \delta_{k, k^{\prime}} \Sigma_{i}^{(j, l)}(k / T) & \text { when } j \neq l\end{cases}
$$

The Kronecker delta function $\delta_{i, i^{\prime}}$ is 1 when $i=i^{\prime}$ and 0 otherwise. For each $i$ and $j, l=1, \ldots, p$, the functions $W_{i}^{(j)}(k / T):[0,1] \rightarrow \mathbb{R} \geq 0$ and $\Sigma_{i}^{(j, l)}(k / T):[0,1] \rightarrow \mathbb{R}$ are piecewise-constant with an unknown number of change-points. The autocovariance and cross-covariance functions of $\mathbf{X}_{t, T}$ approximately inherit the piecewise-constant property of $W_{i}^{(j)}($.$) and \Sigma_{i}^{(j, l)}($.$) with almost identical change-point locations. See$ Cho \& Fryzlewicz (2015) for details.

\subsection{Wavelet periodograms and cross-periodograms}

We now construct appropriate wavelet-based local periodogram sequences from the $p$-variate LSW time series $\mathbf{X}_{t, T}$. With the empirical wavelet coefficients at scale $i$ denoted by $w_{i, t, T}^{(j)}=\sum_{u} X_{u, T}^{(j)} \psi_{i, t-u}$ for each $X_{t, T}^{(j)}, j=1, \ldots, p$, the wavelet periodogram of $X_{t, T}^{(j)}$ and the wavelet cross-periodogram between $X_{t, T}^{(j)}$ and $X_{t, T}^{(l)}$ at scale $i$ are defined as

$$
\begin{array}{r}
I_{i, t, T}^{(j, j)} \equiv I_{i, t, T}^{j}=\left(w_{i, t, T}^{(j)}\right)^{2} \\
I_{i, t, T}^{(j, l)}=w_{i, t, T}^{(j)} \cdot w_{i, t, T}^{(l)}
\end{array}
$$

respectively. As $X_{t, T}^{(j)}$ is Gaussian, $w_{i, t, T}^{(j)}$ is also Gaussian, hence each $I_{i, t, T}^{(j, j)}$ follows a scaled $\chi_{1}^{2}$ distribution.

For a multivariate LSW process $\mathbf{X}_{t, T}$, its theoretical (population) local autocovariance and cross-covariance functions are defined by

$$
\begin{array}{r}
c_{T}^{(j, j)}(z, \tau)=c_{T}^{(j)}(z, \tau)=\operatorname{cov}\left(X_{\lfloor z T\rfloor, T}^{j}, X_{\lfloor z T\rfloor+\tau, T}^{j}\right) \\
c_{T}^{(j, l)}(z, \tau)=\operatorname{cov}\left(X_{\lfloor z T\rfloor, T}^{j}, X_{\lfloor z T\rfloor+\tau, T}^{l}\right),
\end{array}
$$

In the multivariate LSW model, Cho \& Fryzlewicz $(2015)$ showed that there is an asymptotic one-to-one 
correspondence between these theoretical local autocovariance and cross-covariance functions, the population wavelet periodograms and cross-periodograms, and the expectations of wavelet periodograms and cross-periodograms. Specifically, there exists an asymptotic one-to-one correspondence for any pair of $X_{t, T}^{(j)}$ and $X_{t, T}^{(l)}$ between the following quantities: the autocovariance and cross-covariance functions $c_{T}^{(j)}(z, \tau)$, $c_{T}^{(l)}(z, \tau)$ and $c_{T}^{(j, l)}(z, \tau)$ at lags $\tau=0,1, \ldots$, piecewise-constant functions $W_{i}^{(j)}(z)^{2}, W_{i}^{(l)}(z)^{2}$ and $\Sigma_{i}^{(j, l)}(z)$ and the expectations of wavelet periodograms and cross-periodograms $\mathbb{E} I_{i, t, T}^{(j)}, \mathbb{E} I_{i, t, T}^{(l)}$ and $\mathbb{E} I_{i, t, T}^{(j, l)}$ at scales $i=-1,-2, \ldots$. Therefore, the change-points in the second order structure of the multivariate time series $\mathbf{X}_{t, T}$ can be detected from the wavelet periodograms and cross-periodograms at multiple scales.

We now focus on the wavelet periodogram $I_{i, t, T}^{(j)}$ and the cross-periodogram $I_{i, t, T}^{(j, l)}$, and use them as the inputs into the CCID algorithm. We firstly note that $\mathbb{E} I_{i, t, T}^{(j)}$ are piecewise-constant except for negligible biases around the change-points. Since $I_{i, t, T}^{(j, l)}$ does not follow a multiplicative model, we consider instead the following alternative:

$$
\tilde{I}_{i, t, T}^{(j, l)}=\left(w_{i}^{(j)}-\operatorname{sign}\left(\operatorname{côr}\left(w_{i, t, T}^{(j)}, w_{i, t, T}^{(l)}\right)\right) \cdot w_{i, t, T}^{(l)}\right)^{2}
$$

where côr is the sample correlation computed separately on each current segment (data between changepoints). This is a multiplicative model but results in no loss of information about any change-points, see Cho \& Fryzlewicz (2015).

There are four main advantages for using wavelets (the LSW model) in our CCID model for the purpose of change-point detection. First, there is one-to-one correspondence between the auto/cross-covariance structure of a time series, and its wavelet spectrum, or alternatively its (population) wavelet cross-periodograms. The (population) wavelet cross-periodogram representation, enabled by using the wavelet model, is the most convenient of the three for the purpose of change-point detection. The reason is that the natural estimator of the population wavelet (cross-)periodogram is the empirical wavelet (cross-)periodogram, which is a scaled $\chi^{2}$ distributed quantity under Gaussianity (and follows a general multiplicative model under other distributions). This makes it particularly suited for segmentation (=change-point detection) as a great deal is known in the literature (Inclan \& Tiao, 1994, Cho \& Fryzlewicz, 2015) about segmenting multiplicative-model data sequences of the form present in the empirical wavelet (cross-)periodograms. Second, a known difficult issue arising in time series segmentation is the presence of autocorrelation in the data, which can 'fool' changepoint detectors as natural fluctuations in the time series due to autocorrelations can mask as change-points, and vice versa. The use of wavelets (we need to take a wavelet transform of the data in order to compute the empirical cross-periodograms) are helpful here due to their well-known whitening property (Vidakovic, 2009), whereby they tend to reduce positive autocorrelation in time series, hence making the change-point detection problem easier. Third, the whole array of the wavelet periodograms at all scales are easily and rapidly computable via the wavelet transform. Fourth, the normality assumption in LSW is reasonable for high quality fMRI data. 


\subsection{Model specification}

With $p$ being the dimensionality of the initial given time series, we assume the following multiplicative model for the periodograms $I_{i, t, T}^{(j)}$ and cross periodograms $\tilde{I}_{i, t, T}^{(j, l)}$ for $i=1, \ldots, p$, and $d=J p(p+1) / 2$ (assuming $J$ scales), by transforming our data to periodograms and cross periodograms, which we denote as $\mathbf{Y}$ :

$$
Y_{t, T}^{(k)}=\sigma_{t, T}^{(k)}\left(\left(Z_{t, T}\right)^{(k)}\right)^{2}, \quad t=1,2, \ldots, T, \quad k=1,2, \ldots, d,
$$

where $Y_{t, T}^{(k)}$ are the observed data related to the $k^{t h}$ data sequence, $\sigma_{t, T}^{(k)}$ is a piecewise-constant mean function and $Z_{t, T}^{(k)}$ is a sequence of (possibly) auto-correlated standard normal random variables. This means that $Y_{t, T}^{(k)}$ is a scaled $\chi_{1}^{2}$-random variable, as the empirical wavelet periodogram is a squared zero-mean normal variables Cho \& Fryzlewicz, 2012), with $\mathbb{E}\left(Y_{t, T}^{(k)}\right)=\sigma_{t, T}^{(k)}>0$. Our purpose is to detect the number and the location of changes in the mean of $\sigma_{t, T}^{(k)}$, for any $k \in\{1,2, \ldots, d\}$, with each change-point being possibly shared by more than one data sequence. We only consider the finest scale of $i=-1$ used in the wavelet transformation as fMRI data typically only have lag 1 autocorrelation (Fiecas et al., 2017; Arbabshirani et al. 2014). Hence, $d=p(p+1) / 2$.

In order to detect the change-points we use the following statistic:

$$
\begin{aligned}
\tilde{Y}_{s, e}^{b,(k)}=\left(\frac{1}{e-s+1} \sum_{t=s}^{e} Y_{t, T}^{(k)}\right)^{-1} \mid \sqrt{\frac{e-b}{(b-s+1)(e-s+1)}} \sum_{t=s}^{b} Y_{t, T}^{(k)} \\
-\sqrt{\frac{b-s+1}{(e-b)(e-s+1)}} \sum_{t=b+1}^{e} Y_{t, T}^{(k)} \mid,
\end{aligned}
$$

where $1 \leq s \leq b<e \leq T$. The statistic is a (scaled) CUSUM statistic, where we divide by the sample mean of the observations $Y_{t, T}^{(k)}$ with $t \in[s, e]$. This is necessary in multiplicative settings such as (7) in order to make the results independent of the magnitude of $\sigma_{t, T}^{(k)}$. Essentially, the CUSUM statistic compares the means of the data to the left and to the right of each postulated change-point location $b$. To obtain a candidate change-point on any interval $[s, e]$, we maximise the CUSUM statistic over $b$ : this is where the (scaled) difference in means is the largest, so this will be the likeliest candidate for a change-point. In addition, we note that due to (7), the statistic in (8) is positive. More information on 8 can be found in Inclan \& Tiao (1994) and Cho \& Fryzlewicz (2015).

\section{5. $C C I D$}

Our new method, CCID, proceeds as follows. We describe the Isolate-Detect Anastasiou \& Fryzlewicz, 2021) segmentation algorithm illustrated in Figure 1. The basic idea is that for the $d$ observed data sequences of length $T$ each, and with $\lambda_{T}$ a suitably chosen positive integer, Isolate-Detect (ID) first creates two ordered sets of $K=\left\lceil T / \lambda_{T}\right\rceil$ right- and left-expanding intervals. We collect these intervals in the ordered set 
$S_{R L}=\left\{R_{1}, L_{1}, R_{2}, L_{2}, \ldots, R_{K}, L_{K}\right\}$. Then, for each point $b$ in the interval $R_{1}$ and for each $k=1,2 \ldots, d$, we calculate the values of $\tilde{Y}_{1, \lambda_{T}}^{b,(k)}$ as in equation (8). As already mentioned, we aggregate the information of each of the $d$ data sequences using either the $L_{2}$ or the $L_{\infty}$ metric. For the $L_{2}$ approach for CCID, we define

$$
\tilde{U}_{s, e}^{b, L_{2}}=\frac{1}{\sqrt{d}} \sqrt{\sum_{k=1}^{d}\left(\tilde{Y}_{s, e}^{b,(k)}\right)^{2}}, \quad b \in[s, e),
$$

and we set $b^{*}:=\underset{b \in R_{1}}{\operatorname{argmax}} \tilde{U}_{1, \lambda_{T}}^{b, L_{2}}$. If this value exceeds a certain threshold, denoted by $\zeta_{T}^{L_{2}}$, then it is taken as a change-point. If not, then the process tests the next interval in $S_{R L}$, which in this case would be $L_{1}$. Upon detection the ID methodology makes a new start from the end-point (or start-point, respectively) of the right- (or left-, respectively) expanding interval where the detection occurred. At some point we find an interval $[s, e]$ that does not contain any other change-points because at each stop we expand the intervals by $\lambda_{T}$ which is smaller than the minimum distance in $(9)$ between two change-points; this is where isolation stems from as well, which in order to be ensured, the expansion parameter $\lambda_{T}$ can be taken to be as small or equal to 1 . If now $\lambda_{T}>1$, then isolation is guaranteed with high probability. Theoretically for large $T$, the chosen value for $\lambda_{T}$ (this typically is small; see Anastasiou \& Fryzlewicz (2021) for more details) is guaranteed to be smaller than the minimum distance $\delta_{T}$ (which is unknown but has to grow with $T$ ) between two consecutive change-points.

The $L_{\infty}$ approach works similarly. However, instead of using the Euclidean distance in (9), we use

$$
\tilde{U}_{s, e}^{b, L_{\infty}}=\max _{k \in\{1,2, \ldots, d\}}\left\{\left|\tilde{Y}_{s, e}^{b,(k)}\right|\right\}, \quad b \in[s, e)
$$

and we set $\tilde{b}:=\underset{b \in R_{1}}{\operatorname{argmax}} \tilde{U}_{1, \lambda_{T}}^{b, L_{\infty}}$. If this value exceeds a certain threshold, denoted by $\zeta_{T}^{L_{\infty}}$, then it is taken as a change-point. We highlight that both $\zeta_{T}^{L_{2}}$ and $\zeta_{T}^{L_{\infty}}$ are of the form $C \sqrt{\log T}$, where $C$ is a positive constant, with its choice discussed in Section 3.1. In the Gaussian case, $\sqrt{\log T}$ is the order of the threshold used in order to prove consistency (with respect to the estimated number and location of the change-points) results; more information can be found in Anastasiou \& Fryzlewicz (2021). In this paper, our interest is in the actual numerical value of the threshold rather than in the theoretical order of its magnitude. Therefore, from now on, the $\sqrt{\log T}$ order is used, but it is calibrated through the constant, in order to optimise the performance of CCID in the specific applied framework described in this paper, which, is not of asymptotic interest.

A key ingredient of our CCID method is that, due to the aggregation of the (scaled) CUSUM statistics, the algorithm automatically identifies common change-points, rather than estimating single change-points at different locations in different components of the time series. This characteristic removes the need for post-processing across the $d$-dimensional sequence. 


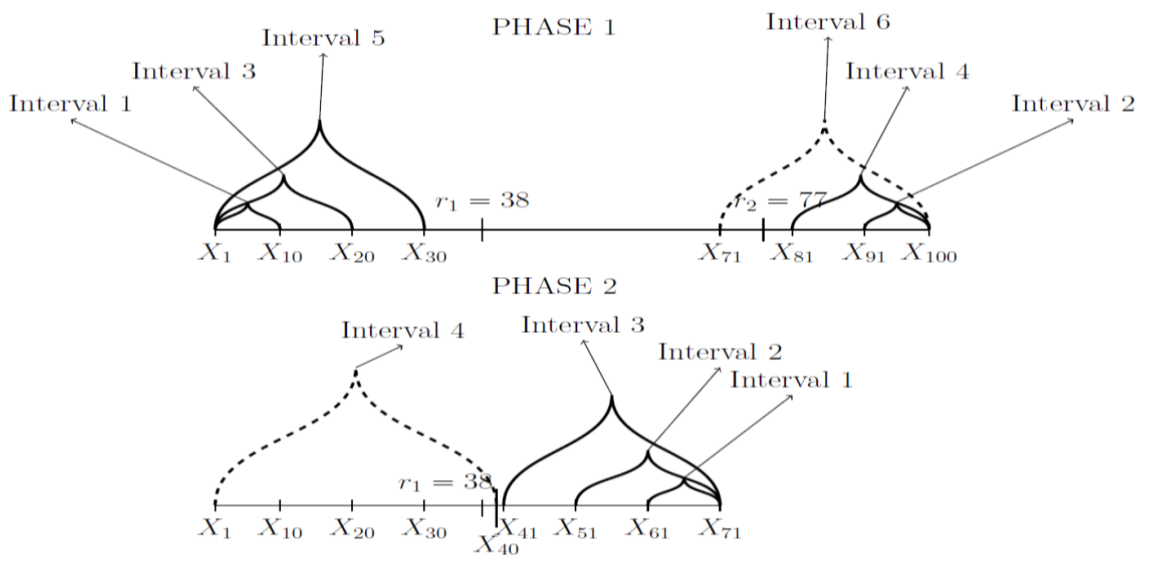

Figure 1: An example of how the segmentation method in CCID works. (Anastasiou \& Fryzlewicz, 2021)

\subsection{Matching the change-points to the relevant time series}

We now explain how CCID can provide information on which brain region data sequence(s) each changepoint arises from. The procedure to achieve this, is divided into two steps: firstly, estimate the change-points using CCID explained in Section 2.5. and secondly follow a post-processing approach, which is applied to each one of the $d$ univariate data sequences in order to match the detected change-points (obtained from the first step) with the relevant data sequences. We highlight that the post-processing carried out in this section is completely different to the postprocessing carried out in Section 2.5 above. Specifically, we begin by estimating the number and the location of the change-points using either the $L_{2}$ or the $L_{\infty}$ approach. The estimated change-points are then sorted in an increasing order in the set

$$
\hat{R}=\left\{\hat{r}_{1}, \hat{r}_{2}, \ldots, \hat{r}_{\hat{N}}\right\}
$$

where $\hat{N}$ is the number of the estimated change-points. For $\hat{r}_{0}=0, \hat{r}_{\hat{N}+1}=T$, and $\tilde{Y}_{s, e}^{b,(k)}$ in 8 , we then proceed, for each univariate data sequence $Y_{t, T}^{(k)}$ as in (7), with the calculation of the quantities

$$
\tilde{Y}_{\hat{r}_{j-1}+1, \hat{r}_{j+1}}^{\hat{r}_{j},(k)}, \quad \forall j \in\{1,2, \ldots, \hat{N}\}, \quad \forall k \in\{1,2, \ldots, d\}
$$

as defined in (8). Let us now denote by $S^{(k)}$ the set of estimated change-points that appear in the $k^{\text {th }}$ data sequence; as a note, keep in mind that, for any $k \in\{1,2, \ldots, d\}, S^{(k)}$ is a subset of $\hat{R}$ in (11). Since we now work in a univariate setting, checking all data sequences independently in order to match them with the obtained change-points, the values obtained in 12 are compared to a threshold value $\zeta_{T}^{*}$. If, for example, $\tilde{Y}_{\hat{r}_{j-1}+1, \hat{r}_{j+1}}^{\hat{r}_{j},(k)}>\zeta_{T}^{*}$, then it is deduced that the detected change-point $\hat{r}_{j}$ appears in the $k^{t h}$ data sequence. The threshold in this univariate setting is taken to be equal to $C \sqrt{\log T}$. At the beginning of our approach 
the sets $S^{(k)}, k=1,2, \ldots, d$ are empty. For each $k \in\{1,2, \ldots, d\}$, if $\tilde{Y}_{\hat{r}_{j-1}+1, \hat{r}_{j+1}}^{\hat{r}_{j},(k)}>\zeta_{T}^{*}$, then the estimated change-point $\hat{r}_{j}$ is added to the set $S^{(k)}$. We complete this for all $\hat{r}_{j}$ and for all the $d$ data sequences, so that we operate on the same set of change-points, $\hat{R}$, for each data sequence. Hence, the collection $\left\{S^{(k)}\right\}_{k=1,2, \ldots, d}$ provides the information on which cross periodogram (and hence the pairwise relationships between brain regions at given scales of resolution) each change-point arises from. Our R package ccid also has a function that associates the detected change-points with the respective data sequence (or sequences) from which it was detected, but only for the threshold approach.

We now provide more details on the threshold constant $C$ used in the post-processing procedure described in the previous paragraph. The threshold $\zeta_{T}^{*}$ is of the same order as the threshold used for univariate changepoint detection in a series of papers, such as Anastasiou \& Fryzlewicz (2021) and Fryzlewicz (2014). We note that $C=\sqrt{2}$ has been used in the aforementioned papers. More specifically, in Anastasiou \& Fryzlewicz (2021) it was proved that for i.i.d. Gaussian noise and while $T \rightarrow \infty$, the probability of a false detection when the null hypothesis of no change-point is satisfied goes to zero when $C=\sqrt{3}$. This means that, for our setting, it is sensible to take $C \leq \sqrt{3}$. In finite sample size simulations, Fang et al. 2020) showed that for the method in Fryzlewicz (2014), under the Gaussian i.i.d. setting, when the sample size is equal to $T=500$ and the threshold $\zeta_{T}^{*}=4.62$ (which in our setting means $C \approx 1.853$ ), then the results are very good only under the null model of no change-points, where the false positive rate is approximately 0.05. In the cases where there exist three, five, and eight change-points then the correct detection percentage with the aforementioned threshold is $82 \%, 64.3 \%$, and $34.4 \%$, respectively, which for the last two cases is especially low, meaning that a lower threshold is more suitable for data sets with multiple change-points. For CCID, we take $C=1.05 \times \sqrt{2}$, which is slightly higher than the proposed value of $\sqrt{2}$ in Anastasiou \& Fryzlewicz (2021) and Fryzlewicz (2014), while still lower than the aforementioned upper bound value of $\sqrt{3}$. We note that this threshold is used as a post-processing tool when change-points have already been detected by CCID. Therefore, we are more interested in maintaining a good detection power when we have multiple change-points rather than having a low false positive rate in the case of no change-point; the latter is already controlled in the main algorithm.

\subsection{The model selection approach}

An alternative approach to the $L_{2}$ and $L_{\infty}$ threshold methods is based on the optimization of a model selection criterion. In this setup, CCID begins by (hopefully) overestimating the number of change-points by choosing a suboptimal (lower) threshold value in the $L_{2}$ or the $L_{\infty}$ methods explained in Section 2.5 The estimated change-points are sorted in an increasing order in the set $\tilde{S}=\left\{\tilde{r}_{1}, \tilde{r}_{2}, \ldots, \tilde{r}_{\tilde{N}}\right\}$, for $\tilde{N} \geq N$ (the estimated number of change-points is greater than the true number of change-points). The next step is to run a change-point removal process using a joint approach for the $d$ data sequences. For $\tilde{r}_{0}=0$ and 
$\tilde{r}_{\tilde{N}+1}=T$, we collect the triplets $\left(\tilde{r}_{j-1}, \tilde{r}_{j}, \tilde{r}_{j+1}\right)$ and calculate

$$
C S^{*}\left(\tilde{r}_{j}\right):=\max _{k=1,2, \ldots, d}\left\{\tilde{Y}_{\tilde{r}_{j-1}+1, \tilde{r}_{j+1}}^{\tilde{r}_{j},(k)}\right\}
$$

We then define $m=\operatorname{argmin}_{j}\left\{C S^{*}\left(\tilde{r}_{j}\right)\right\}$, meaning that the estimation $\tilde{r}_{m}$ is the detected change-point which has the lowest (scaled) CUSUM statistic value in $[13) ; \tilde{r}_{m}$ is labelled the least important detection in the set $\tilde{S}$. This change-point is removed from the set, reducing $\tilde{N}$ by 1 . We relabel the remaining estimates (in increasing order) in $\tilde{S}$, and repeat this estimate removal process until $\tilde{S}$ becomes the empty set. After this, the vector

$$
\boldsymbol{b}=\left(b_{1}, b_{2}, \ldots, b_{\tilde{N}}\right)
$$

represents the collection of estimates where $b_{\tilde{N}}$ is the estimate that was first removed from $\tilde{S}, b_{\tilde{N}-1}$ is the estimate that was removed next, and so on. The vector $\boldsymbol{b}$ is called the solution path and we define the collection of models $\left\{\mathcal{M}_{j}\right\}_{j=0,1, \ldots, \tilde{N}}$ where $\mathcal{M}_{0}=\emptyset$ and $\mathcal{M}_{j}=\left\{b_{1}, b_{2}, \ldots, b_{j}\right\}$.

A key element in the construction of the information criterion is the likelihood function for our data. Due to the unknown dependence structure in our data, we work instead on an approximation of the likelihood, where the data are taken to be independent (see Sections 4.1 and 5.1 for a discussion on deviations from independence); this, from now on, is called the pseudo-likelihood, denoted by $\tilde{L}(\cdot ; \cdot)$, with its logarithm being $\tilde{\ell}(\cdot ; \cdot)$. For fixed $k \in\{1,2, \ldots, d\}$, if there are no change-points in the data sequence $\left\{Y_{t}^{(k)}\right\}_{t=1,2, \ldots, T}$ and $Y_{1}^{(k)}, Y_{2}^{(k)}, \ldots, Y_{T}^{(k)}$ are independent random variables, then as $Y_{t}^{(k)}$ follows the scaled $\chi_{1}^{2}$ distribution with mean $\sigma^{(k)}$, we have that

$$
\tilde{L}\left(\sigma^{(k)} ; \boldsymbol{y}^{(\boldsymbol{k})}\right)=\prod_{t=1}^{T} f\left(y_{t}^{(k)} \mid \sigma^{(k)}\right)=\prod_{t=1}^{T} \frac{\exp \left\{-\frac{y_{t}^{(k)}}{2 \sigma^{(k)}}\right\}}{\sqrt{2 \pi \sigma^{(k)} y_{t}^{(k)}}}
$$

where $y_{t}^{(k)}$ is the given $k^{t h}$ data sequence. Therefore,

$$
\begin{aligned}
\tilde{\ell}\left(\sigma^{(k)} ; \boldsymbol{y}^{(k)}\right) & =\sum_{t=1}^{T}\left\{-\frac{1}{2} \log 2 \pi \sigma^{(k)}-\frac{1}{2} \log y_{t}^{(k)}-\frac{y_{t}^{(k)}}{2 \sigma^{(k)}}\right\} \\
& =-\frac{T}{2} \log 2 \pi \sigma^{(k)}-\frac{1}{2} \sum_{t=1}^{T} \log y_{t}^{(k)}-\frac{1}{2 \sigma^{(k)}} \sum_{t=1}^{T} y_{t}^{(k)} .
\end{aligned}
$$

Using the maximum likelihood estimation method, based on 16 , the estimator for the parameter $\sigma^{(k)}$ is

$$
\hat{\sigma}^{(k)}=\frac{1}{T} \sum_{t=1}^{T} Y_{t}^{(k)} .
$$


In the situation that we have $J$ change-points at locations $r_{1}, r_{2}, \ldots, r_{J}$, then using (16) and (17), the pseudo-log-likelihood for the multivariate data sequence becomes

$$
R_{j}(\boldsymbol{\sigma} ; \boldsymbol{y})=\sum_{k=1}^{d} \sum_{j=1}^{J+1} \tilde{\ell}_{j}\left(\sigma^{j,(k)} ; \boldsymbol{y}^{(\boldsymbol{k})}\right)
$$

where $\sigma^{j,(k)}$ is the mean of the $k^{t h}$ data sequence in the segment $\left[r_{j-1}+1, r_{j}\right]$ and $\tilde{\ell}_{j}\left(\sigma^{j,(k)} ; \boldsymbol{y}^{(\boldsymbol{k})}\right)$ is the pseudo-log-likelihood in $\left[16\right.$ for the aforementioned segment, where $r_{0}=0$ and $r_{J+1}=T$. Therefore, from (16), we have that

$$
R_{j}(\boldsymbol{\sigma} ; \boldsymbol{y})=\sum_{k=1}^{d} \sum_{j=1}^{J+1}\left\{-\frac{r_{j}-r_{j-1}}{2} \log 2 \pi \sigma^{j,(k)}-\frac{1}{2} \sum_{t=r_{j-1}+1}^{r_{j}} \log y_{t}^{(k)}-\frac{1}{2 \sigma^{j,(k)}} \sum_{t=r_{j-1}+1}^{r_{j}} y_{t}^{(k)}\right\}
$$

Among the collection of models $\left\{\mathcal{M}_{j}\right\}_{j=0,1, \ldots, \tilde{N}}$, we propose to select the model that minimizes the following selection criterion

$$
\mathrm{IC}(j)=-R_{j}\left(\hat{\boldsymbol{\sigma}}_{\boldsymbol{j}} ; \boldsymbol{y}\right)+p_{T}=\frac{1}{2} \sum_{k=1}^{d}\left\{\sum_{t=1}^{T}\left(\log \left(2 \pi \hat{\sigma}_{t, j}^{(k)} y_{t}^{(k)}\right)\right)+\sum_{t=1}^{T} \frac{y_{t}^{(k)}}{\hat{\sigma}_{t, j}^{(k)}}\right\}+p_{T}
$$

where $p_{T}$ is an appropriately chosen penalty function which goes to infinity with $T$. For the definition of $\hat{\sigma}_{t, j}^{(k)}$, we denote for $j=2, \ldots, \tilde{N}$

$$
m_{1}^{(j)}<\ldots<m_{j}^{(j)}
$$

to be the sorted elements of $\mathcal{M}_{j}=\left\{b_{1}, b_{2}, \ldots, b_{j}\right\}$. For example if $j=2$ and $b_{2}<b_{1}$, we have that $m_{1}^{(2)}=b_{2}$ and $m_{2}^{(2)}=b_{1}$; where $b_{1}$ and $b_{2}$ are the first two elements of the solution path as in (14). Then, for $i=0,1, \ldots, j$

$$
\hat{\sigma}_{t, j}^{(k)}=\frac{1}{m_{i+1}^{(j)}-m_{i}^{(j)}} \sum_{t=m_{i}^{(j)}+1}^{m_{i+1}^{(j)}} y_{t}^{(k)}, \quad m_{i}^{(j)}+1 \leq t \leq m_{i+1}^{(j)},
$$

for $m_{i}^{(j)}, i \in\{1,2, \ldots, j\}$ as in 21] and $m_{0}^{(j)}=0, m_{j+1}^{(j)}=T$; basically $\hat{\sigma}_{t, j}^{(k)}, t=1,2, \ldots, T$ is the estimated piecewise-constant signal for the $k^{t h}$ data sequence when the detected change-points are $b_{1}, b_{2}, \ldots, b_{j}$. For the choice of $p_{T}$, see Section 3 .

\subsection{Estimating brain networks}

One of the main advantages of CCID over existing change-point methods is that CCID can detect changepoints that are close to one another. In some cases, the number of change-points itself could be the objective of the study. However, in other cases, researchers often would like to estimate a partition-specific brain network or the undirected graph between each pair of detected change-points. This helps visualize the FC 
network in a more precise fashion. Estimating an undirected graph for a partition with a small number of data points and a large number of brain regions $(p>>T)$ is difficult, especially in the case when $\delta$ (the minimum distance between change-points) is small (see Tables 9 and 10 in the Appendix). Nonetheless, CCID can be provided with a minimum distance between change-points as an input and it can postprocess the change-points to fit this criterion. If we let the full experimental time course be divided into subintervals separated by the detected change-points $\hat{r}_{1}, \hat{r}_{2}, \ldots, \hat{r}_{\hat{N}}$ then for each subinterval $\left[\hat{r}_{j}, \hat{r}_{j+1}\right)$, we can then estimate a sparse precision matrix to represent the FC brain network (Cribben \& Fiecas, 2016) for each partition using the following log-likelihood with a SCAD penalty (Fan \& Li, 2001) on the elements of the precision matrix (inverse covariance matrix)

$$
\max _{\Omega} \log \operatorname{det} \Omega-\operatorname{tr}(\mathbf{S} \Omega)-\sum_{i=1}^{P} \sum_{j=1}^{P} \operatorname{SCAD}_{\rho, a}\left(\left|\omega_{i j}\right|\right),
$$

where $\Omega$ is the precision matrix, $S$ is the sample covariance matrix, $\rho_{i j}(=\rho$ for convenience) is the penalty function on the elements of $\Omega, \omega_{i j}$, and $w_{i j}$ is the adaptive weight function. Fan \& Li (2001) defined the adaptive weights to be

$$
w_{i j}=1 /\left|\widetilde{\omega}_{i j}\right|^{\gamma}
$$

for tuning parameter $\gamma>0$, where $\widetilde{\omega}_{i j}$ is the $(i, j)$ th entry for any consistent estimate $\widetilde{\Omega}=\left(\widetilde{\omega}_{i j}\right)_{1 \leq i, j \leq P}$. Mathematically, the SCAD penalty is symmetric and a quadratic spline on $[0, \infty)$, whose first order derivative is

$$
\operatorname{SCAD}_{\rho, a}^{\prime}(x)=\rho\left(I(|x| \leq \rho)+\frac{(a \rho-|x|)_{+}}{(a-1) \rho} I(|x|>\rho)\right),
$$

for $x \geq 0$, where $I$ is an indicator function, with $\rho>0$ and $a>2$ being two tuning parameters. Zhu \& Cribben (2018) discuss the optimal choice for the tuning parameters in SCAD, and find that the best method for estimating sparse brain networks is SCAD in combination with the Bayesian Information Criterion.

\section{Data}

\subsection{Simulation study setup}

In this section we examine the performance of CCID through various simulations. For each simulation setting, we perform 100 repetitions, provide a diagram to illustrate how the second order structure (or FC network structure) changes over time and a quantified description of the results.

For CCID, we provide results for both the $L_{2}$ and the $L_{\infty}$ approaches as described in Section 2.5 and the results for the information criterion discussed in Section 2.7. For the threshold, we chose the constant $c_{1}=0.65$ for the $L_{2}$ approach and the constant $c_{2}=2.25$ for the $L_{\infty}$ approach as they provided a balance between specificity and sensitivity in all signals examined in a large scale simulation study; we highlight that 
the results presented in this paper are only a fraction of the various signals used in order to determine the value of the constants $c_{1}$ and $c_{2}$. It is important that CCID remains robust to alternative choices to this parameter and the practitioner has the option to obtain more change-points by decreasing this threshold value. We only present the results for the finest scale of -1 used in the wavelet transformation as fMRI data typically only have lag 1 autocorrelation (Fiecas et al. 2017 ). With regards to the information criterion model selection method, we define the penalty function by

$$
p_{T}=\frac{1}{2}\left(n_{j}\right) d(\log T)^{\alpha}
$$

where $n_{j}$ is the total number of estimated parameters related to the model $M_{j}, d(=p(p+1) / 2)$ is the dimensionality of the wavelet-transformed data sequences (the periodograms/cross periodograms), $T$ is the number of time points in each data sequence, and $\alpha>0$. We assume that $n_{j}$ manages to capture the complexity of the model under investigation. In (26), we propose a penalty function that is a function of the dimension of the periodograms and cross-periodograms, $d$. However, we should highlight that Zou et al. (2014) introduced a form of the Schwarz's Information Criterion similar to 20 and they used a penalty of the form $p_{T}=\frac{1}{2}\left(n_{j}\right)(\log T)^{2+\epsilon}$, where $\epsilon>0$, and in practice $\epsilon=0.1$. Through the empirical work in this paper, where the length, $T$, of the data sequences is not larger than 1000 observations, we find that the penalty suggested by Zou et al. (2014) gives rise to too many false positive change-point detections. This is expected because the penalty used in Zou et al. (2014) does not depend on the dimensionality $d$ of the time series, which can be quite large. A comparison of the two penalties is given through simulated data and it is available from Github at https://github.com/anastasiou-andreas/ccid. For both the simulated and fMRI data sets results that follow, we choose $\alpha=0.1$, which has been selected by considering many types of simulations involving a wide range of signals. The different structures used, consisted of general changes in the correlation/network structure, changes in the clustering (or community structure) and changes in the degree of a network, as well as no changes at all.

We compare CCID to three other competitor methods: Sparsified Binary Segmentation (SBS: Cho \& Fryzlewicz, 2015) which is available through the hdbinseg R package, Factor (Barigozzi et al., 2018) which is available through the factorcpt $\mathrm{R}$ package, and the method developed in Barnett \& Onnela (2016), which we denote by BO. There is no software available for BO, which is also the case for the majority of the methods mentioned in the introduction. However, for the BO approach, code was provided to us by the authors, which we had to adjust through a function that has already been written for the simulations carried out in Ofori-Boateng et al. (2020). For SBS, we only present the results for the finer scale of -1 used in the wavelet transformation. The Factor approach performs multiple change-point detection under factor modelling. The change-points are in the second order structure of both the common and idiosyncratic components. The main function in the $\mathrm{R}$ package factorcpt returns change-point estimates from the common and idiosyncratic 
components separately, and in our results we present both. The notation used for these is Factor com and Factor $i d$, respectively.

\subsection{Description of the settings}

We now describe the simulations. The objective of the simulation study is to mimic the properties of fMRI data under various settings. While the data are simulated using various models, we display the dependence between the time series using an undirected graph (or network, which we use interchangeably) structure. A graph consists of a set of vertices $V$ and corresponding edges $E$ that connect pairs of vertices. Here each vertex represents a time series, or ROI, and edges encode dependencies. In the fMRI setting, a missing edge indicates a lack of functional connectivity between corresponding regions. A graph of $\mathbf{X}$ can alternatively be represented using the precision matrix (inverse covariance matrix) of $\mathbf{X}$, with the elements of the matrix corresponding to edge weights. Here a missing edge between two vertices in the graph indicates conditional independence between the variables, giving rise to a zero element in the precision matrix.

- In Simulation 1, there are no true change-points. The data are simulated from a vector autoregression (VAR) model with $T=300$ and $p=15$. A $\operatorname{VAR}(1)$, a vector autoregression of order 1 , is given by $\mathbf{X}_{t}=\Pi \mathbf{X}_{t-1}+\epsilon_{t}, t=2, \ldots, T$, where $\Pi$ is an $(p \times p)$ coeffcient matrix and $\epsilon_{t}$ is an unobservable mean zero white noise vector process with time invariant covariance matrix. The VAR model is used to reconstruct the linear interdependency element prevalent among multivariate time series applications such as fMRI data (Cribben et al., 2012, Xu et al. 2020). For a visual display of the network structure, see Figure 24.

- In Simulation 2, we consider a change in the network edge degree with $T=200$ and $p=40$. The change-point occurs in the middle of the time series with the edge degree changing from $\eta=0.95$ to $\eta=0.05$ before and after the change-point, respectively, where $\eta$ represents the probability of a non-zero connection. The non-zero edges do not have the same strength. For a visual display of the change in the network structure, see Figure $2 \mathrm{~B}$. The simulation may appear quite easy (moving from a very dense graph to a very sparse graph), however, as the strength of the connections in both partitions are very small $(\leq 0.1)$ the simulation in fact is more difficult than it appears.

- In Simulation 3, the data are simulated from a VAR model with $T=500$ and $p=15$. There are four true change-points and they are located at equal intervals across the whole time line. For a visual display of the changes in the network structure, see Figure 3 A.

- In Simulation 4, the data are simulated from a VAR model with $T=600$ and $p=10$. There are seven true change-points and they are located at equal intervals across the whole time line. For a visual display of the changes in the network structure, see Figure 4 A. 

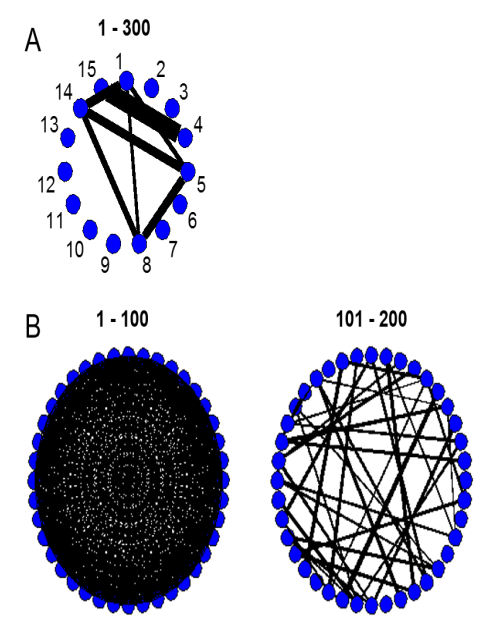

Figure 2: (A) The network structure for the VAR data set with no change-points (Simulation 1) and (B) The network structure for the change in the network degree with one change-point (Simulation 2). The ROI time series are represented by nodes, black edges infer positive connectivity, and the strength of connection between the ROIs is directly related to the thickness of the edges, that is, the thicker the edge the stronger the connection.
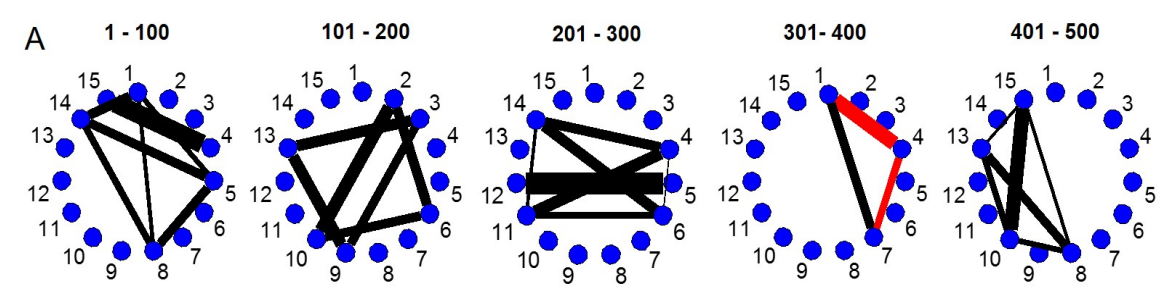

\section{8}
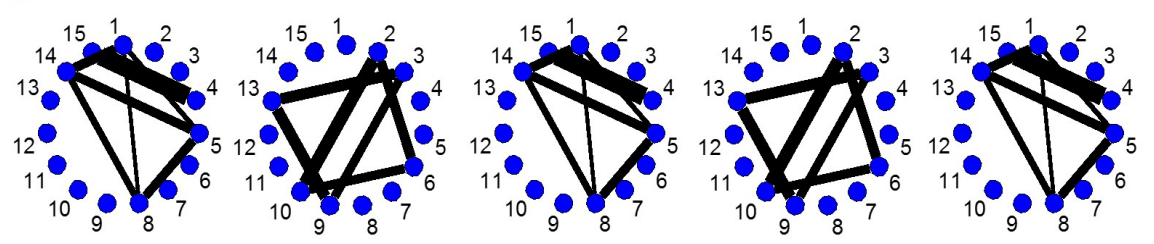

Figure 3: (A) The network structure for the VAR data set with four change-points (Simulation 3) and (B) The network structure for the VAR data set with four change-points (Simulation 5). The ROI time series are represented by nodes, black edges infer positive connectivity, and the strength of connection between the regions is directly related to the thickness of the edges, that is, the thicker the edge the stronger the connection.

- In Simulation 5, the data are simulated from a VAR model with $T=500$ and $p=15$. There are four true change-points and they are located at equal intervals across the whole time line. This simulation has an ABABA structure. For a visual display of the changes in the network structure, see Figure $3 \mathrm{~B}$.

- In Simulation 6, there are seven true change-points and they are located at equal intervals across the whole time line. The data are simulated from a VAR model with $T=600$ and $p=10$. This simulation has an ABABABAB structure. For a visual display of the changes in the network structure, 
see Figure $4 \mathrm{~B}$.
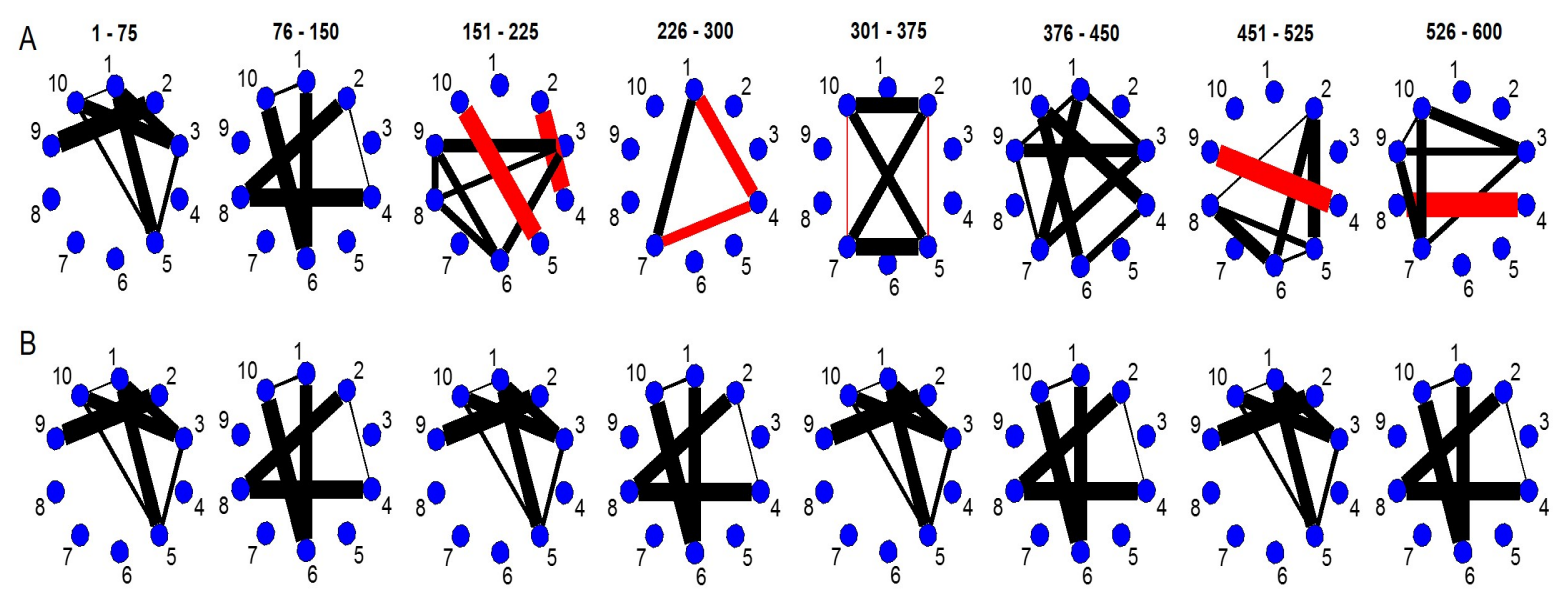

Figure 4: (A) The network structure for the VAR data set with seven change-points (Simulation 4) and (B) The network structure for the VAR data set with seven change-points (Simulation 6). The ROI time series are represented by nodes, black (red) edges infer positive (negative) connectivity, and the strength of connection between the regions is directly related to the thickness of the edges, that is, the thicker the edge the stronger the connection.

- In Simulation 7, we again consider a change in the network edge degree, however, in this simulation, there are seven true change-points. The change-points are located at equal intervals across the whole time line with $T=600$ and $p=40$, and the edge degree changing from $\eta=0.95$ to $\eta=0.05$ before and after the change-point. This simulation has an ABABABAB structure. For a visual display of the change in the network structure, see Figure 5 A.

- In Simulation 8, we consider a change in the network clustering (or community structure). There are again 7 change-points and they are located at equal intervals across the whole time line with $T=600$ and $p=30$. In the even time segments, the true number of communities $K_{o}=2$, that is, there are two clusters, with the within cluster correlation equal to 0.8 and the between cluster correlation equal to 0 . In the odd time segments, the true number of communities $K_{o}=6$ with the within cluster correlation equal to 0.75 and the between cluster correlation equal to 0.2 (Cribben \& Yu, 2017). This simulation has an ABABABAB structure. For a visual display of the change in the network structure, see Figure $5 \mathrm{~B}$.

- In Simulation 9, we consider a change in the network clustering (or community structure). It has exactly the same setup as Simulation 8 except the change-points are irregularly spaced with change points occurring at time points $100,175,275,300,400,475,575$.

- In Simulation 10, we consider a change in the network clustering (or community structure). There are 3 change-points and they are located at time points $100,175,275$, with $T=300$ and $p=100$. In the 
bioRxiv preprint doi: https://doi.org/10.1101/2020.12.20.423696; this version posted June 8, 2021. The copyright holder for this preprint (which was not certified by peer review) is the author/funder, who has granted bioRxiv a license to display the preprint in perpetuity. It is made available under aCC-BY-NC-ND 4.0 International license.

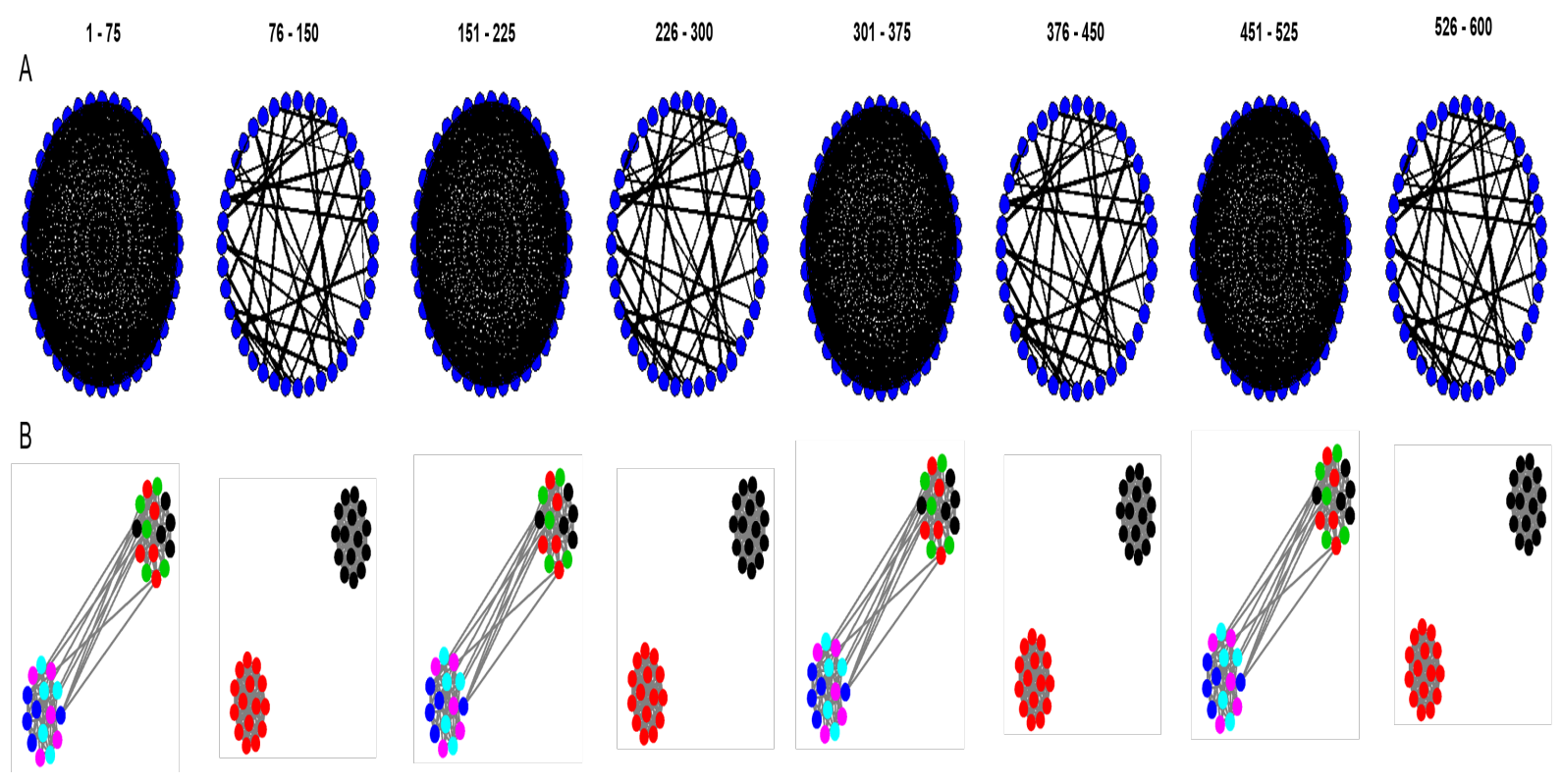

Figure 5: (A) The change in degree data set with seven change-points (Simulation 7). The ROI time series are represented by nodes, black edges infer positive connectivity, and the strength of connection between the regions is directly related to the thickness of the edges, that is, the thicker the edge the stronger the connection. (B) The change in clustering network structure with seven change-points (Simulation 8). In the even time segments, the true number of clusters is $K_{o}=2$ (cluster membership is based on node color) within the within cluster correlation equal to 0.8 and the between cluster correlation equal to 0 . In the odd time segments, the true number of clusters is $K_{o}=6$ (cluster membership is based on node color) within the within cluster correlation equal to 0.75 and the between cluster correlation equal to 0.2 .

$1-100$

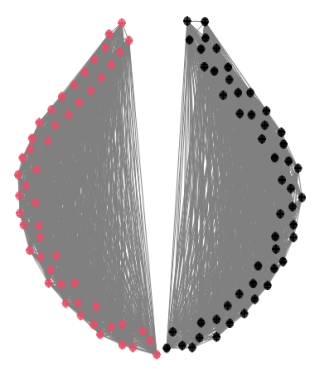

$101-175$

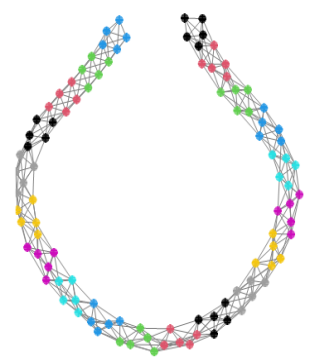

$176-275$

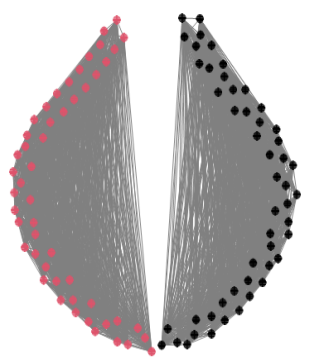

$276-300$

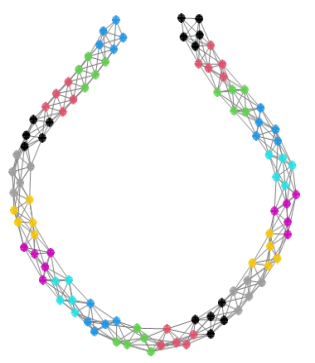

Figure 6: The change in clustering network structure with three change-points (Simulation 10). In the even time segments, the true number of clusters is $K_{o}=20$ (cluster membership is based on node color) with the within cluster correlation equal to 0.8 and the between cluster correlation equal to 0 . In the odd time segments, the true number of clusters is $K_{o}=2$ (cluster membership is based on node color) with the within cluster correlation equal to 0.75 and the between cluster correlation equal to 0.2 .

even time segments, the true number of communities $K_{o}=2$, that is, there are two clusters, with the within cluster correlation equal to 0.8 and the between cluster correlation equal to 0 . In the odd time segments, the true number of communities $K_{o}=20$ with the within cluster correlation equal to 0.75 
and the between cluster correlation equal to 0.2 (Cribben \& Yu, 2017). This simulation has an ABAB structure and is a high dimension data example. For a visual display of the change in the network structure, see Figure6.

Simulation 1 is similar to a steady state fMRI time series, where the network structure does not change over time. Simulations $5-10$ cover the situation where the subject alternates between 2 states (ABABA, ABABABAB and ABAB structures) and are similar to tasked based fMRI data sets. We hypothesize that our new method CCID will perform particularly well in these scenarios. This is due to CCID's ability to isolate the change-points between tasks within subintervals and then to detect them with those subintervals. This is in contrast to the popular binary segmentation (BS) method which has been the only method applied on fMRI data. BS searches the entire time course for one change-point. Once a change-point is found, the data is split into two subsegments (hence, the term binary). A similar search is performed on each subsegment, possibly giving rise to further change-points. BS is a greedy algorithm as it is performed sequentially with each stage depending on the previous ones, which are never re-visited. Hence, BS looks to partition the experimental time course into two intervals which is difficult given the similarity between any two intervals for this particular data type. In other words, for any time points in an ABABA experimental structure, the before and after structures are very similar to the average of the A and B network structures, thus making change-points difficult to identify. However, CCID has the ability to find multiple change-points that are very close to one another.

\subsection{Accuracy metrics}

In the simulations, we compare the accuracy of the different methods using the frequency distribution of $\hat{N}-N$, that is, the difference between the number of detected change-points and the number of true change-points. If $\hat{N}-N>0$, it indicates that the method finds too many false positive change-points, while if $\hat{N}-N<0$, the method does not find all the true change-points. $\hat{N}-N=0$ is ideal.

As a measure of the accuracy of the detected locations in time of the detected change-points compared to the location of the true change-points, we also provide the scaled Hausdorff distance,

$$
d_{H}=n_{s}^{-1} \max \left\{\max _{j} \min _{k}\left|r_{j}-\hat{r}_{k}\right|, \max _{k} \min _{j}\left|r_{j}-\hat{r}_{k}\right|\right\}
$$

where $n_{s}$ is the length of the largest segment, $\hat{r}_{k}$ are the estimated change-points and $r_{j}$ are the true changepoints. The optimal model obtains a minimum scaled Hausdorff distance. The average computational time for all methods is also provided. 


\subsection{Task based fMRI data set}

The data was taken from an anxiety-inducing experiment (Wager et al. 2009). The task was a variant of a well-studied laboratory paradigm for eliciting social threat, in which participants must give a speech under evaluative pressure. The design was an off-on-off design, with an anxiety-provoking speech preparation task occurring between lower anxiety resting periods. Participants were informed that they were to be given $2 \mathrm{~min}$ to prepare a 7 min speech, and that the topic would be revealed to them during scanning. They were told that after the scanning session they would deliver the speech to a panel of expert judges, though there was "small chance" they would be randomly selected not to give the speech. After the start of fMRI acquisition, participants viewed a fixation cross for $2 \mathrm{~min}$ (resting baseline). At the end of this period, participants viewed an instruction slide for $15 \mathrm{~s}$ that described the speech topic, which was "why you are a good friend". The slide instructed participants to be sure to prepare enough for the entire 7 min period. After 2 min of silent preparation, another instruction screen appeared (a relief instruction, $15 \mathrm{~s}$ duration) that informed participants that they would not have to give the speech. An additional 2 min period of resting baseline completed the functional run.

Social threat is a cause of mental stress that generates physiological responses in the body (Rozanski et al. 1988). However, the connection between the human brain processes and the peripheral physiology while under stress of social threat is poorly understood. The objective of the study was to assess functional connectivity elicited by public speech preparation (SET) and its relationship with heart rate. Previous human neuroimaging studies (Critchley et al., 2000, Gianaros et al. 2004) indicated that the most likely areas for brain generators of cardiovascaular and other peripheral physiology responses to social evaluative threat are in the medial prefrontal cortex (MPFC), which projects to a set of cortical regions including the striatum. However, given MPFC's heterogeneity, it may contain subregions with diverse relationships with regulation. In addition, animal studies implicated the ventromedial prefrontal cortex (Amat et al., 2005. 2008).

Functional blood-oxygen-level-dependent (BOLD) images were acquired with a $\mathrm{T}^{*}$-sensitive spiral in-out sequence $\left(\mathrm{TR}\right.$ (repetition time) $=2000 \mathrm{~ms}$, TE (echo time) $=40 \mathrm{~ms}$, flip angle $=90^{\circ}, 24$ slices in ascending sequential sequence, $4.5 \times 3.4375 \times 3.475 \mathrm{~mm}$ voxels). An LCD projector displayed stimuli on a backprojection screen placed in the scanner room. Functional images were subjected to a standard preprocessing sequence. Slice-timing acquisition correction using sync interpolation was performed using custom software, and realignment of the functional images to correct for head movement was performed using the Automated Image Registration tools (Woods et al. 1998). For details on the remaining preprocessing steps see Wager et al. (2009).

During the course of the experiment a series of 215 images were acquired ( $\mathrm{TR}=2 \mathrm{~s}$ ). In order to create ROIs, the voxel time series were averaged across the entire regions. The data consists of 4 ROIs and heart 
rate for $n=23$ subjects. The temporal resolution of the heart rate was $1 \mathrm{~s}$ compared to $2 \mathrm{~s}$ for the fMRI data. Hence, the heart rate was down-sampled by taking every other measurement.

\subsection{Resting state fMRI data set}

The second data set is a resting-state fMRI data set, as described in Habeck et al. (2012); Cribben \& $\mathrm{Yu}$ (2017). Participants $(n=45)$ are instructed to rest in the scanner for 9.5 minutes, with the instruction to keep their eyes open for the duration of the scan. Functional images were acquired using a 3.0 Tesla magnetic resonance scanner (Philips) using a field echo-planar imaging (FE-EPI) sequence $[\mathrm{TE} / \mathrm{TR}=20$ $\mathrm{ms} / 2000 \mathrm{~ms}$; flip angle $=72^{\circ} ; 112 \times 112$ matrix; in-plane voxel size $=2.0 \mathrm{~mm} \times 2.0 \mathrm{~mm}$; slice thickness $=3.0$ mm (no gap); 37 transverse slices per volume]. In addition, a T1-weighted turbo field echo high resolution image was also acquired $\left[\mathrm{TE} / \mathrm{TR}=2.98 \mathrm{~ms} / 6.57 \mathrm{~ms}\right.$; flip angle $=8^{\circ} ; 256 \times 256$ matrix; in-plane voxel size $=1.0 \mathrm{~mm} \times 1.0 \mathrm{~mm}$; slice thickness $=1.0 \mathrm{~mm}$ (no gap); 165 slices]. The individual time series data were bandpass-filtered between 0.009 and $0.08 \mathrm{~Hz}$, motion corrected and co-registered to the structural data, with a subsequent spatial normalization to the MNI template. The voxel time courses at white-matter and CSF locations are submitted to a Principal Component Analysis and, together with the motion parameters, we use all components with an eigenvalue strictly greater than 1 as independent variables in a subsequent nuisance regression Habeck et al. (2012). Each voxel's time series is residualized with respect to those independent variables. The residual time series images are then smoothed with an isotropic Gaussian kernel (FWHM = $6 \mathrm{~mm}$ ). We apply the Anatomical Automatic Labeling (Tzourio-Mazoyer et al., 2002) atlas to the adjusted voxel-wise time series and produce time series for 31 Regions of Interest (ROIs) for each subject by averaging the voxel time series within the ROIs. The 31 ROIs contain 8 regions from the attentional network (frontal superior medial L, angular L, angular R, temporal middle L, temporal mid R, thalamus L, cerebellum crus1 L, cerebellum crus1 R), 2 regions from the visual network (temporal superior L, temporal superior R), 3 regions from the sensorimotor network (postcentral L, postcentral R, supplementary motor area R), 7 regions the salience network (cingulum anterior L, frontal mid L, frontal middle R, insula L, insula R, supramarginal L, supramarginal R ), 9 regions from the default mode network (precentral L, precentral R, parietal superior $\mathrm{L}$, occipital superior R, parietal inferior L, parietal inferior R, temporal inferior L, temporal inferior R, cingulum posterior L) and 2 regions from the auditory network (calcarine L, Calcarine R). We chose these networks because an increasing number of pathologic conditions appear to be reflected in the functional connectivity between these particular brain regions and we wanted the number of ROIs in the fMRI data to match the simulation settings. In total, each ROI time series is made up of 285 time points (9.5 minutes with $\mathrm{TR}=2$ ). 


\section{Results}

\subsection{Simulation study}

In this section, we present the simulation and fMRI results. Table 1 shows the results over 100 iterations for Simulation 1 (no change-point: see Section 3.2). SBS (Section 3.1), Factor (Section 3.1), and our method, CCID (across both thresholds and information criteria), perform very well and correctly do not detect changepoints across almost all iterations. BO has the worst performance in terms of Type I error. CCID is at least an order of magnitude computationally faster than all competing methods, a pattern which is also evident for the rest of the simulations.

Table 1: The distribution of $\hat{N}-N$ over 100 simulated data sequences from Simulation 1 (no change-point) for Sparsified Binary Segmentation (SBS), Factor with common components (Factor com), Factor with idiosyncratic components (Factor id), Barnett \& Onnela (2016) (BO), and Cross-covariance isolate detect with the $L_{2}$ threshold (CCID. $L_{2}$ ), the $L_{\infty}$ threshold (CCID. $L_{\infty}$ ), the $L_{2}$ information criterion (CCID. $\left.L_{2} I C\right)$ and the $L_{\infty}$ information criterion (CCID. $L_{\infty} I C$ ). The computational times for each method are also provided.

\begin{tabular}{|c|c|c|c|c|c|}
\hline \multirow[b]{2}{*}{ Method } & \multicolumn{4}{|c|}{$\hat{N}-N$} & \multirow[b]{2}{*}{ Time (s) } \\
\hline & 0 & 1 & 2 & $\geq 3$ & \\
\hline SBS & 97 & 3 & 0 & 0 & 2.65 \\
\hline Factor com & 100 & 0 & 0 & 0 & 14.16 \\
\hline Factor id & 95 & 5 & 0 & 0 & 14.16 \\
\hline $\mathrm{BO}$ & 83 & 14 & 3 & 0 & 13.97 \\
\hline CCID. $L_{2}$ & 92 & 6 & 2 & 0 & 0.25 \\
\hline CCID. $L_{\infty}$ & 90 & 9 & 1 & 0 & 0.27 \\
\hline CCID. $L_{2} I C$ & 100 & 0 & 0 & 0 & 0.36 \\
\hline CCID. $L_{\infty} I C$ & 100 & 0 & 0 & 0 & 0.29 \\
\hline
\end{tabular}

Table 2 shows the results over 100 iterations for Simulation 2 (change in network degree, see Section 3.2 ). As mentioned previously, this simulation is more difficult than it appears. SBS, Factor with idiosyncratic components (Factor id), BO and CCID (except for CCID. $L_{\infty}$ ) perform very well and correctly identify the change-point in the vast majority of the 100 iterations, with Factor id performing the best, finding one change point in all 100 iterations. On the other hand, Factor with common components (Factor com) performs poorly and does not detect the change-point in any of the 100 iterations. In terms of Hausdorff distance, Factor id performs the best but there is a negligible difference with SBS, BO, CCID. $L_{2}$, CCID. $L_{2} I C$ and CCID. $L_{\infty} I C$. 
Table 2: The distribution of $\hat{N}-N$ and the scaled Hausdorff distance over 100 simulated data sequences from Simulation 2 (change in network degree) for Sparsified Binary Segmentation (SBS), Factor with common components (Factor com), Factor with idiosyncratic components (Factor id), Barnett \& Onnela (2016) (BO), and Cross-covariance isolate detect with the $L_{2}$ threshold (CCID. $L_{2}$ ), the $L_{\infty}$ threshold (CCID. $L_{\infty}$ ), the $L_{2}$ information criterion (CCID. $L_{2} I C$ ) and the $L_{\infty}$ information criterion (CCID. $L_{\infty} I C$ ). The computational times for each method are also provided.

\begin{tabular}{|c|c|c|c|c|c|c|}
\hline \multirow[b]{2}{*}{ Method } & \multicolumn{4}{|c|}{$\hat{N}-N$} & \multirow[b]{2}{*}{$d_{H}$} & \multirow[b]{2}{*}{ Time (s) } \\
\hline & -1 & 0 & 1 & $\geq 2$ & & \\
\hline SBS & 0 & 99 & 1 & 0 & 0.02 & 11.20 \\
\hline Factor com & 100 & 0 & 0 & 0 & 1 & 15.39 \\
\hline Factor id & 0 & 100 & 0 & 0 & 0.01 & 15.39 \\
\hline $\mathrm{BO}$ & 0 & 93 & 7 & 0 & 0.05 & 7.78 \\
\hline CCID. $L_{2}$ & 5 & 93 & 0 & 2 & 0.06 & 1.00 \\
\hline CCID. $L_{\infty}$ & 0 & 57 & 24 & 14 & 0.26 & 1.10 \\
\hline CCID. $L_{2} I C$ & 3 & 93 & 4 & 0 & 0.07 & 1.62 \\
\hline CCID. $L_{\infty} I C$ & 3 & 94 & 3 & 0 & 0.06 & 2.41 \\
\hline
\end{tabular}

For Simulation 3 (see Section 3.2), SBS, BO and CCID (using the thresholding and information criteria) perform well with the majority of their detected change-points within \pm 2 (Table 3 ). However, CCID. $L_{2} I C$ performs the best in terms of the distribution of $\hat{N}-N$, that is, the majority of its detected change-points equal the true number of change-points across all 100 iterations, but BO has a slightly lower Hausdorff distance, the detected change-points are closest to the true change-points in terms of location. Both Factor com and Factor id perform poorly in this simulation.

Table 3: The distribution of $\hat{N}-N$ and the scaled Hausdorff distance over 100 simulated data sequences from Simulation 3 for Sparsified Binary Segmentation (SBS), Factor with common components (Factor com), Factor with idiosyncratic components (Factor id), Barnett \& Onnela (2016) (BO), and Cross-covariance isolate detect with the $L_{2}$ threshold (CCID. $\left.L_{2}\right)$, the $L_{\infty}$ threshold (CCID. $L_{\infty}$ ), the $L_{2}$ information criterion (CCID. $L_{2} I C$ ) and the $L_{\infty}$ information criterion (CCID. $\left.L_{\infty} I C\right)$. The computational times for each method are also provided.

\begin{tabular}{|l|l|l|l|l|l|l|l|l|l|}
\hline & \multicolumn{7}{|c|}{$\hat{N}-N$} & & \\
Method & $\leq-3$ & -2 & -1 & 0 & 1 & 2 & $\geq 3$ & $d_{H}$ & Time (s) \\
\hline SBS & 4 & 6 & 18 & 72 & 0 & 0 & 0 & 0.51 & 5.93 \\
Factor com & 100 & 0 & 0 & 0 & 0 & 0 & 0 & 4 & 15.38 \\
Factor id & 74 & 7 & 9 & 10 & 0 & 0 & 0 & 2.73 & 15.38 \\
BO & 0 & 0 & 0 & 72 & 22 & 6 & 0 & 0.22 & 45.45 \\
CCID. $L_{2}$ & 0 & 2 & 37 & 57 & 4 & 0 & 0 & 0.46 & 0.42 \\
CCID. $L_{\infty}$ & 0 & 3 & 13 & 71 & 10 & 3 & 1 & 0.36 & 0.46 \\
CCID. $L_{2} I C$ & 1 & 2 & 10 & 80 & 6 & 1 & 0 & 0.27 & 0.82 \\
CCID. $L_{\infty} I C$ & 9 & 7 & 7 & 72 & 5 & 0 & 0 & 0.66 & 0.63 \\
\hline
\end{tabular}

Simulation 4 (see Section 3.2 contains 7 change-points with CCID (across both thresholds and information criteria) outperforming SBS and Factor in terms of detection and having the smallest Hausdorff distances (Table 4). However, BO also performs well and has the third best Hausdorff distance after CCID. $L_{2}$ and CCID. $L_{2} I C$. CCID. $L_{2} I C$ performs the best while SBS is able to detect some change points, however, Factor performs poorly in this simulation with large negative values for $\hat{N}-N$ and large Hausdorff distance values. $\mathrm{BO}$ is particularly slow in this simulation. 
Table 4: The distribution of $\hat{N}-N$ and the scaled Hausdorff distance over 100 simulated data sequences from Simulation 4 for Sparsified Binary Segmentation (SBS), Factor with common components (Factor com), Factor with idiosyncratic components (Factor id), Barnett \& Onnela (2016) (BO), and Cross-covariance isolate detect with the $L_{2}$ threshold (CCID. $L_{2}$ ), the $L_{\infty}$ threshold (CCID. $L_{\infty}$ ), the $L_{2}$ information criterion (CCID. $L_{2} I C$ ) and the $L_{\infty}$ information criterion (CCID. $\left.L_{\infty} I C\right)$. The computational times for each method are also provided.

\begin{tabular}{|l|l|l|l|l|l|l|l|l|l|}
\hline \multicolumn{1}{|c|}{} & \multicolumn{7}{|c|}{$\hat{N}-N$} & & \\
Method & $\leq-3$ & -2 & -1 & 0 & 1 & 2 & $\geq 3$ & $d_{H}$ & Time (s) \\
\hline SBS & 49 & 28 & 21 & 2 & 0 & 0 & 0 & 1.66 & 4.27 \\
Factor com & 100 & 0 & 0 & 0 & 0 & 0 & 0 & 7 & 16.12 \\
Factor id & 100 & 0 & 0 & 0 & 0 & 0 & 0 & 5.03 & 16.12 \\
BO & 0 & 0 & 1 & 43 & 34 & 16 & 6 & 0.30 & 95.80 \\
CCID. $L_{2}$ & 0 & 0 & 1 & 52 & 39 & 7 & 1 & 0.24 & 0.21 \\
CCID. $L_{\infty}$ & 12 & 10 & 27 & 36 & 13 & 1 & 1 & 0.94 & 0.27 \\
CCID. $L_{2} I C$ & 0 & 1 & 9 & 71 & 14 & 3 & 2 & 0.26 & 0.92 \\
CCID. $L_{\infty} I C$ & 4 & 14 & 23 & 54 & 5 & 0 & 0 & 0.65 & 0.47 \\
\hline
\end{tabular}

Simulations 1-4 show that CCID performs as well (and in most cases better than) the competing methods for the general settings. We now consider Simulations 5-8 which cover the situation where the subject changes between 2 states (ABABA and ABABABA structures) and mimic a tasked based fMRI experiment. This is a generalization of the epidemic change alternative (Kirch et al., 2015), change where change-point $r_{1}$ corresponds to the time when a process $\prod_{1}$ is turned off (equivalently, when another process $\prod_{2}$ is activated) and change-point $r_{2}$ corresponds to the time $\prod_{2}$ is turned off, thus returning to $\prod_{1}$. As we have stated before, we hypothesize that our new method, CCID, will perform particularly well in these scenarios. This is due to CCID's ability to isolate the change-points between tasks within subintervals and then to detect them with those subintervals. In addition, CCID has the ability to find change-points that are very close to one another.

Table 5 shows the results from Simulation 5 (see Section 3.2), which has four change-points and an ABABA network structure. Clearly CCID with both thresholds and information criteria outperform SBS and Factor com in terms of estimating the correct number of change points and the location of the detected change points. In particular, CCID. $L_{2}$ has the best performance, correctly identifying the correct number of change points in 91 out of 100 iterations and having the smallest Hausdorff distance. Furthermore, CCID. $L_{\infty}$ also performs well with similar but superior results to Factor id and BO. 
Table 5: The distribution of $\hat{N}-N$ and the scaled Hausdorff distance over 100 simulated data sequences from Simulation 5 for Sparsified Binary Segmentation (SBS), Factor with common components (Factor com), Factor with idiosyncratic components (Factor id), Barnett \& Onnela (2016) (BO), and Cross-covariance isolate detect with the $L_{2}$ threshold (CCID. $\left.L_{2}\right)$, the $L_{\infty}$ threshold (CCID. $L_{\infty}$ ), the $L_{2}$ information criterion (CCID. $L_{2} I C$ ) and the $L_{\infty}$ information criterion (CCID. $L_{\infty} I C$ ). The computational times for each method are also provided.

\begin{tabular}{|l|l|l|l|l|l|l|l|l|l|}
\hline & \multicolumn{7}{|c|}{$\hat{N}-N$} & & \\
Method & $\leq-3$ & -2 & -1 & 0 & 1 & 2 & $\geq 3$ & $d_{H}$ & Time (s) \\
\hline SBS & 71 & 26 & 1 & 2 & 0 & 0 & 0 & 3.24 & 4.45 \\
Factor com & 100 & 0 & 0 & 0 & 0 & 0 & 0 & 4 & 15.23 \\
Factor id & 7 & 17 & 0 & 76 & 0 & 0 & 0 & 0.63 & 15.23 \\
BO & 6 & 25 & 1 & 30 & 29 & 9 & 0 & 0.87 & 33.65 \\
CCID. $L_{2}$ & 0 & 1 & 4 & 91 & 4 & 0 & 0 & 0.13 & 0.44 \\
CCID. $L_{\infty}$ & 2 & 8 & 13 & 59 & 16 & 2 & 0 & 0.60 & 0.53 \\
CCID. $L_{2} I C$ & 24 & 15 & 0 & 61 & 0 & 0 & 0 & 1.24 & 0.81 \\
CCID. $L_{\infty} I C$ & 47 & 21 & 1 & 31 & 0 & 0 & 0 & 2.25 & 0.66 \\
\hline
\end{tabular}

Table 6 shows the results from Simulation 6 (see Section 3.2), which has seven network change points and an ABABABAB network structure. Again, CCID with both thresholds and information criteria outperforms all of SBS, Factor and BO. In particular, CCID. $L_{2}$ has the best performance in terms of estimating the correct number of change points and the location of the detected change points. SBS and Factor perform poorly in this simulation, they are unable to identify the correct number of change points in all iterations. BO is able to estimate the correct number of change points in 10 out of the 100 iterations but is very slow computationally compared to all the other methods.

Table 6: The distribution of $\hat{N}-N$ and the scaled Hausdorff distance over 100 simulated data sequences from Simulation 6 for Sparsified Binary Segmentation (SBS), Factor with common components (Factor com), Factor with idiosyncratic components (Factor id), Barnett \& Onnela (2016) (BO), and Cross-covariance isolate detect with the $L_{2}$ threshold (CCID. $\left.L_{2}\right)$, the $L_{\infty}$ threshold (CCID. $L_{\infty}$ ), the $L_{2}$ information criterion (CCID. $L_{2} I C$ ) and the $L_{\infty}$ information criterion (CCID. $\left.L_{\infty} I C\right)$. The computational times for each method are also provided.

\begin{tabular}{|l|l|l|l|l|l|l|l|l|l|}
\hline & \multicolumn{7}{|c|}{$\hat{N}-N$} & & \\
Method & $\leq-3$ & -2 & -1 & 0 & 1 & 2 & $\geq 3$ & $d_{H}$ & Time (s) \\
\hline SBS & 100 & 0 & 0 & 0 & 0 & 0 & 0 & 6.73 & 2.31 \\
Factor com & 100 & 0 & 0 & 0 & 0 & 0 & 0 & 7 & 17.14 \\
Factor id & 100 & 0 & 0 & 0 & 0 & 0 & 0 & 6.72 & 17.14 \\
BO & 47 & 13 & 4 & 10 & 13 & 8 & 5 & 2.38 & 53.33 \\
CCID. $L_{2}$ & 0 & 0 & 1 & 68 & 23 & 7 & 1 & 0.20 & 0.11 \\
CCID. $L_{\infty}$ & 40 & 26 & 13 & 17 & 4 & 0 & 0 & 1.86 & 0.17 \\
CCID. $L_{2} I C$ & 9 & 12 & 2 & 65 & 12 & 0 & 0 & 0.75 & 0.46 \\
CCID. $L_{\infty} I C$ & 34 & 26 & 5 & 33 & 2 & 0 & 0 & 2.28 & 0.25 \\
\hline
\end{tabular}

Table 7 shows the results from Simulation 7 (see Section 3.2, which has seven change points in the network degree ABABABAB type structure. Similar to Simulation 2, this simulation (see Figure 5A) may appear quite easy (alternating between a very dense graph to a very sparse graph), however, as the strength of the connections in both partitions is very small $(\leq 0.1)$ the simulation in fact is more difficult than it appears. Both SBS and Factor com struggle, they are unable to identify the correct number of change points 
in all iterations. CCID with both thresholds and information criteria outperforms SBS and Factor. BO, while superior to both SBS and Factor, is still inferior to CCID across both thresholds and information criteria. CCID. $L_{\infty} I C$ has the best performance in terms of estimating the correct number of change points and the location of the detected change points. In all 100 iterations, it identifies the correct number of true change points. However, Factor id performs adequately but in all iterations misses two change points.

Table 7: The distribution of $\hat{N}-N$ and the scaled Hausdorff distance over 100 simulated data sequences from Simulation 7 for Sparsified Binary Segmentation (SBS), Factor with common components (Factor com), Factor with idiosyncratic components (Factor id), Barnett \& Onnela (2016) (BO), and Cross-covariance isolate detect with the $L_{2}$ threshold (CCID. $\left.L_{2}\right)$, the $L_{\infty}$ threshold (CCID. $L_{\infty}$ ), the $L_{2}$ information criterion (CCID. $L_{2} I C$ ) and the $L_{\infty}$ information criterion (CCID. $\left.L_{\infty} I C\right)$. The computational times for each method are also provided.

\begin{tabular}{|l|l|l|l|l|l|l|l|l|l|}
\hline & \multicolumn{7}{|c|}{$\hat{N}-N$} & & \\
Method & $\leq-3$ & -2 & -1 & 0 & 1 & 2 & $\geq 3$ & $d_{H}$ & Time (s) \\
\hline SBS & 100 & 0 & 0 & 0 & 0 & 0 & 0 & 4.93 & 30.85 \\
Factor com & 100 & 0 & 0 & 0 & 0 & 0 & 0 & 7 & 67.02 \\
Factor id & 0 & 100 & 0 & 0 & 0 & 0 & 0 & 1.22 & 67.02 \\
BO & 11 & 5 & 0 & 73 & 10 & 1 & 0 & 0.62 & 115.49 \\
CCID. $L_{2}$ & 0 & 0 & 0 & 12 & 32 & 27 & 29 & 0.19 & 0.10 \\
CCID. $L_{\infty}$ & 0 & 0 & 0 & 0 & 6 & 8 & 86 & 0.14 & 0.12 \\
CCID. $L_{2} I C$ & 0 & 0 & 0 & 52 & 26 & 18 & 4 & 0.18 & 0.54 \\
CCID. $L_{\infty} I C$ & 0 & 0 & 0 & 100 & 0 & 0 & 0 & 0.03 & 0.34 \\
\hline
\end{tabular}

Table 8 shows the results from Simulation 8 (see Section 3.2), which has seven change points in the network clustering ABABABAB type structure. This is the most difficult simulation but, again, CCID with both thresholds and information criteria outperform SBS, Factor and BO. Similar to Simulation 7, CCID. $L_{\infty} I C$ has the best performance in terms of estimating the correct number of change points and the location of the detected change points. SBS and Factor perform poorly in this simulation, they are unable to identify the correct number of change points in all iterations. BO has a decent performance but is outperformed by CCID across all thresholds and information criteria.

Table 8: The distribution of $\hat{N}-N$ and the scaled Hausdorff distance over 100 simulated data sequences from Simulation 8 for Sparsified Binary Segmentation (SBS), Factor with common components (Factor com), Factor with idiosyncratic components (Factor id), Barnett \& Onnela (2016) (BO), and Cross-covariance isolate detect with the $L_{2}$ threshold (CCID. $\left.L_{2}\right)$, the $L_{\infty}$ threshold (CCID. $L_{\infty}$ ), the $L_{2}$ information criterion (CCID. $L_{2} I C$ ) and the $L_{\infty}$ information criterion (CCID. $L_{\infty} I C$ ). The computational times for each method are also provided.

\begin{tabular}{|l|l|l|l|l|l|l|l|l|l|}
\hline & \multicolumn{7}{|c|}{$\hat{N}-N$} & & \\
Method & $\leq-3$ & -2 & -1 & 0 & 1 & 2 & $\geq 3$ & $d_{H}$ & Time (s) \\
\hline SBS & 100 & 0 & 0 & 0 & 0 & 0 & 0 & 5.82 & 16.85 \\
Factor com & 100 & 0 & 0 & 0 & 0 & 0 & 0 & 7 & 423.40 \\
Factor id & 100 & 0 & 0 & 0 & 0 & 0 & 0 & 6.63 & 423.40 \\
BO & 39 & 5 & 1 & 29 & 22 & 4 & 0 & 2.11 & 82.27 \\
CCID. $L_{2}$ & 0 & 0 & 0 & 42 & 28 & 20 & 10 & 0.27 & 0.82 \\
CCID. $L_{\infty}$ & 0 & 0 & 0 & 2 & 6 & 13 & 79 & 0.30 & 1.26 \\
CCID. $L_{2} I C$ & 0 & 0 & 0 & 79 & 13 & 5 & 3 & 0.19 & 3.79 \\
CCID. $L_{\infty} I C$ & 0 & 0 & 1 & 94 & 4 & 1 & 0 & 0.11 & 3.12 \\
\hline
\end{tabular}


Table 9 shows the results from Simulation 9 (see Section 3.2), which has seven change points (ABABABAB) in the network clustering structure. It has the same setup as Simulation 8, but the true change-points are occurring at unequally spaced time points, namely at time points $t=100,175,275,300,400,475,575$. CCID with both thresholds and information criteria outperform SBS, Factor and BO, and to a larger extent than the results for Simulation 8. Again, similar to Simulation 8, the information criteria have the best performance in terms of estimating the correct number of change points and the location of the detected change points (smallest Hausdorff distance), with CCID. $\mathrm{L}_{\infty} \mathrm{IC}$ detecting the true number of change points on 89 out of 100 iterations. SBS and Factor perform poorly in this simulation, they are unable to identify the correct number of change points across all iterations. BO has a moderate performance but is outperformed by CCID across all thresholds and information criteria.

Table 9: The distribution of $\hat{N}-N$ and the scaled Hausdorff distance over 100 simulated data sequences from Simulation 9 for Sparsified Binary Segmentation (SBS), Factor with common components (Factor com), Factor with idiosyncratic components (Factor id), Barnett \& Onnela (2016) (BO), and Cross-covariance isolate detect with the $L_{2}$ threshold (CCID. $\left.L_{2}\right)$, the $L_{\infty}$ threshold (CCID. $L_{\infty}$ ), the $L_{2}$ information criterion (CCID. $L_{2} I C$ ) and the $L_{\infty}$ information criterion (CCID. $L_{\infty} I C$ ). The computational times for each method are also provided.

\begin{tabular}{|l|l|l|l|l|l|l|l|l|l|}
\hline & \multicolumn{7}{|c|}{$\hat{N}-N$} & & \\
Method & $\leq-3$ & -2 & -1 & 0 & 1 & 2 & $\geq 3$ & $d_{H}$ & Time (s) \\
\hline SBS & 100 & 0 & 0 & 0 & 0 & 0 & 0 & 5.21 & 14.11 \\
Factor com & 100 & 0 & 0 & 0 & 0 & 0 & 0 & 5.64 & 49.32 \\
Factor id & 100 & 0 & 0 & 0 & 0 & 0 & 0 & 5.75 & 49.32 \\
BO & 72 & 18 & 8 & 2 & 0 & 0 & 0 & 3.63 & 68.49 \\
CCID. $L_{2}$ & 0 & 0 & 0 & 16 & 24 & 25 & 45 & 0.36 & 2.01 \\
CCID. $L_{\infty}$ & 0 & 0 & 0 & 2 & 3 & 11 & 84 & 0.22 & 2.84 \\
CCID. $L_{2} I C$ & 1 & 12 & 0 & 63 & 13 & 9 & 2 & 0.29 & 5.48 \\
CCID. $L_{\infty} I C$ & 0 & 3 & 0 & 89 & 4 & 4 & 0 & 0.10 & 3.98 \\
\hline
\end{tabular}

Table 10 shows the results from Simulation 10 (see Section 3.2, which has three change points in the network clustering structure (ABAB). Here, we consider a high-dimensional time series $(p=100)$. CCID with both information criteria has the best performance, BO and CCID with both thresholds perform similarly and have the next best performance, with Factor having the worst performance (it is unable to identify the correct number of change points in all iterations). BO has a moderate performance but is outperformed by CCID with the information criteria. CCID. $\mathrm{L}_{\infty} \mathrm{IC}$ detects the true number of change points on 89 out of 100 iterations. The computation for CCID is an order of magnitude faster than SBS and two orders of magnitudes faster than $\mathrm{BO}$ and Factor. Finally, it is important to point out that the true dimensionality of the problem is $d(d+1) / 2$, where $d$ is the dimensionality of the initial multivariate time series. Hence, for given data of dimensionality $p=100$, the dimensionality of the problem is $d=5050$ (periodograms). 
Table 10: The distribution of $\hat{N}-N$ and the scaled Hausdorff distance over 100 simulated data sequences from Simulation 10 for Sparsified Binary Segmentation (SBS), Factor with common components (Factor com), Factor with idiosyncratic components (Factor id), Barnett \& Onnela (2016) (BO), and Cross-covariance isolate detect with the $L_{2}$ threshold (CCID. $\left.L_{2}\right)$, the $L_{\infty}$ threshold (CCID. $L_{\infty}$ ), the $L_{2}$ information criterion (CCID. $L_{2} I C$ ) and the $L_{\infty}$ information criterion (CCID. $L_{\infty} I C$ ). The computational times for each method are also provided.

\begin{tabular}{|l|l|l|l|l|l|l|l|l|l|}
\hline & \multicolumn{7}{|c|}{$\hat{N}-N$} & & \\
Method & -3 & -2 & -1 & 0 & 1 & 2 & $\geq 3$ & $d_{H}$ & Time (s) \\
\hline SBS & 0 & 0 & 95 & 5 & 0 & 0 & 0 & 1.01 & 81.91 \\
Factor com & 100 & 0 & 0 & 0 & 0 & 0 & 0 & 2.75 & 568.72 \\
Factor id & 5 & 0 & 95 & 0 & 0 & 0 & 0 & 1.08 & 568.72 \\
BO & 19 & 0 & 3 & 50 & 25 & 2 & 1 & 0.67 & 712.49 \\
CCID. $L_{2}$ & 0 & 0 & 0 & 36 & 21 & 20 & 23 & 0.28 & 0.97 \\
CCID. $L_{\infty}$ & 0 & 0 & 0 & 0 & 1 & 0 & 99 & 0.57 & 1.38 \\
CCID. $L_{2} I C$ & 0 & 0 & 0 & 73 & 9 & 8 & 10 & 0.15 & 3.86 \\
CCID. $L_{\infty} I C$ & 0 & 0 & 0 & 89 & 4 & 4 & 3 & 0.08 & 3.25 \\
\hline
\end{tabular}

As stated at the beginning of Section 2, CCID assumes that the ROI time series are Gaussian. It also assumes independence in the pseudo-likelihood as expressed in (15). However, CCID is robust under deviations from Gaussianity and/or from independence within the ROI time series. For example, Table 13 (in the Appendix) shows the results from applying CCID (and the competing methods, SBS, Factor and BO) to Simulations 1, 3 and 5, where the noise of each ROI time series now follows the $t$ distribution with 5 degrees of freedom. While the results are inferior to the Gaussian data, there is not a vast drop off in performance. The performance of CCID remains superior to SBS, Factor and BO in these settings. In situations where we suspect that our data are far from independent and Gaussian, our CCID algorithm can still be applied to the data after some pre-processing takes place. More specifically, towards this purpose, the subsampling and pre-averaging techniques described in Section 5.1 are beneficial.

In summary, for general data sets (Simulations 1-4), we found that CCID performs as well as, if not better than, SBS, Factor and BO. For simulations where the subject alternates between two states (Simulations 5-10), CCID performs very well and clearly outperforms SBS, Factor and BO using both thresholds and information criteria. Our recommendation is to use CCID with thresholding for general settings and to use the information criteria for the alternating structure, although both the thresholds and the information criteria outperform the competing methods in this case. With respect to the computational cost, our method (any variant of it) is computationally faster than any other competitor and this can be seen in the last column of the tables.

\subsection{Task based fMRI results}

We now present the results of our method, CCID, on the task based fMRI data set described in Section 3.4 the results for SBS, Factor and BO are in the Appendix. For this data set, we apply CCID to each subject separately, that is, we do not align the change-points in any way across the subjects. Figure 7 shows the detected change-points for the 4 ROI and heart rate data using CCID with threshold $L_{\infty}$ (top) and 

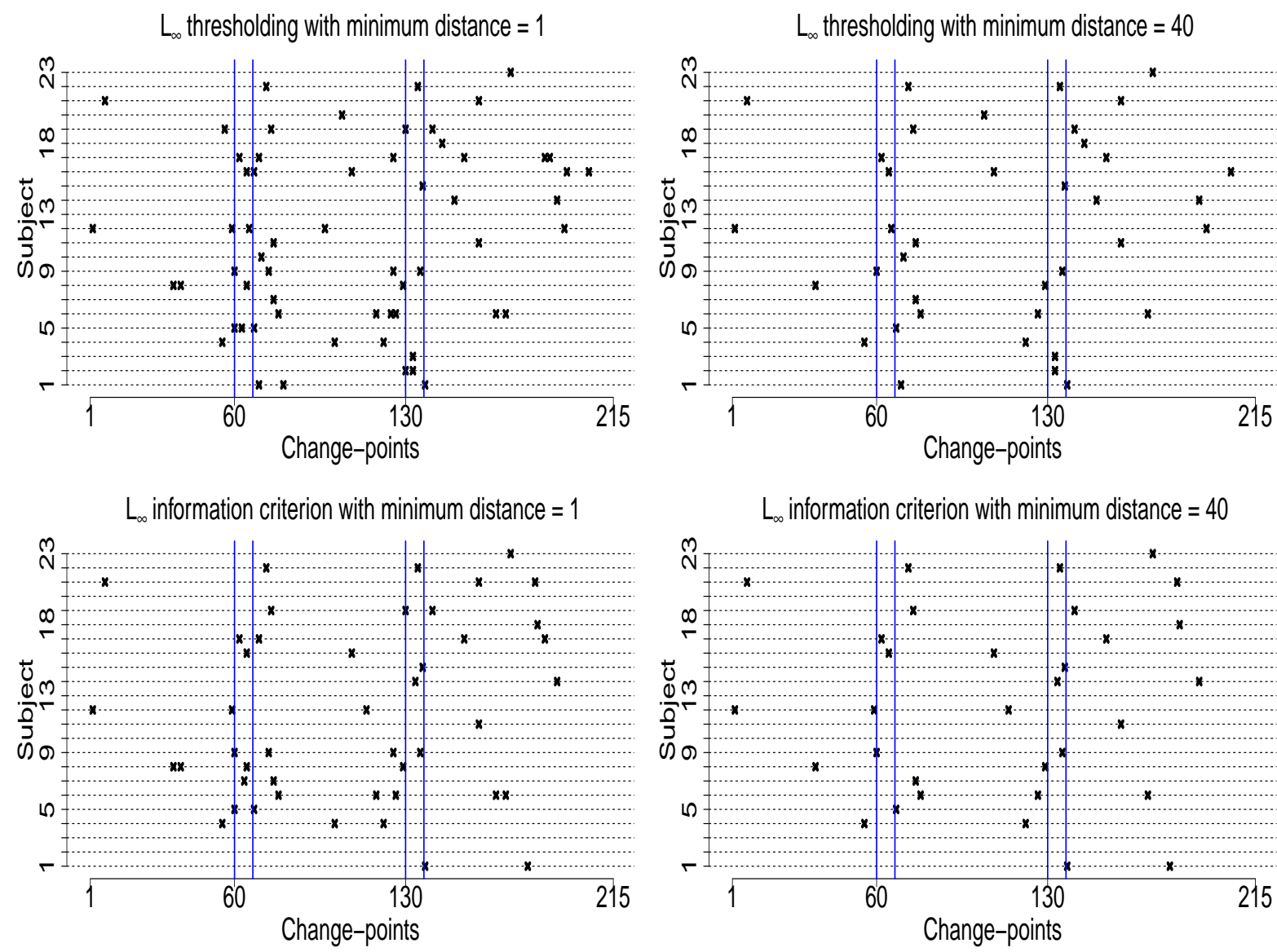

Figure 7: The detected change-points for the 4 ROI and heart rate data using CCID with threshold $L_{\infty}$ (top) and information criterion $L_{\infty} I C$ (bottom) using a minimum distance between change-points of $\delta=1$ (left) and $\delta=40$ (right). The $\mathrm{y}$-axis depicts the subject number while the $\mathrm{x}$-axis shows the change-point location times. The blue vertical lines indicate the times of the showing and of the removal of the visual cues.

information criterion $L_{\infty} I C$ (bottom) using a minimum distance between change-points of $\delta=1$ (left) and $\delta=40$ (right). The blue vertical lines indicate the times of the showing and of the removal of the visual cues. In some experiments, the number of change-points itself could be the objective of the study. Hence, a minimum distance $\delta=1$ could be utilized. However, in other cases, researchers often would like to estimate a partition specific brain network or the undirected graph between each pair of detected change-points. This helps visualize the FC network in a more precise fashion. To this end, we used a minimum distance $\delta=40$ (however, this is at the discretion of the researcher). For more details on the precise location of the change-points see Table 11 in the Appendix. The $\mathrm{y}$-axis depicts the subject number while the $\mathrm{x}$-axis shows the change-point location times. As we have already stated, one of the main advantages of CCID over previous change-point methods is that CCID can detect change-points that are very close to one another and is not limited by a minimum distance between change-points input. Hence, CCID is not only able to detect 
change-points that are in the neighborhood of time point 60 , which corresponds directly to the presentation of the first visual cue specifying the topic of the speech but also at the removal of said cue at time point 67.5 (see subjects 5, 12, 16 and 17 in the $L_{\infty}$ with minimum distance $=1$ in Figure 7 , top left). Likewise for time points 130 and 137.5, the second visual cue stating that the participants would in fact not have to give the presentation to the expert panel of judges after the conclusion of the scanning session was revealed (see subjects 2, 10, 19 in the $L_{\infty}$ with minimum distance $=1$ in Figure 7 , top left). The change-points occurring prior to the first visual cue may be related to anticipation of the speech topic, while change-points occurring during cues may be due to the different modes of anxiety as subjects silently prepare their speech. Finally, change-points occurring after the second visual cue may be due to the different modes of rest as subjects silently come to the end of the experiment. This pattern of change points is also seen for CCID with $L_{\infty}$ (Figure 7, bottom left). CCID is the only existing method (to the best of our knowledge) capable of finding change points this close to one another.

As mentioned above, often researchers would like to estimate a partition specific brain network or the undirected graph between each pair of detected change-points. This helps visualize the FC network in a more precise fashion. Hence, Figure 7 (right) shows the results where a minimum distance of $\delta=40$ was used. In this case, every subject has either two or three change-points with each subject having a change-point in the neighborhood of time point 60 (the first visual cue) and time point 130 (the second visual cue).

In addition, Figure 8 shows the detected change-points for the 4 ROI and heart rate data using CCID with threshold $L_{2}$ (top) and information criterion $L_{2} I C$ (bottom) using a minimum distance between changepoints of $\delta=1$ (left) and $\delta=40$ (right). All of the conclusions that we described above in Figure 7 are also true for these methods. The only difference is that CCID with threshold $L_{2}$ (top) and information criterion $L_{2} I C$ detect more change points for both $\delta=1,40$.

Figure 9 shows the density plots for the detected change-points in the 4 ROI and heart rate data using all variants of CCID across all 23 subjects using a minimum distance between change points of $\delta=40$. Again, the blue vertical lines indicate the times of the showing and of the removal of the visual cues. We can see that for all combinations of CCID there are clear peaks around time points 60-67.5 and 130-137.5, the times of the showing and the removal of the visual cues. There is consistency across the combinations although the thresholding provide the most distinct peaks.

For comparison, Figure 14 (in the Appendix) shows the detected change-points for the 4 ROI and heart rate data using the competing methods: SBS, Factor, and BO. Here, SBS and Factor idiosyncratic find at most one change-point for each subject and in the vast majority of cases they find no change-point at all. To be more precise, SBS does not find any change-points in 18 out of 23 subjects and Factor idiosyncratic in 16 subjects. None of the detected change-points by SBS are in the neighborhood of time point 60 , which corresponds directly to the presentation of the first visual cue specifying the topic of the speech and the 

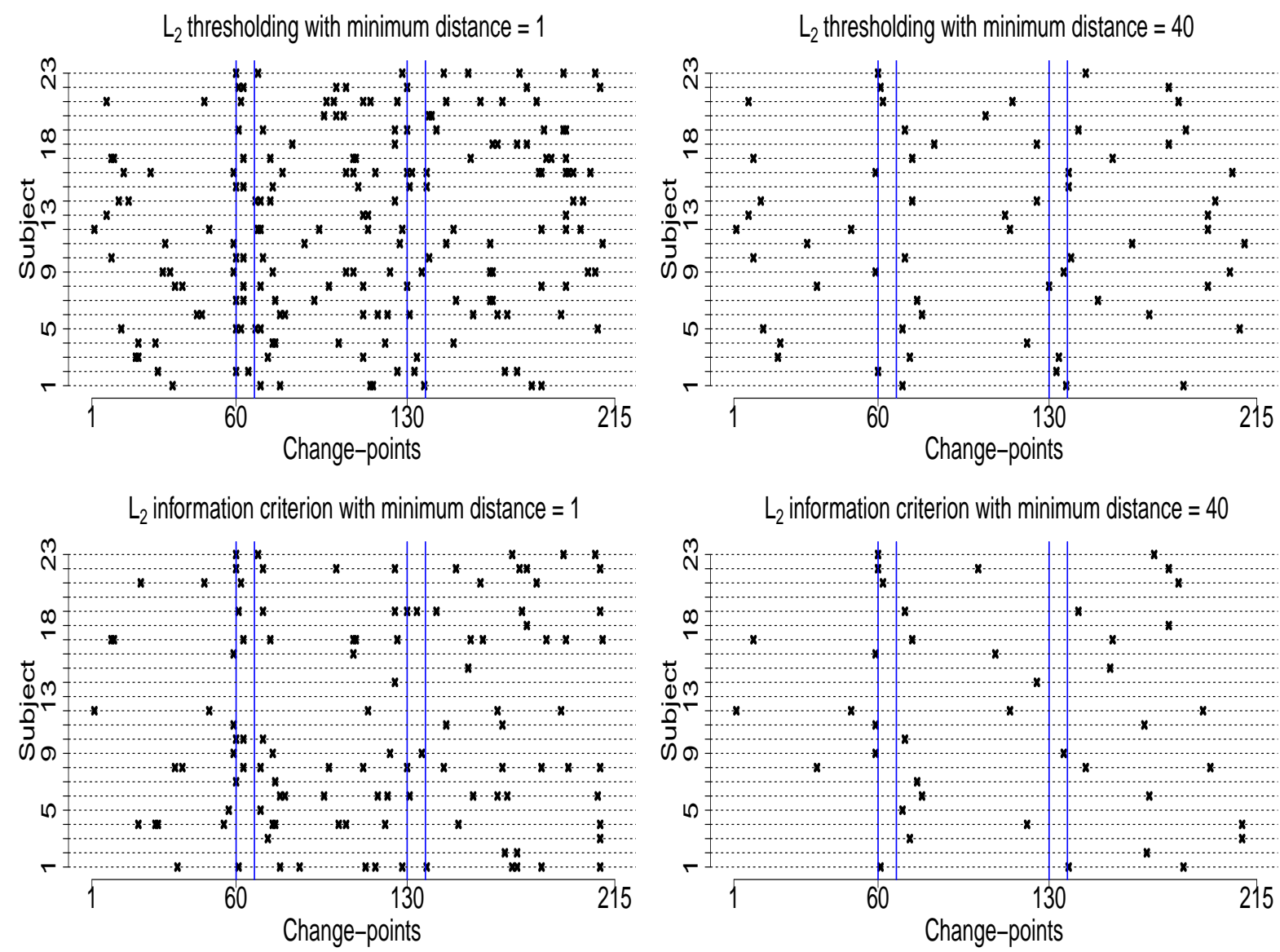

Figure 8: The detected change-points for the 4 ROI and heart rate data using CCID with threshold $L_{2}$ (top) and information criterion $L_{2} I C$ (bottom) using a minimum distance between change-points of $\delta=1$ (left) and $\delta=40$ (right). The y-axis depicts the subject number while the x-axis shows the change-point location times. The blue vertical lines indicate the times of the showing and of the removal of the visual cues.

removal of said cue at time point 67.5 (135 seconds). Only one change-point is near time point 130 (subject 15), the second visual cue stating that the participants would in fact not have to give the presentation to the expert panel of judges after the conclusion of the scanning session was revealed. The other change-points may be due to the different modes of anxiety as subjects silently prepare their speech. The BO method detects two change-points in 4 subjects, one change-point in 5 subjects and no change-points in 14 subjects. Factor common detects three change-points in 1 subject, two change-points in also 1 subject, one change-point in 6 subjects and no change-points in 15 out of 23 subjects.

As mentioned before we reduced the number of change-points that CCID detected by specifying a minimum distance between change points. There are two other procedures available to CCID to reduce the number of detected change-points. First, the thresholds in the $L_{\infty}$ and $L_{2}$ approaches (see Section 3.1 for a discussion) and the penalties for the $L_{\infty}$ and $L_{2}$ information criteria approaches can be increased. Second, 
Density plot for $\mathrm{L}_{2}$ thresholding with minimum distance $=40$

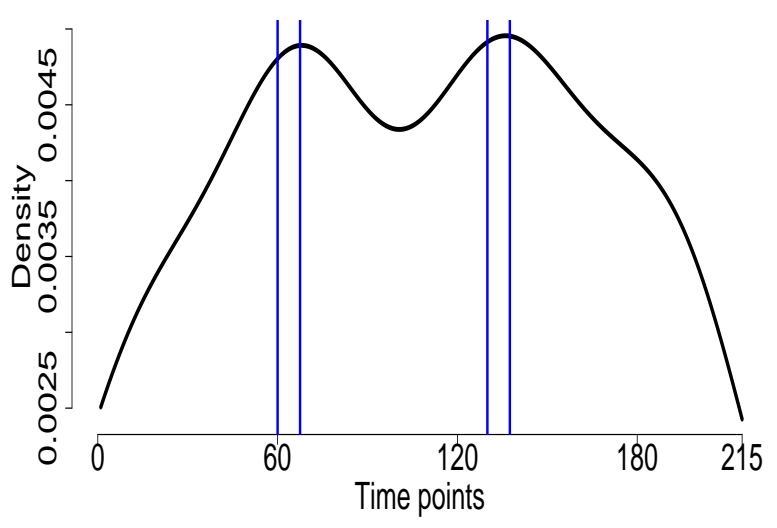

Density plot for $\mathrm{L}_{2} \mathrm{IC}$ with minimum distance $=40$

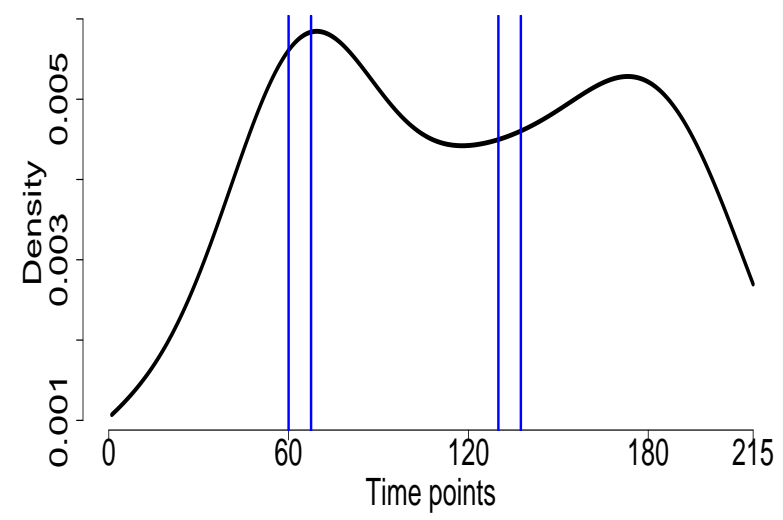

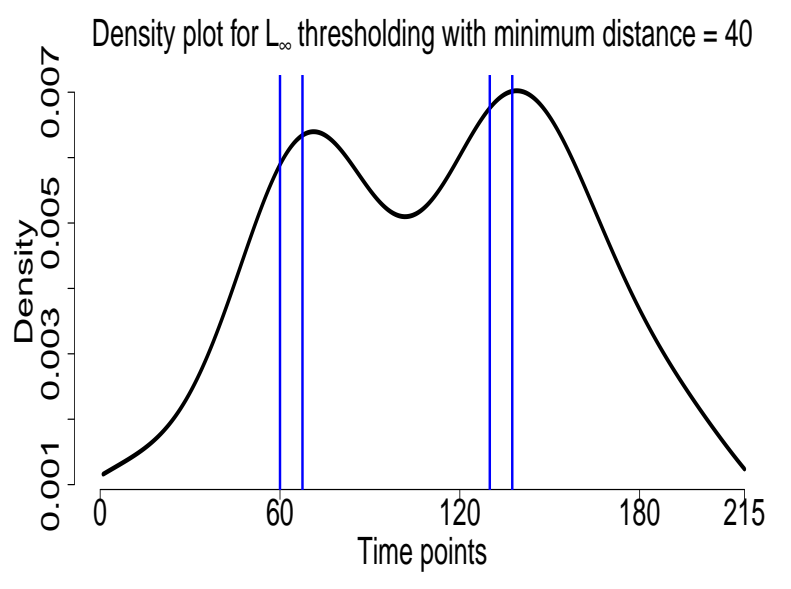

Density plot for $L_{\infty} I C$ with minimum distance $=40$

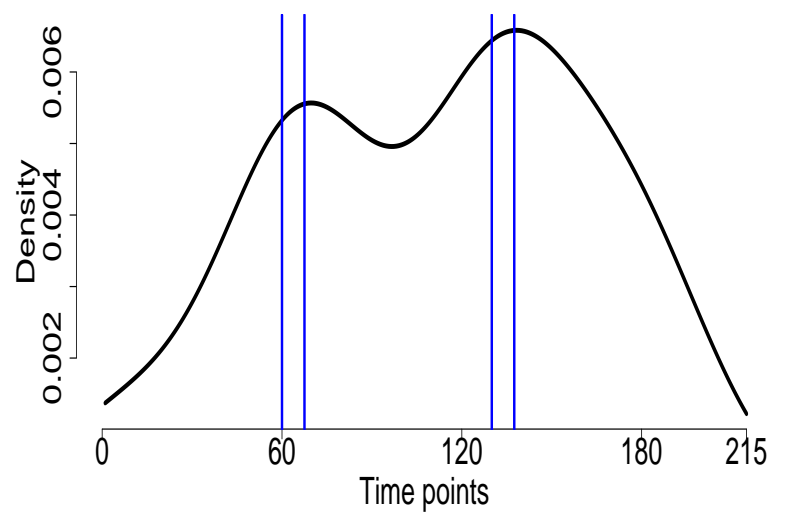

Figure 9: The density plots for the detected change-points in the 4 ROI and heart rate data using CCID across all 23 subjects using $\delta=40$. Top row: CCID with $L_{2}$ thresholding (left) and CCID with $L_{\infty}$ thresholding (right) Bottom row: CCID with $L_{2}$ Information-Criterion (left) and CCID with $L_{\infty}$ Information-Criterion (right).

CCID provides the solution path for the $L_{\infty}$ and $L_{2}$ thresholding approaches, which is an ordering of the importance of the detected change-points in descending order (see Section 2.6 for more details). Hence, a certain number of change-points could be specified using this procedure. Figure 10 shows the density plots for the detected change-points in the 4 ROI and heart rate data across all 23 subjects using a minimum distance between change points of $\delta=40$, using the solution path method for the $L_{\infty}$ and $L_{2}$ thresholding approaches, where we assume two change-points per channel. Both of these density plots show a good behavior with a clear bimodal structure that peak close to the first and second visual cues. The results from this procedure are comparable to the original robust choices we used in Figure 9. Of course, specifying a certain number of change-points would benefit from input from the domain knowledge experts. These procedures show the flexibility of CCID and allows the researchers alternative strategies to reduce the number of detected change-points.

The FC undirected graphs, estimated by SCAD and BIC (Section 2.8), for subjects 4, 6, 8, 9 and 11 

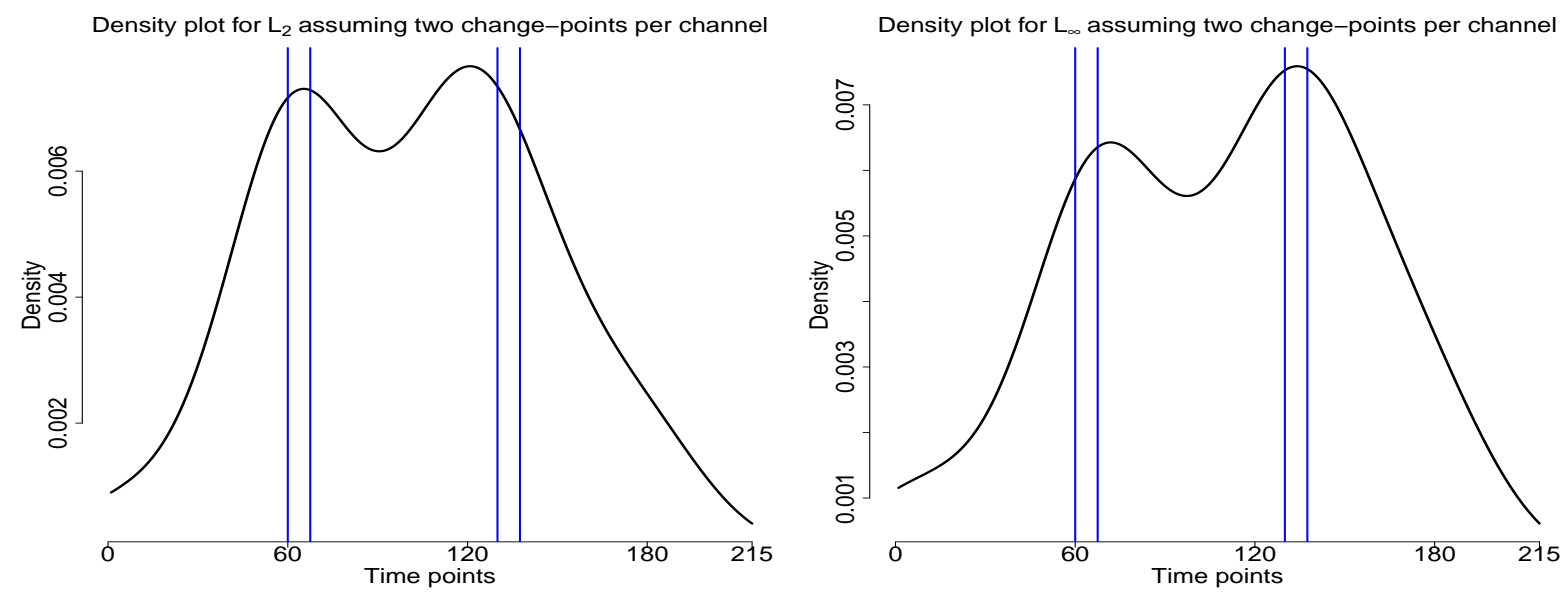

Figure 10: The density plots for the detected change-points in the 4 ROI and heart rate data using CCID across all 23 subjects using $\delta=40$ using the solution path method for the $L_{\infty}$ and $L_{2}$ thresholding approaches, where we assume two change-points per channel. CCID with $L_{2}$ thresholding (left) and CCID with $L_{\infty}$ thresholding (right)

between the detected change-points for CCID. $L_{\infty}$ are displayed in Figure 11. Black (red) edges infer positive (negative) connectivity, and the strength of connection between the regions is directly related to the thickness of the edges, that is, the thicker the edge the stronger the connection. The connectivity structure in the undirected graphs for each subject is quite different, however, the connection between the anterior mPFC and VMPFC is consistent across all subjects. From the graphs it is also evident that HR is more connected to the other ROIs during the resting periods, while during the second partition, when the speech topic is presented and the participants begin to silently prepare their speech, there is less connections between HR and the other ROIs. Perhaps this is due to the anticipation stage of the experiment being more stressful than the preparation stage.

\subsection{Resting state fMRI results}

Evidence of the non-stationary behavior of brain activity time series has been observed not only in task based fMRI studies, but also prominently in resting-state data ((Delamillieure et al., 2010, Doucet et al. 2012 Ondrus et al. 2021, Xiong \& Cribben, 2021). In these experiment mental activity is unconstrained. Figure 12 shows the detected change-points for the resting state fMRI data using CCID with threshold $L_{\infty}$ (top) and information criterion $L_{\infty} I C$ (bottom) using a minimum distance between change-points of $\delta=1$ (left) and $\delta=40$ (right). For more details on the precise location of the change-points see Table 12 in the Appendix. The $\mathrm{y}$-axis depicts the subject number while the $\mathrm{x}$-axis shows the change-point location times. Where the minimum distance between change-points is not restricted, every subject has at least three change-points in their second order structure structure with the maximum number of change-points being 30 (subject 1). The results indicate that, not only does the number of second-order structure change-points differ across subjects, but the location of the change-points is also variable. In addition, some subjects remain 

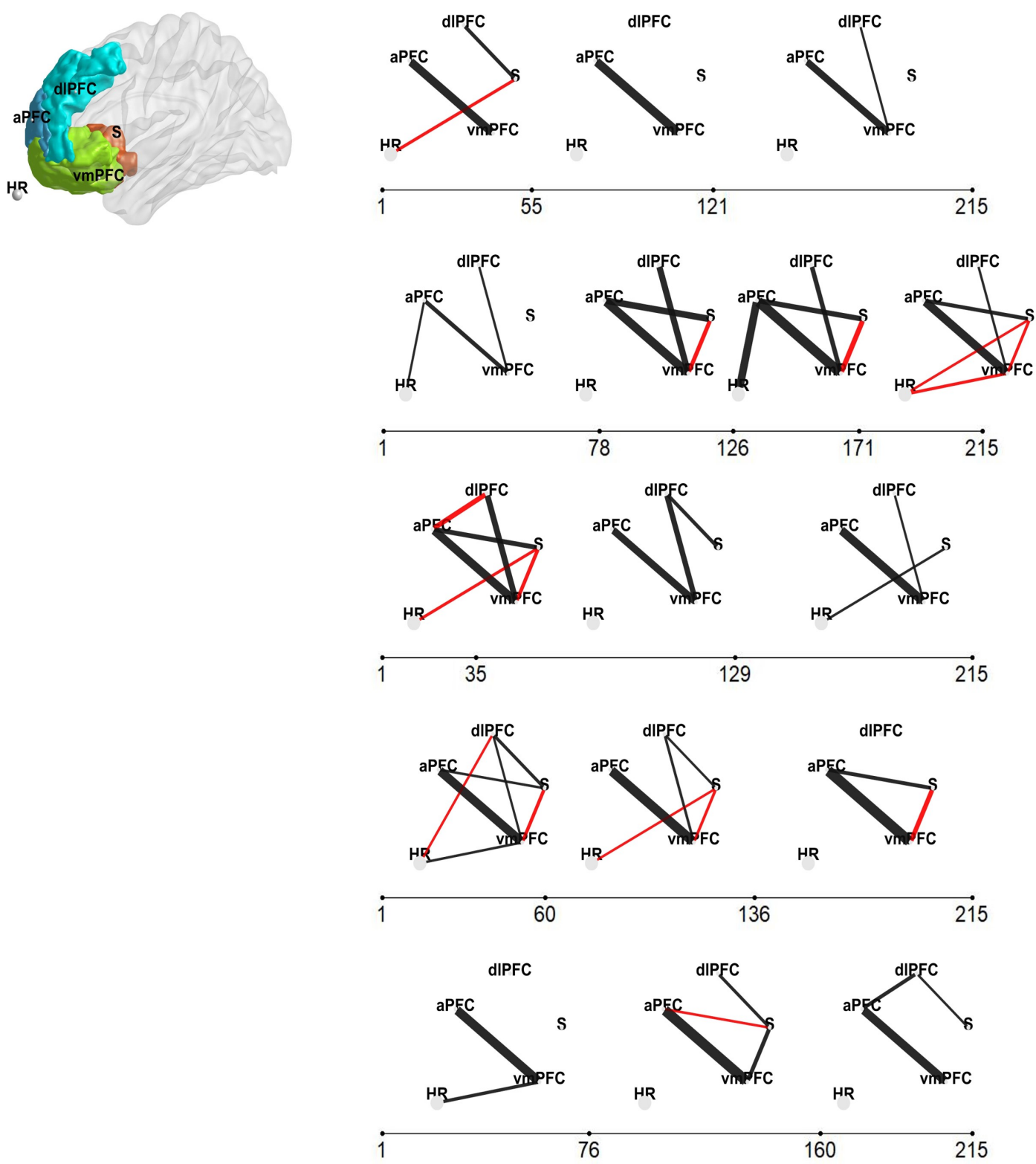

Figure 11: The four-ROI and heart rate data set (task based fMRI data set) the regions are : (1) VMPFC, (2) anterior mPFC, (3) Striatum/pallidum, and (4) DLPFC/IFJ and 5) heart rate (left). The FC undirected graphs, estimated by SCAD and BIC, for subjects 4, 6, 8, 9 and 11 (each row corresponds to a particular subject) between the detected change-points for CCID. $L_{\infty}$ (right). Black (red) edges infer positive (negative) connectivity, and the strength of connection between the regions is directly related to the thickness of the edges, that is, the thicker the edge the stronger the connection.

in states for short periods whereas others transition more quickly. Hence, CCID has a major advantage over moving window-type methods as we do not have to choose the window length, which can have significant consequences on the estimated brain networks. As we have already stated for the Anxiety fMRI data, one of 

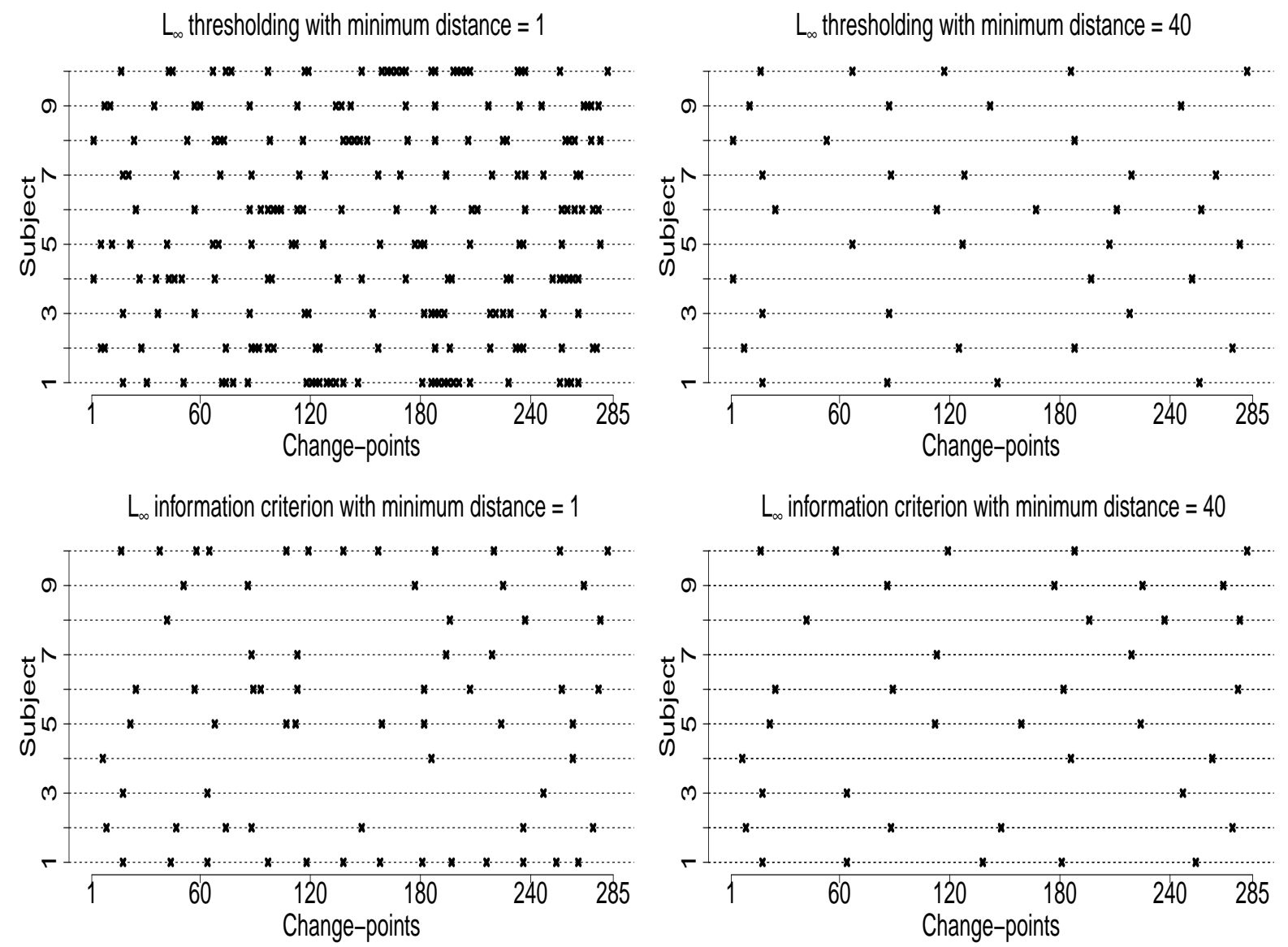

Figure 12: The detected change-points for the resting state fMRI data using CCID with threshold $L_{\infty}$ (top) and information criterion $L_{\infty} I C$ (bottom) using a minimum distance between change-points of $\delta=1$ (left) and $\delta=40$ (right). The $\mathrm{y}$-axis depicts the subject number while the $\mathrm{x}$-axis shows the change-point location times.

the main advantages of CCID over previous change-point methods is that CCID allows for detection in the presence of frequent changes of possibly small magnitudes. Hence, for resting state data, where the subjects are unconstrained, CCID is very suitable. No previous method has been able to detect as many change-points and also change-points that are very close to one another. In some studies, the number of change-points itself could be the objective of the study or an input into a model for disease classification. Hence, a small minimum distance could be utilized. However, in other cases, often researchers would like to estimate a partition specific brain network or the undirected graph between each pair of detected change-points for visualizing the FC network. Hence, Figure 12 (right) shows the results where a minimum distance of $\delta=40$ was used. In this case, every subject has up to 5 change-points with a minimum of 3 change-points.

Figure 13 shows the detected change-points for the resting state fMRI data using CCID with threshold $L_{2}$ (top) and information criterion $L_{2} I C$ (bottom) using a minimum distance between change-points of $\delta=1$ (left) and $\delta=40$ (right). All of the conclusions that we described above in Figure 12 are similar for these 

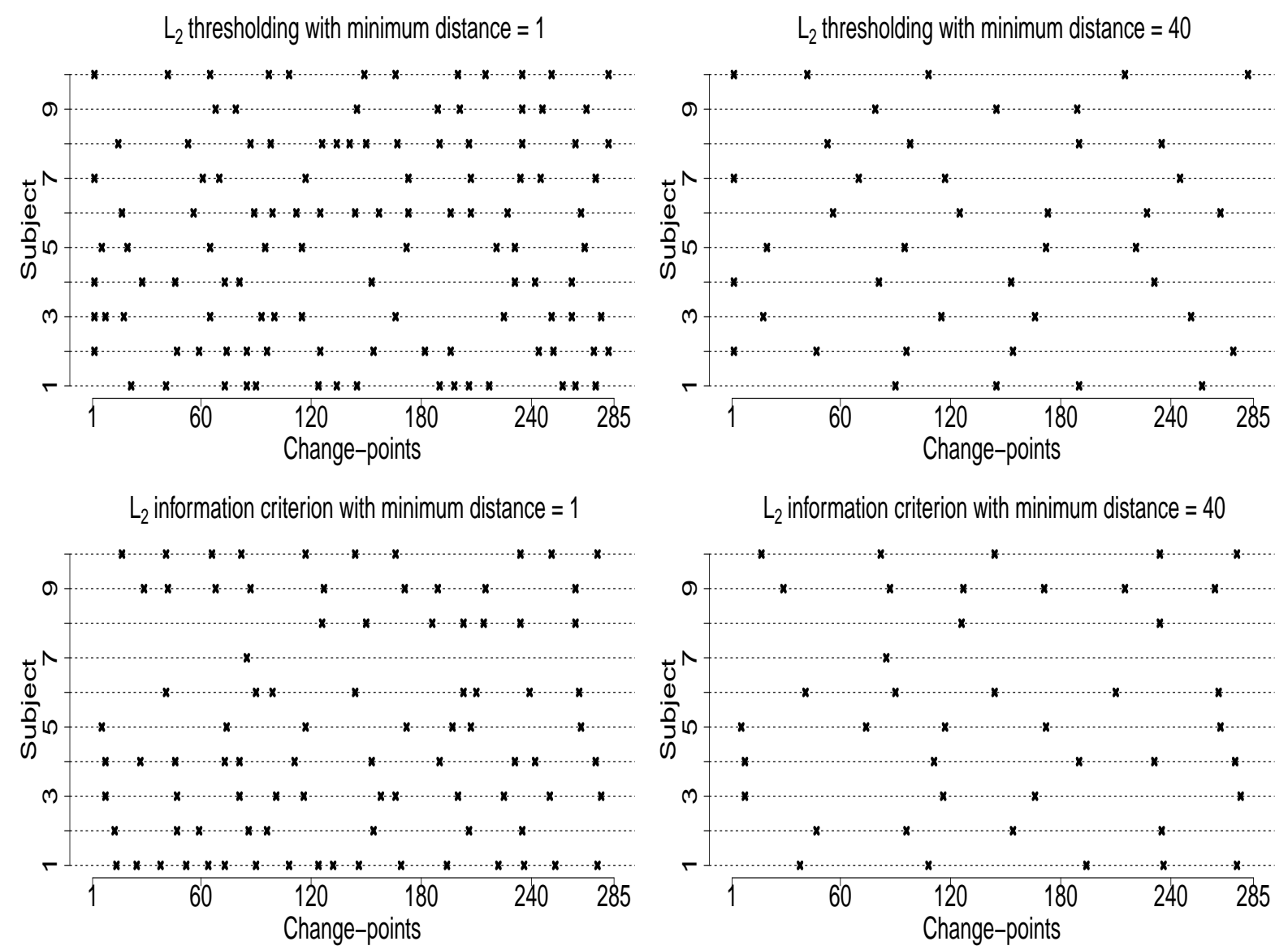

Figure 13: The detected change-points for the resting state fMRI data using CCID with threshold $L_{2}$ (top) and information criterion $L_{2} I C$ (bottom) using a minimum distance between change-points of $\delta=1$ (left) and $\delta=40$ (right). The $\mathrm{y}$-axis depicts the subject number while the $\mathrm{x}$-axis shows the change-point location times.

methods. The only difference is that CCID with threshold $L_{2}$ (top) and information criterion $L_{2} I C$ detect less change points for both $\delta=1,40$ and are more spread out for $\delta=1$.

For comparison, Figure 15 (see Appendix) shows the results for all the competing methods for the resting state fMRI data. SBS finds either one or two change-points for each subject, Factor common finds at most one change-point, while Factor idiosyncratic does not find any change-points for all subjects. The BO method finds multiple change-points for all subjects, apart from Subject 4, where this method detects one change-point. Although we are not certain of the number of change-points for resting state fMRI, as the subject is unconstrained, we expect subjects to traverse several functional states across the experiment and previous studies Ofori-Boateng et al. (2020) have located multiple change-points, something which agrees with the results of CCID and BO, but not with those of SBS and Factor. However, due to the liberal Type I errors for BO (also seen in Ofori-Boateng et al. 2020), many of BO's change-points may be due to false positive change-points. 


\section{Discussion}

\subsection{Impact of autocorrelation}

In our simulations and fMRI data analysis, we show that CCID performs well for independent data and data with a moderate amount of autocorrelation. However, in cases where the autocorrelation in the obtained periodograms is very high, we propose the following variants of our algorithm that can be employed to achieve better practical performance.

Subsampling. Suppose we have $d$ periodograms after the wavelet transformation is applied on the original $p$ time series, then we can subsample from the periodograms and apply CCID to the data sequences created. For conceptual simplicity, allow us to explain this subsampling variant through an example where the length

of each periodogram, $\left\{W_{t}^{(q)}\right\}_{t=1,2, \ldots, T}$ for $q=1,2, \ldots, d$, is $T=1000$. In this example, we subsample every $s=4$ data points; of course different values of $s$ can be taken and our specific choice is made only for the sake of explaining this subsampling variant. We note that the lower the value of $s$, the more autocorrelation in our new (subsampled) data but also the more accurate the estimated change-point locations. In our example of $T=1000$ and $s=4$, the following $s$ data sequences for each periodogram are created:

$$
\begin{aligned}
& \left\{W_{t}^{(q), 1}\right\}_{t=1,2, \ldots, 250}=\left\{W_{1}^{(q)}, W_{5}^{(q)}, \ldots, W_{997}^{(q)}\right\} \\
& \left\{W_{t}^{(q), 2}\right\}_{t=1,2, \ldots, 250}=\left\{W_{2}^{(q)}, W_{6}^{(q)}, \ldots, W_{998}^{(q)}\right\} \\
& \left\{W_{t}^{(q), 3}\right\}_{t=1,2, \ldots, 250}=\left\{W_{3}^{(q)}, W_{7}^{(q)}, \ldots, W_{999}^{(q)}\right\} \\
& \left\{W_{t}^{(q), 4}\right\}_{t=1,2, \ldots, 250}=\left\{W_{4}^{(q)}, W_{8}^{(q)}, \ldots, W_{1000}^{(q)}\right\} .
\end{aligned}
$$

After completing this step, we apply, for each $j=1,2,3,4$ separately, CCID to the multivariate time series

$$
\left\{\boldsymbol{W}_{\boldsymbol{t}}^{\boldsymbol{j}}\right\}_{t=1,2, \ldots, 250}=\left\{\begin{array}{c}
W_{t}^{(1), j} \\
W_{t}^{(2), j} \\
\vdots \\
W_{t}^{(Q), j}
\end{array}\right\}_{t=1,2, \ldots, 250}
$$

in order, for each $j=1,2, \ldots, s$, to detect the change-points for $\boldsymbol{W}_{\boldsymbol{t}}^{\boldsymbol{j}}$; this set of change-points is denoted by $H^{(j)}=\left\{\hat{r}_{1}^{(j)}, \hat{r}_{2}^{(j)}, \ldots, \hat{r}_{N_{j}}^{(j)}\right\}$. We define

$$
H=\cup_{j=1,2, \ldots, s} H^{(j)}=\left\{\hat{r}_{1}^{(1)}, \hat{r}_{2}^{(1)}, \ldots, \hat{r}_{N_{1}}^{(1)}, \hat{r}_{1}^{(2)}, \hat{r}_{2}^{(2)}, \ldots, \hat{r}_{N_{2}}^{(2)}, \ldots, \hat{r}_{1}^{(s)}, \hat{r}_{2}^{(s)}, \ldots, \hat{r}_{N_{s}}^{(s)}\right\}
$$

Some of the elements in the set $H$ can of course be identical. The next step is to apply a majority voting rule, in the sense that we only keep those values that appear at least $\eta$ times, where $\eta \in\{1,2, \ldots, s\}$. The 
value of $\eta$ can be decided upon using a priori knowledge of the particular neuroimaging data set. Once the values that appear at least $\eta$ times in $H$ are extracted, the change-points are then transformed to represent the change-point locations with respect to the original periodograms. For example if the $\hat{N}$ values $\tilde{r}_{k}, k=1,2, \ldots, \hat{N}$, appear more than $\eta$ times in $H$, then the estimated change-point locations with respect to the original data are $\hat{r}_{k}=s\left(\tilde{r}_{k}-1\right)+\left\lceil\frac{s}{2}\right\rceil$.

Pre-averaging. Pre-averaging is a strategy where data are averaged over short time periods. Here, for a given scale number $s$ and $d$ periodograms $\left\{W_{t}^{q}\right\}_{t=1,2, \ldots, T}$ and $q=1,2, \ldots, d$, we let $S^{*}=\lceil T / s\rceil$ and we define now for each $q$,

$$
\begin{aligned}
& \tilde{W}_{s^{*}}^{(q)}=\frac{1}{s} \sum_{t=\left(s^{*}-1\right) s+1}^{s^{*} s} W_{t}^{(q)}, \quad \text { for } s^{*}=1,2, \ldots, S^{*}-1 \\
& \tilde{W}_{S^{*}}^{(q)}=\left(T-\left(S^{*}-1\right) s\right)^{-1} \sum_{t=\left(S^{*}-1\right) s+1}^{T} W_{t}^{(q)}
\end{aligned}
$$

The next step is to apply CCID on

$$
\left\{\tilde{\boldsymbol{W}}_{\boldsymbol{s}^{*}}\right\}_{s^{*}=1,2, \ldots, S^{*}}=\left\{\begin{array}{c}
\tilde{W}_{s^{*}}^{(1)} \\
\tilde{W}_{s^{*}}^{(2)} \\
\vdots \\
\tilde{W}_{s^{*}}^{(Q)}
\end{array}\right\}_{s^{*}=1,2, \ldots, S^{*}}
$$

to obtain the estimated change-points, namely $\tilde{\tilde{r}}_{1}, \tilde{\tilde{r}}_{2}, \ldots, \tilde{\tilde{r}}_{\hat{N}}$, in increasing order. To estimate the original locations of the change-points we define $\hat{r}_{k}=\left(\tilde{\tilde{r}}_{k}-1\right) s+\left\lceil\frac{s}{2}\right\rceil, k=1,2, \ldots, \hat{N}$. There is a trade-off in the choice of the scaling parameter $s$. If the value of $s$ is large, then we assume that our new (pre-averaged) data are less autocorrelated but the amount of pre-processing obviously increases, which results in loss in power (due to less data) and also possibly in lack in the accuracy of the detected change-point locations.

\subsection{CCID constants and recommendations}

For CCID, we provided results for the $L_{2}$ and the $L_{\infty}$ threshold approaches and the $L_{2}$ and the $L_{\infty}$ information criteria. Our choice of constants for the approaches $\left(c_{1}\right.$ for the $L_{2}$ threshold; $c_{2}$ for the $L_{\infty}$ threshold and $\alpha$ for the information criteria) provided a balance between specificity and sensitivity in all signals examined in our large scale simulation study and others not included. Hence, all constants have been calibrated on higher dimensions. However, it is important that CCID remains robust to alternative choices to these parameters and the practitioner has the option to obtain more change-points by decreasing these threshold value.

In our numerical experience, it appears that the $L_{\infty}$ threshold performs best for low dimensional cases 
$(P<5)$ with the $L_{2}$ threshold over detecting the change points. The reason for this is that the $L_{\infty}$ threshold boils down to just one cross covariance while the $L_{2}$ threshold aggregates over all series. Hence, the $L_{2}$ threshold works better for small changes across many series.

\section{Conclusion}

In this study, we developed a new method, called Cross-covariance isolate detect (CCID), to detect multiple change-points in the second-order (cross-covariance or network) structure of multivariate (possibly) high-dimensional time series. We assumed that the number and location of the change-points are unknown a priori. CCID first converted our multivariate time series into periodograms and cross-periodograms. To detect the change-points CCID used a scaled CUSUM statistic and aggregated across the multivariate time series by adding only those that pass a certain threshold. CCID adapted the Isolate Detect principle to find the multiple change-points. ID works by first isolating each of the true change-points within subintervals and then secondly detecting them within these subintervals. We showed using an extensive simulation study that CCID generally had higher statistical power compared to existing methodologies. Our simulation study also showed that CCID performs better than existing methods in various challenging scenarios where the subject alternates between task and rest. In comparison, binary segmentation (used by all previous change-point methods in the neuroscience literature) failed to perform as well in this scenario. In addition, using empirical task based and resting-state fMRI data, CCID provided significant superior results in terms of change-point detection to the existing methods.

We showed that CCID is also more computationally efficient than the competing methods and in many settings is at least an order of magnitude faster than them. Computational costs can be challenging at higher dimensions as the first step of our method requires the transformation of the multivariate time series to periodograms and cross-periodograms. While in theory a large number of ROIs is possible, in practice we may need to make modifications to our proposed method to accommodate a larger number of ROIs (whole brain dynamics or voxel time series). We could take advantage of parallel computing in order to speed up the method.

CCID is novel as it allows for detection in the presence of frequent changes of possibly small magnitudes, can assign change-points to one or multiple brain regions, and is computationally very fast. We also proposed new information criteria for CCID to identify the change-points. While CCID was applied to task based and resting-state fMRI data in this work, it could seamlessly be applied to Electroencephalography (EEG) or Magnetoencephalography (MEG), or electrocorticography (ECoG) data. The outputs of CCID could also be used as an input into a classification model for predicting brain disorders. Furthermore, while previous studies have attempted to find differences between controls and patients with brain disorders such as Alzheimer's disease (Hart et al. 2018), understand FC changes in cerebral palsy before and after treatment Bakhtiari 
et al. 2017), and understand the role of speech production in reading (Cummine et al., 2016) using static

FC networks, it would be very interesting to see if dynamic FC estimated via CCID provides more detailed descriptions of these complicated processes. To conclude, CCID pertains to a general setting and can also be used in a variety of situations where one wishes to study the evolution of a high dimensional network over time.

\section{Software}

The authors have released an open source $\mathrm{R}$ package by the name of ccid. The package can be downloaded from CRAN.

\section{Acknowledgements}

The work of I. Cribben was supported by the Natural Sciences and Engineering Research Council (Canada) grant RGPIN-2018-06638 and the Xerox Faculty Fellowship, Alberta School of Business. 


\section{References}

Allen, E. A., Damaraju, E., Plis, S. M., Erhardt, E. B., Eichele, T., \& Calhoun, V. D. (2014). Tracking whole-brain connectivity dynamics in the resting state. Cerebral cortex, 24, 663-676.

Amat, J., Baratta, M. V., Paul, E., Bland, S. T., Watkins, L. R., \& Maier, S. F. (2005). Medial prefrontal cortex determines how stressor controllability affects behavior and dorsal raphe nucleus. Nature neuroscience, 8, 365-371.

Amat, J., Paul, E., Watkins, L., \& Maier, S. (2008). Activation of the ventral medial prefrontal cortex during an uncontrollable stressor reproduces both the immediate and long-term protective effects of behavioral control. Neuroscience, 154, 1178-1186.

Anastasiou, A., \& Fryzlewicz, P. (2021). Detecting multiple generalized change-points by isolating single ones. Metrika, (pp. 1-34).

Arbabshirani, M. R., Damaraju, E., Phlypo, R., Plis, S., Allen, E., Ma, S., Mathalon, D., Preda, A., Vaidya, J. G., Adali, T. et al. (2014). Impact of autocorrelation on functional connectivity. Neuroimage, 102, $294-308$.

Aue, A., Hörmann, S., Horváth, L., Reimherr, M. et al. (2009). Break detection in the covariance structure of multivariate time series models. The Annals of Statistics, 37, 4046-4087.

Bakhtiari, R., Cummine, J., Reed, A., Fox, C. M., Chouinard, B., Cribben, I., \& Boliek, C. A. (2017). Changes in brain activity following intensive voice treatment in children with cerebral palsy. Human Brain Mapping, 38, 4413-4429.

Barigozzi, M., Cho, H., \& Fryzlewicz, P. (2018). Simultaneous multiple change-point and factor analysis for high-dimensional time series. Journal of Econometrics, .

Barnett, I., \& Onnela, J.-P. (2016). Change point detection in correlation networks. Scientific reports, 6, 18893.

Calhoun, V. D., Adali, T., Pearlson, G. D., \& Pekar, J. J. (2001). A method for making group inferences from functional MRI data using independent component analysis. Human Brain Mapping, 14, 140-151.

Chang, C., \& Glover, G. H. (2010). Time-frequency dynamics of resting-state brain connectivity measured with fMRI. NeuroImage, 50, 81-89.

Chen, J., \& Gupta, A. K. (1997). Testing and locating variance changepoints with application to stock prices. Journal of the American Statistical association, 92, 739-747. 
Cho, H., \& Fryzlewicz, P. (2012). Multiscale and multilevel technique for consistent segmentation of nonstationary time series. Statistica Sinica, (pp. 207-229).

Cho, H., \& Fryzlewicz, P. (2015). Multiple-change-point detection for high dimensional time series via sparsified binary segmentation. Journal of the Royal Statistical Society: Series B (Statistical Methodology), 77, 475-507.

Cribben, I., \& Fiecas, M. (2016). Functional connectivity analyses for fmri data. Handbook of Neuroimaging Data Analysis, 369.

Cribben, I., Haraldsdottir, R., Atlas, L. Y., Wager, T. D., \& Lindquist, M. A. (2012). Dynamic Connectivity Regression: determining state-related changes in brain connectivity. NeuroImage, 61, 907-920.

Cribben, I., Wager, T. D., \& Lindquist, M. A. (2013). Detecting functional connectivity change points for single subject fMRI data. Frontiers in Computational Neuroscience, 7.

Cribben, I., \& Yu, Y. (2017). Estimating whole-brain dynamics by using spectral clustering. Journal of the Royal Statistical Society: Series C (Applied Statistics), 66, 607-627.

Critchley, H. D., Corfield, D., Chandler, M., Mathias, C., \& Dolan, R. J. (2000). Cerebral correlates of autonomic cardiovascular arousal: a functional neuroimaging investigation in humans. The Journal of physiology, 523, 259-270.

Cummine, J., Cribben, I., Luu, C., Kim, E., Bakhtiari, R., Georgiou, G., \& Boliek, C. A. (2016). Understanding the role of speech production in reading: Evidence for a print-to-speech neural network using graphical analysis. Neuropsychology, 30, 385.

Dai, M., Zhang, Z., \& Srivastava, A. (2019). Discovering common change-point patterns in functional connectivity across subjects. Medical image analysis, 58, 101532.

Daubechies, I. (1992). Ten lectures on wavelets volume 61. Siam.

Debener, S., Ullsperger, M., Siegel, M., \& Engel, A. K. (2006). Single-trial EEG-fMRI reveals the dynamics of cognitive function. Trends in cognitive sciences, 10, 558-563.

Delamillieure, P., Doucet, G., Mazoyer, B., Turbelin, M.-R., Delcroix, N., Mellet, E., Zago, L., Crivello, F., Petit, L., Tzourio-Mazoyer, N. et al. (2010). The resting state questionnaire: an introspective questionnaire for evaluation of inner experience during the conscious resting state. Brain Research Bulletin, 81, 565-573.

Dette, H., \& Wied, D. (2016). Detecting relevant changes in time series models. Journal of the Royal Statistical Society: Series B (Statistical Methodology), 78, 371-394. 
Doucet, G., Naveau, M., Petit, L., Zago, L., Crivello, F., Jobard, G., Delcroix, N., Mellet, E., TzourioMazoyer, N., Mazoyer, B. et al. (2012). Patterns of hemodynamic low-frequency oscillations in the brain are modulated by the nature of free thought during rest. NeuroImage, 59, 3194-3200.

Fan, J., \& Li, R. (2001). Variable selection via nonconcave penalized likelihood and its oracle properties. Journal of the American statistical Association, 96, 1348-1360.

Fan, J., Lv, J., \& Qi, L. (2011). Sparse high-dimensional models in economics. Annual Review of Economics, 3, 291-317.

Fang, X., Li, J., \& Siegmund, D. (2020). Segmentation and estimation of change-point models: False positive control and confidence regions. The Annals of Statistics, 48, 1615-1647.

Fiecas, M., Cribben, I., Bahktiari, R., \& Cummine, J. (2017). A variance components model for statistical inference on functional connectivity networks. NeuroImage, 149, 256-266.

Fox, M. D., Snyder, A. Z., Vincent, J. L., Corbetta, M., Van Essen, D. C., \& Raichle, M. E. (2005). The human brain is intrinsically organized into dynamic, anticorrelated functional networks. Proceedings of the National Academy of Sciences of the United States of America, 102, 9673-9678.

Fryzlewicz, P. (2014). Wild binary segmentation for multiple change-point detection. The Annals of Statistics, 42, 2243-2281.

Gianaros, P. J., Van der Veen, F. M., \& Jennings, J. R. (2004). Regional cerebral blood flow correlates with heart period and high-frequency heart period variability during working-memory tasks: Implications for the cortical and subcortical regulation of cardiac autonomic activity. Psychophysiology, 41, 521-530.

Gibberd, A. J., \& Nelson, J. D. (2014). High dimensional changepoint detection with a dynamic graphical lasso. In ICASSP (pp. 2684-2688).

Habeck, C., Steffener, J., Rakitin, B., \& Stern, Y. (2012). Can the default-mode network be described with one spatial-covariance network? Brain research, 1468, 38-51.

Handwerker, D. A., Roopchansingh, V., Gonzalez-Castillo, J., \& Bandettini, P. A. (2012). Periodic changes in fMRI connectivity. NeuroImage, 63, 1712-1719.

Hart, B., Cribben, I., Fiecas, M., Initiative, A. D. N. et al. (2018). A longitudinal model for functional connectivity networks using resting-state fmri. NeuroImage, 178, 687-701.

Hutchison, R. M., Womelsdorf, T., Allen, E. A., Bandettini, P. A., Calhoun, V. D., Corbetta, M., Penna, S. D., Duyn, J., Glover, G., Gonzalez-Castillo, J. et al. (2013a). Dynamic functional connectivity: promises, issues, and interpretations. NeuroImage, 80, 360-378. 
Hutchison, R. M., Womelsdorf, T., Gati, J. S., Everling, S., \& Menon, R. S. (2013b). Resting-state networks show dynamic functional connectivity in awake humans and anesthetized macaques. Human Brain Mapping, 34, 2154-2177.

Inclan, C., \& Tiao, G. C. (1994). Use of cumulative sums of squares for retrospective detection of changes of variance. Journal of the American Statistical Association, 89, 913-923.

Jones, D. T., Vemuri, P., Murphy, M. C., Gunter, J. L., Senjem, M. L., Machulda, M. M., Przybelski, S. A., Gregg, B. E., Kantarci, K., \& Knopman, D. S. (2012). Non-stationarity in the 'resting brain's' modular architecture. Alzheimer's and Dementia, 8, 689.

Kao, C., Trapani, L., Urga, G. et al. (2018). Testing for instability in covariance structures. Bernoulli, 24, $740-771$.

Kirch, C., Muhsal, B., \& Ombao, H. (2015). Detection of changes in multivariate time series with application to EEG data. Journal of the American Statistical Association, 110, 1197-1216.

Kiviniemi, V., Vire, T., Remes, J., Elseoud, A. A., Starck, T., Tervonen, O., \& Nikkinen, J. (2011). A sliding time-window ICA reveals spatial variability of the default mode network in time. Brain Connectivity, 1, 339-347.

Kundu, S., Ming, J., Pierce, J., McDowell, J., \& Guo, Y. (2018). Estimating dynamic brain functional networks using multi-subject fmri data. NeuroImage, 183, 635-649.

Leonardi, N., Richiardi, J., Gschwind, M., Simioni, S., Annoni, J.-M., Schluep, M., Vuilleumier, P., \& Van De Ville, D. (2013). Principal components of functional connectivity: A new approach to study dynamic brain connectivity during rest. NeuroImage, 83, 937-950.

Li, Y.-N., Li, D., \& Fryzlewicz, P. (2019). Detection of multiple structural breaks in large covariance matrices. Preprint on webpage at stats.lse.ac.uk/fryzlewicz/wbscov/wbscov.pdf

Lindquist, M. A., Yuting, X., Nebel, M. B., \& Caffo, B. S. (2014). Evaluating dynamic bivariate correlations in resting-state fMRI: A comparison study and a new approach. NeuroImage, 101, 531-546.

Ofori-Boateng, D., Gel, Y. R., \& Cribben, I. (2020). Nonparametric anomaly detection on time series of graphs. Journal of Computational and Graphical Statistics, 0, 1-26.

Ondrus, M., Olds, E., \& Cribben, I. (2021). Factorized binary search: change point detection in the network structure of multivariate high-dimensional time series. 
Rozanski, A., Bairey, C. N., Krantz, D. S., Friedman, J., Resser, K. J., Morell, M., Hilton-Chalfen, S., Hestrin, L., Bietendorf, J., \& Berman, D. S. (1988). Mental stress and the induction of silent myocardial ischemia in patients with coronary artery disease. New England Journal of Medicine, 318, 1005-1012.

Sakoğlu, U., Pearlson, G. D., Kiehl, K. A., Wang, Y. M., Michael, A. M., \& Calhoun, V. D. (2010). A method for evaluating dynamic functional network connectivity and task-modulation: application to schizophrenia. Magnetic Resonance Materials in Physics, Biology and Medicine, 23, 351-366.

Schröder, A. L., \& Ombao, H. (2019). Fresped: Frequency-specific change-point detection in epileptic seizure multi-channel eeg data. Journal of the American Statistical Association, 114, 115-128.

Smith, S. M., Miller, K. L., Salimi-Khorshidi, G., Webster, M., Beckmann, C. F., Nichols, T. E., Ramsey, J. D., \& Woolrich, M. W. (2011). Network modelling methods for fmri. Neuroimage, 54, 875-891.

Sundararajan, R. R., \& Pourahmadi, M. (2018). Nonparametric change point detection in multivariate piecewise stationary time series. Journal of Nonparametric Statistics, (pp. 1-31).

Tzourio-Mazoyer, N., Landeau, B., Papathanassiou, D., Crivello, F., Etard, O., Delcroix, N., Mazoyer, B., \& Joliot, M. (2002). Automated anatomical labeling of activations in spm using a macroscopic anatomical parcellation of the mni mri single-subject brain. NeuroImage, 15, 273-289.

Vidakovic, B. (2009). Statistical modeling by wavelets volume 503. John Wiley \& Sons.

Wager, T. D., Waugh, C. E., Lindquist, M., Noll, D. C., Fredrickson, B. L., \& Taylor, S. F. (2009). Brain mediators of cardiovascular responses to social threat: part i: reciprocal dorsal and ventral sub-regions of the medial prefrontal cortex and heart-rate reactivity. Neuroimage, 47, 821-835.

Woods, R. P., Grafton, S. T., Holmes, C. J., Cherry, S. R., \& Mazziotta, J. C. (1998). Automated image registration: I. general methods and intrasubject, intramodality validation. Journal of computer assisted tomography, 22, 139-152.

Xiong, X., \& Cribben, I. (2021). Beyond linear dynamic functional connectivity: a vine copula change point model. bioRxiv, .

Xu, M., Reiss, P. T., \& Cribben, I. (2020). Generalized reliability based on distances. Biometrics, .

Zhang, J., Li, X., Li, C., Lian, Z., Huang, X., Zhong, G., Zhu, D., Li, K., Jin, C., Hu, X., Han, J., Guo, L., Hu, X., Li, L., \& Liu, T. (2014). Inferring functional interaction and transition patterns via dynamic bayesian variable partition models. Human Brain Mapping, 35, 3314-3331.

Zhu, Y., \& Cribben, I. (2018). Sparse graphical models for functional connectivity networks: best methods and the autocorrelation issue. Brain connectivity, 8, 139-165. 
bioRxiv preprint doi: https://doi.org/10.1101/2020.12.20.423696; this version posted June 8, 2021. The copyright holder for this preprint (which was not certified by peer review) is the author/funder, who has granted bioRxiv a license to display the preprint in perpetuity. It is made available under aCC-BY-NC-ND 4.0 International license.

Zou, C., Yin, G., Feng, L., Wang, Z. et al. (2014). Nonparametric maximum likelihood approach to multiple change-point problems. The Annals of Statistics, 42, 970-1002. 


\section{Appendix}

\section{A The results for the real data presented in tables}

Table 11: The estimated number and location of the change-points for the 4 ROIs and heart rate task based fMRI data set for Sparsified Binary Segmentation (SBS), Factor with common components (Factor com), Factor with idiosyncratic components (Factor id), Barnett \& Onnela (2016) (BO), and Cross-covariance isolate detect with the $L_{2}$ threshold (CCID. $\left.L_{2}\right)$, the $L_{\infty}$ threshold (CCID. $L_{\infty}$ ), the $L_{2}$ information criterion (CCID. $L_{2} I C$ ) and the $L_{\infty}$ information criterion (CCID. $L_{\infty} I C$ ). A $\delta$ in the name of a method indicates a minimum distance between change-points of 40 time points was enforced. The computational times for each method are also provided.

\begin{tabular}{|c|c|c|c|c|}
\hline ID & Method & Number & $\begin{array}{l}\text { Estimated } \\
\text { Locations }\end{array}$ & Time $(\mathrm{s})$ \\
\hline \multirow{12}{*}{1} & SBS & 0 & - & 1.51 \\
\hline & Factor com & 0 & - & 2.19 \\
\hline & Factor id & 1 & 43 & 2.19 \\
\hline & $\mathrm{BO}$ & 1 & 182 & 10.19 \\
\hline & CCID. $L_{2}$ & 8 & $(34,70,78,115,116,137,181,185)$ & 0.02 \\
\hline & CCID. $L_{\infty}$ & 3 & $(70,80,138)$ & 0.01 \\
\hline & CCID. $L_{2} I C$ & 12 & $(36,61,78,86,113,117,128,138,173,175,185,209)$ & 0.05 \\
\hline & CCID. $L_{\infty} I C$ & 2 & $(138,180)$ & 0.02 \\
\hline & $\delta$ CCID. $L_{2}$ & 3 & $(70,137,185)$ & 0.02 \\
\hline & $\delta$ CCID. $L_{\infty}$ & 2 & $(70,138)$ & 0.01 \\
\hline & $\delta$ CCID. $L_{2} I C$ & 3 & $(61,138,185)$ & 0.05 \\
\hline & $\delta$ CCID. $L_{\infty} I C$ & 2 & $(138,180)$ & 0.02 \\
\hline \multirow{12}{*}{2} & SBS & 0 & - & 1.37 \\
\hline & Factor com & 0 & - & 2.33 \\
\hline & Factor id & 1 & 161 & 2.33 \\
\hline & $\mathrm{BO}$ & 0 & - & 5.55 \\
\hline & CCID. $L_{2}$ & 7 & $(28,60,65,126,133,170,175)$ & 0.05 \\
\hline & CCID. $L_{\infty}$ & 2 & $(130,133)$ & 0.01 \\
\hline & CCID. $L_{2} I C$ & 2 & $(170,175)$ & 0.01 \\
\hline & CCID. $L_{\infty} I C$ & 0 & - & 0.02 \\
\hline & $\delta$ CCID.$L_{2}$ & 2 & $(65,133)$ & 0.02 \\
\hline & $\delta$ CCID. $L_{\infty}$ & 1 & 133 & 0.01 \\
\hline & $\delta$ CCID.$L_{2} I C$ & 1 & 170 & 0.05 \\
\hline & $\delta$ CCID. $L_{\infty} I C$ & 0 & - & 0.02 \\
\hline \multirow{12}{*}{3} & SBS & 0 & - & 1.47 \\
\hline & Factor com & 0 & - & 3.47 \\
\hline & Factor id & 0 & - & 3.47 \\
\hline & $\mathrm{BO}$ & 0 & - & 5.67 \\
\hline & CCID. $L_{2}$ & 5 & $(19,20,73,112,134)$ & 0.02 \\
\hline & CCID. $L_{\infty}$ & 1 & 133 & 0.02 \\
\hline & CCID. $L_{2} \mathrm{IC}$ & 2 & $(73,209)$ & 0.04 \\
\hline & CCID. $L_{\infty} \mathrm{IC}$ & 0 & - & 0.09 \\
\hline & $\delta$ CCID. $L_{2}$ & 3 & $(19,73,134)$ & 0.02 \\
\hline & $\delta$ CCID.$L_{\infty}$ & 1 & 133 & 0.01 \\
\hline & $\delta$ CCID.$L_{2} I C$ & 2 & $(73,209)$ & 0.05 \\
\hline & $\delta$ CCID $L_{\infty} I C$ & 0 & - & 0.02 \\
\hline
\end{tabular}


bioRxiv preprint doi: https://doi.org/10.1101/2020.12.20.423696; this version posted June 8, 2021. The copyright holder for this preprint (which was not certified by peer review) is the author/funder, who has granted bioRxiv a license to display the preprint in perpetuity. It is made available under aCC-BY-NC-ND 4.0 International license.

\begin{tabular}{|c|c|c|c|c|}
\hline ID & Method & Number & $\begin{array}{l}\text { Estimated } \\
\text { Locations }\end{array}$ & Time $(\mathrm{s})$ \\
\hline \multirow{12}{*}{4} & SBS & 1 & 124 & 1.36 \\
\hline & Factor com & 1 & 125 & 3.30 \\
\hline & Factor id & 1 & 35 & 3.30 \\
\hline & $\mathrm{BO}$ & 0 & - & 5.16 \\
\hline & CCID. $L_{2}$ & 7 & $(20,27,75,76,102,121,149)$ & 0.01 \\
\hline & CCID. $L_{\infty}$ & 3 & $(55,101,121)$ & 0.02 \\
\hline & CCID. $L_{2} \mathrm{IC}$ & 11 & $(20,27,28,55,75,76,102,105,121,151,209)$ & 0.05 \\
\hline & CCID. $L_{\infty} \mathrm{IC}$ & 3 & $(55,101,121)$ & 0.02 \\
\hline & $\delta$ CCID. $L_{2}$ & 2 & $(20,121)$ & 0.02 \\
\hline & $\delta$ CCID.$L_{\infty}$ & 2 & $(55,121)$ & 0.01 \\
\hline & $\delta$ CCID. $L_{2} I C$ & 2 & $(121,209)$ & 0.05 \\
\hline & $\delta$ CCID. $L_{\infty} I C$ & 2 & $(55,121)$ & 0.02 \\
\hline \multirow{12}{*}{5} & SBS & 0 & - & 1.47 \\
\hline & Factor com & 0 & - & 3.52 \\
\hline & Factor id & 0 & - & 3.52 \\
\hline & $\mathrm{BO}$ & 0 & - & 5.04 \\
\hline & CCID. $L_{2}$ & 6 & $(13,60,62,68,70,208)$ & 0.03 \\
\hline & CCID. $L_{\infty}$ & 3 & $(60,63,68)$ & 0.02 \\
\hline & CCID. $L_{2} \mathrm{IC}$ & 2 & $(57,70)$ & 0.03 \\
\hline & CCID. $L_{\infty} \mathrm{IC}$ & 2 & $(60,68)$ & 0.03 \\
\hline & $\delta$ CCID. $L_{2}$ & 3 & $(13,70,208)$ & 0.02 \\
\hline & $\delta$ CCID.$L_{\infty}$ & 1 & 68 & 0.01 \\
\hline & $\delta$ CCID. $L_{2} I C$ & 1 & 70 & 0.05 \\
\hline & $\delta$ CCID. $L_{\infty} I C$ & 1 & 68 & 0.02 \\
\hline \multirow{12}{*}{6} & SBS & $\overline{1} 1$ & 73 & 1.41 \\
\hline & Factor com & 0 & - & 3.69 \\
\hline & Factor id & 1 & 84 & 3.69 \\
\hline & $\mathrm{BO}$ & 1 & 170 & 10.05 \\
\hline & CCID. $L_{2}$ & 12 & $(44,46,78,80,112,118,122,131,157,167,171,193)$ & 0.02 \\
\hline & CCID. $L_{\infty}$ & 6 & $(78,118,124,126,167,171)$ & 0.01 \\
\hline & CCID. $L_{2} \mathrm{IC}$ & 5 & $(78,118,126,167,171)$ & 0.06 \\
\hline & CCID. $L_{\infty} \mathrm{IC}$ & 10 & $(78,80,96,118,122,131,157,167,171,208)$ & 0.03 \\
\hline & $\delta$ CCID. $L_{2}$ & 2 & $(78,171)$ & 0.02 \\
\hline & $\delta$ CCID. $L_{\infty}$ & 3 & $(78,126,171)$ & 0.01 \\
\hline & $\delta$ CCID. $L_{2} I C$ & 2 & $(78,171)$ & 0.05 \\
\hline & $\delta$ CCID. $L_{\infty} I C$ & 3 & $(78,126,171)$ & 0.02 \\
\hline \multirow{12}{*}{7} & SBS & 0 & - & 1.42 \\
\hline & Factor com & 1 & 78 & 3.23 \\
\hline & Factor id & 1 & 78 & 3.23 \\
\hline & $\mathrm{BO}$ & 0 & - & 13.56 \\
\hline & CCID. $L_{2}$ & 7 & $(60,63,76,92,150,164,165)$ & 0.01 \\
\hline & CCID. $L_{\infty}$ & 1 & 76 & 0.03 \\
\hline & CCID. $L_{2} \mathrm{IC}$ & 2 & $(60,76)$ & 0.03 \\
\hline & CCID. $L_{\infty} \mathrm{IC}$ & 2 & $(64,76)$ & 0.01 \\
\hline & $\delta$ CCID. $L_{2}$ & 2 & $(76,150)$ & 0.02 \\
\hline & $\delta$ CCID.$L_{\infty}$ & 1 & 76 & 0.01 \\
\hline & $\delta$ CCID. $L_{2} I C$ & 1 & 76 & 0.05 \\
\hline & $\delta$ CCID. $L_{\infty} I C$ & 1 & 76 & 0.02 \\
\hline
\end{tabular}


bioRxiv preprint doi: https://doi.org/10.1101/2020.12.20.423696; this version posted June 8, 2021. The copyright holder for this preprint (which was not certified by peer review) is the author/funder, who has granted bioRxiv a license to display the preprint in perpetuity. It is made available under aCC-BY-NC-ND 4.0 International license.

\begin{tabular}{|c|c|c|c|c|}
\hline ID & Method & Number & $\begin{array}{l}\text { Estimated } \\
\text { Locations }\end{array}$ & Time $(\mathrm{s})$ \\
\hline \multirow{12}{*}{8} & SBS & 0 & - & 1.40 \\
\hline & Factor com & 0 & - & 3.38 \\
\hline & Factor id & 0 & - & 3.38 \\
\hline & $\mathrm{BO}$ & 2 & $(102,145)$ & 5.37 \\
\hline & CCID. $L_{2}$ & 9 & $(35,38,63,70,98,112,130,185,195)$ & 0.02 \\
\hline & CCID. $L_{\infty}$ & 4 & $(35,38,65,129)$ & 0.02 \\
\hline & CCID. $L_{2} \mathrm{IC}$ & 12 & $(35,38,63,70,98,112,130,145,169,185,196,209)$ & 0.04 \\
\hline & CCID. $L_{\infty} \mathrm{IC}$ & 4 & $(35,38,63,70)$ & 0.02 \\
\hline & $\delta$ CCID. $L_{2}$ & 3 & $(35,130,195)$ & 0.02 \\
\hline & $\delta$ CCID. $L_{\infty}$ & 2 & $(35,129)$ & 0.01 \\
\hline & $\delta$ CCID. $L_{2} I C$ & 3 & $(35,145,196)$ & 0.05 \\
\hline & $\delta$ CCID. $L_{\infty} I C$ & 2 & $(35,129)$ & 0.02 \\
\hline \multirow{12}{*}{9} & SBS & 0 & - & 1.45 \\
\hline & Factor com & 2 & $(59,141)$ & 3.72 \\
\hline & Factor id & 0 & - & 3.72 \\
\hline & $\mathrm{BO}$ & 1 & 125 & 5.37 \\
\hline & CCID. $L_{2}$ & 12 & $(30,33,59,75,105,108,123,136,164,165,204,207)$ & 0.01 \\
\hline & CCID. $L_{\infty}$ & 4 & $(60,74,125,136)$ & 0.01 \\
\hline & CCID. $L_{2} \mathrm{IC}$ & 4 & $(59,75,123,136)$ & 0.04 \\
\hline & CCID. $L_{\infty} \mathrm{IC}$ & 4 & $(60,74,125,136)$ & 0.01 \\
\hline & $\delta$ CCID. $L_{2}$ & 3 & $(59,136,204)$ & 0.02 \\
\hline & $\delta$ CCID.$L_{\infty}$ & 2 & $(60,136)$ & 0.01 \\
\hline & $\delta$ CCID. $L_{2} I C$ & 2 & $(59,136)$ & 0.05 \\
\hline & $\delta$ CCID. $L_{\infty} I C$ & 2 & $(60,136)$ & 0.02 \\
\hline \multirow{12}{*}{10} & SBS & 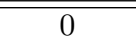 & - & 1.58 \\
\hline & Factor com & 0 & - & 3.34 \\
\hline & Factor id & 0 & - & 3.34 \\
\hline & $\mathrm{BO}$ & 2 & $(61,67)$ & 12.51 \\
\hline & CCID. $L_{2}$ & 5 & $(9,60,63,71,139)$ & 0.01 \\
\hline & CCID. $L_{\infty}$ & 1 & 71 & 0.02 \\
\hline & CCID. $L_{2} \mathrm{IC}$ & 3 & $(60,63,71)$ & 0.03 \\
\hline & CCID. $L_{\infty} \mathrm{IC}$ & 0 & - & 0.03 \\
\hline & $\delta$ CCID. $L_{2}$ & 3 & $(9,71,139)$ & 0.02 \\
\hline & $\delta$ CCID. $L_{\infty}$ & 1 & 71 & 0.01 \\
\hline & $\delta$ CCID. $L_{2} I C$ & 1 & 71 & 0.05 \\
\hline & $\delta$ CCID. $L_{\infty} I C$ & 0 & - & 0.02 \\
\hline \multirow{12}{*}{11} & SBS & 0 & - & 1.52 \\
\hline & Factor com & 0 & - & 4.05 \\
\hline & Factor id & 0 & - & 4.05 \\
\hline & $\mathrm{BO}$ & 0 & - & 5.12 \\
\hline & CCID. $L_{2}$ & 7 & $(31,59,88,127,146,164,210)$ & 0.01 \\
\hline & CCID. $L_{\infty}$ & 2 & $(76,160)$ & 0.02 \\
\hline & CCID. $L_{2} \mathrm{IC}$ & 3 & $(59,146,169)$ & 0.03 \\
\hline & CCID. $L_{\infty} \mathrm{IC}$ & 1 & 160 & 0.02 \\
\hline & $\delta$ CCID. $L_{2}$ & 3 & $(31,164,210)$ & 0.02 \\
\hline & $\delta$ CCID. $L_{\infty}$ & 2 & 76,160 & 0.01 \\
\hline & $\delta$ CCID. $L_{2} I C$ & 2 & 59,169 & 0.05 \\
\hline & $\delta$ CCID. $L_{\infty} I C$ & 1 & 160 & 0.02 \\
\hline
\end{tabular}


bioRxiv preprint doi: https://doi.org/10.1101/2020.12.20.423696; this version posted June 8, 2021. The copyright holder for this preprint (which was not certified by peer review) is the author/funder, who has granted bioRxiv a license to display the preprint in perpetuity. It is made available under aCC-BY-NC-ND 4.0 International license.

\begin{tabular}{|c|c|c|c|c|}
\hline ID & Method & Number & $\begin{array}{l}\text { Estimated } \\
\text { Locations }\end{array}$ & Time $(\mathrm{s})$ \\
\hline \multirow{12}{*}{12} & SBS & 1 & 109 & 1.49 \\
\hline & Factor com & 1 & 96 & 2.80 \\
\hline & Factor id & 0 & - & 2.80 \\
\hline & $\mathrm{BO}$ & 0 & - & 10.26 \\
\hline & CCID. $L_{2}$ & 11 & $(2,49,69,70,94,114,128,149,185,195,201)$ & 0.02 \\
\hline & CCID. $L_{\infty}$ & 5 & $(2,59,66,97,195)$ & 0.02 \\
\hline & CCID. $L_{2} \mathrm{IC}$ & 5 & $(2,49,114,167,193)$ & 0.05 \\
\hline & CCID. $L_{\infty} \mathrm{IC}$ & 3 & $(2,59,114)$ & 0.03 \\
\hline & $\delta$ CCID. $L_{2}$ & 4 & $(2,49,114,195)$ & 0.02 \\
\hline & $\delta$ CCID.$L_{\infty}$ & 3 & $(2,66,195)$ & 0.01 \\
\hline & $\delta$ CCID. $L_{2} I C$ & 4 & $(2,49,114,193)$ & 0.05 \\
\hline & $\delta$ CCID. $L_{\infty} I C$ & 3 & $(2,59,114)$ & 0.02 \\
\hline \multirow{12}{*}{13} & SBS & 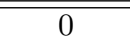 & - & 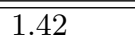 \\
\hline & Factor com & 0 & - & 2.83 \\
\hline & Factor id & 0 & - & 2.83 \\
\hline & $\mathrm{BO}$ & 0 & - & 5.17 \\
\hline & CCID. $L_{2}$ & 4 & $(7,112,114,195)$ & 0.02 \\
\hline & CCID. $L_{\infty}$ & 0 & - & 0.02 \\
\hline & CCID. $L_{2} \mathrm{IC}$ & 0 & - & 0.02 \\
\hline & CCID. $L_{\infty} \mathrm{IC}$ & 0 & - & 0.02 \\
\hline & $\delta$ CCID. $L_{2}$ & 3 & $(7,112,195)$ & 0.02 \\
\hline & $\delta$ CCID. $L_{\infty}$ & 0 & - & 0.01 \\
\hline & $\delta$ CCID. $L_{2} I C$ & 0 & - & 0.05 \\
\hline & $\delta$ CCID.$L_{\infty} I C$ & 0 & - & 0.02 \\
\hline \multirow{12}{*}{14} & SBS & 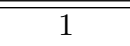 & 155 & 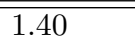 \\
\hline & Factor com & 1 & 151 & 3.20 \\
\hline & Factor id & 1 & 168 & 3.20 \\
\hline & $\mathrm{BO}$ & 1 & 153 & 10.29 \\
\hline & CCID. $L_{2}$ & 8 & $(12,16,68,70,74,125,198,202)$ & 0.02 \\
\hline & CCID. $L_{\infty}$ & 2 & $(150,192)$ & 0.04 \\
\hline & CCID. $L_{2} \mathrm{IC}$ & 4 & $(92,125,202,207)$ & 0.03 \\
\hline & CCID. $L_{\infty} \mathrm{IC}$ & 2 & $(134,192)$ & 0.03 \\
\hline & $\delta$ CCID. $L_{2}$ & 3 & $(12,74,125,198)$ & 0.02 \\
\hline & $\delta$ CCID. $L_{\infty}$ & 2 & $(150,192)$ & 0.01 \\
\hline & $\delta$ CCID. $L_{2} I C$ & 2 & $(125,202)$ & 0.05 \\
\hline & $\delta$ CCID. $L_{\infty} I C$ & 2 & $(134,192)$ & 0.02 \\
\hline \multirow{12}{*}{15} & SBS & 1 & 138 & 1.61 \\
\hline & Factor com & 3 & $(31,111,138)$ & 3.10 \\
\hline & Factor id & 0 & - & 3.10 \\
\hline & $\mathrm{BO}$ & 1 & 134 & 10.03 \\
\hline & CCID. $L_{2}$ & 6 & $(60,63,75,110,131,138)$ & 0.02 \\
\hline & CCID. $L_{\infty}$ & 1 & 137 & 0.01 \\
\hline & CCID. $L_{2} \mathrm{IC}$ & 3 & $(75,110,155)$ & 0.03 \\
\hline & CCID. $L_{\infty} \mathrm{IC}$ & 1 & 137 & 0.01 \\
\hline & $\delta \mathrm{CCID} L_{2}$ & 1 & 138 & 0.02 \\
\hline & $\delta$ CCID. $L_{\infty}$ & 1 & 137 & 0.01 \\
\hline & $\delta$ CCID. $L_{2} I C$ & 2 & $(110,155)$ & 0.05 \\
\hline & $\delta$ CCID.$L_{\infty} I C$ & 1 & 137 & 0.02 \\
\hline
\end{tabular}


bioRxiv preprint doi: https://doi.org/10.1101/2020.12.20.423696; this version posted June 8, 2021. The copyright holder for this preprint (which was not certified by peer review) is the author/funder, who has granted bioRxiv a license to display the preprint in perpetuity. It is made available under aCC-BY-NC-ND 4.0 International license.

\begin{tabular}{|c|c|c|c|c|}
\hline ID & Method & Number & $\begin{array}{l}\text { Estimated } \\
\text { Locations }\end{array}$ & Time (s) \\
\hline \multirow{12}{*}{16} & SBS & 0 & - & 1.47 \\
\hline & Factor com & 0 & - & 2.98 \\
\hline & Factor id & 0 & - & 2.98 \\
\hline & $\mathrm{BO}$ & 0 & - & 10.12 \\
\hline & CCID. $L_{2}$ & 16 & $(14,25,59,79,105,108,117,130,132,138,184,185,195,196,198,205)$ & 0.01 \\
\hline & CCID. $L_{\infty}$ & 5 & $(65,68,108,196,205)$ & 0.01 \\
\hline & CCID. $L_{2} \mathrm{IC}$ & 10 & $(25,59,90,93,105,108,138,195,198,205)$ & 0.07 \\
\hline & CCID. $L_{\infty} \mathrm{IC}$ & 2 & $(65,108)$ & 0.02 \\
\hline & $\delta$ CCID. $L_{2}$ & 3 & $(59,138,205)$ & 0.02 \\
\hline & $\delta$ CCID. $L_{\infty}$ & 3 & $(65,108,205)$ & 0.01 \\
\hline & $\delta \mathrm{CCID} . L_{2} I C$ & 3 & $(59,108,195)$ & 0.05 \\
\hline & $\delta$ CCID. $L_{\infty} I C$ & 2 & $(65,108)$ & 0.02 \\
\hline \multirow{12}{*}{17} & SBS & 0 & - & 1.45 \\
\hline & Factor com & 1 & 188 & 3.09 \\
\hline & Factor id & 1 & 187 & 3.09 \\
\hline & $\mathrm{BO}$ & 2 & $(111,190)$ & 14.48 \\
\hline & CCID. $L_{2}$ & 10 & $(9,10,63,74,108,109,156,187,189,195)$ & 0.02 \\
\hline & CCID. $L_{\infty}$ & 6 & $(62,70,125,154,187,189)$ & 0.01 \\
\hline & CCID. $L_{2} \mathrm{IC}$ & 12 & $(9,10,63,74,108,109,126,156,161,187,195,210)$ & 0.03 \\
\hline & CCID. $L_{\infty} \mathrm{IC}$ & 4 & $(62,70,154,187)$ & 0.03 \\
\hline & $\delta$ CCID. $L_{2}$ & 3 & $(9,74,156)$ & 0.02 \\
\hline & $\delta$ CCID. $L_{\infty}$ & 2 & $(62,154)$ & 0.01 \\
\hline & $\delta$ CCID. $L_{2} I C$ & 3 & $(9,74,156)$ & 0.05 \\
\hline & $\delta$ CCID. $L_{\infty} I C$ & 2 & $(62,154)$ & 0.02 \\
\hline \multirow{12}{*}{18} & SBS & 0 & - & 1.44 \\
\hline & Factor com & 1 & 75 & 2.83 \\
\hline & Factor id & 0 & - & 2.83 \\
\hline & $\mathrm{BO}$ & 2 & $(46,186)$ & 13.86 \\
\hline & CCID. $L_{2}$ & 6 & $(83,125,165,167,175,179)$ & 0.02 \\
\hline & CCID. $L_{\infty}$ & 1 & 145 & 0.03 \\
\hline & CCID. $L_{2} \mathrm{IC}$ & 1 & 179 & 0.02 \\
\hline & CCID. $L_{\infty} \mathrm{IC}$ & 1 & 184 & 0.02 \\
\hline & $\delta$ CCID. $L_{2}$ & 3 & $(59,138,205)$ & 0.02 \\
\hline & $\delta$ CCID. $L_{\infty}$ & 3 & $(65,108,205)$ & 0.01 \\
\hline & $\delta$ CCID. $L_{2} I C$ & 1 & 179 & 0.05 \\
\hline & $\delta$ CCID. $L_{\infty} I C$ & 1 & 184 & 0.02 \\
\hline \multirow{12}{*}{19} & SBS & 0 & 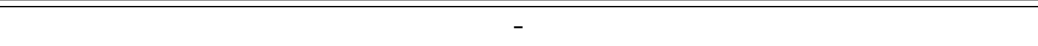 & 1.42 \\
\hline & Factor com & 0 & - & 3.25 \\
\hline & Factor id & 0 & - & 3.25 \\
\hline & $\mathrm{BO}$ & 0 & - & 5.26 \\
\hline & CCID. $L_{2}$ & 8 & $(61,71,125,130,142,186,194,195)$ & 0.02 \\
\hline & CCID. $L_{\infty}$ & 4 & $(56,75,130,141)$ & 0.01 \\
\hline & CCID. $L_{2} \mathrm{IC}$ & 8 & $(61,71,125,130,134,142,177,209)$ & 0.03 \\
\hline & CCID. $L_{\infty} \mathrm{IC}$ & 3 & $(75,130,141)$ & 0.02 \\
\hline & $\delta$ CCID. $L_{2}$ & 3 & $(71,142,186)$ & 0.02 \\
\hline & $\delta$ CCID.$L_{\infty}$ & 2 & $(75,141)$ & 0.01 \\
\hline & $\delta$ CCID. $L_{2} I C$ & 3 & $(59,108,195)$ & 0.05 \\
\hline & $\delta$ CCID. $L_{\infty} I C$ & 2 & $(71,142)$ & 0.02 \\
\hline
\end{tabular}




\begin{tabular}{|c|c|c|c|c|}
\hline ID & Method & Number & $\begin{array}{l}\text { Estimated } \\
\text { Locations }\end{array}$ & Time (s) \\
\hline \multirow{12}{*}{20} & SBS & 0 & - & 1.43 \\
\hline & Factor com & 0 & - & 3.45 \\
\hline & Factor id & 0 & - & 3.45 \\
\hline & $\mathrm{BO}$ & 0 & - & 5.12 \\
\hline & CCID. $L_{2}$ & 5 & $(96,101,104,139,140)$ & 0.01 \\
\hline & CCID. $L_{\infty}$ & 1 & 104 & 0.02 \\
\hline & CCID. $L_{2} \mathrm{IC}$ & 0 & - & 0.03 \\
\hline & CCID. $L_{\infty} \mathrm{IC}$ & 0 & - & 0.01 \\
\hline & $\delta$ CCID. $L_{2}$ & 1 & 104 & 0.02 \\
\hline & $\delta$ CCID.$L_{\infty}$ & 1 & 104 & 0.01 \\
\hline & $\delta$ CCID. $L_{2} I C$ & 0 & - & 0.05 \\
\hline & $\delta$ CCID.$L_{\infty} I C$ & 0 & - & 0.02 \\
\hline \multirow{12}{*}{21} & SBS & 0 & 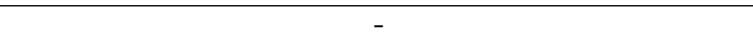 & 1.42 \\
\hline & Factor com & 0 & - & 2.31 \\
\hline & Factor id & 0 & - & 2.31 \\
\hline & $\mathrm{BO}$ & 0 & - & 5.18 \\
\hline & CCID. $L_{2}$ & 12 & $(7,47,62,97,100,112,115,126,146,160,169,183)$ & 0.01 \\
\hline & CCID. $L_{\infty}$ & 2 & $(7,160)$ & 0.02 \\
\hline & CCID. $L_{2} \mathrm{IC}$ & 11 & $(21,47,62,98,100,112,115,146,160,169,183)$ & 0.05 \\
\hline & CCID. $L_{\infty} \mathrm{IC}$ & 3 & $(7,160,183)$ & 0.03 \\
\hline & $\delta$ CCID. $L_{2}$ & 4 & $(7,62,115,183)$ & 0.02 \\
\hline & $\delta$ CCID.$L_{\infty}$ & 2 & $(7,160)$ & 0.01 \\
\hline & $\delta$ CCID. $L_{2} I C$ & 3 & $(62,125,183)$ & 0.05 \\
\hline & $\delta$ CCID. $L_{\infty} I C$ & 2 & $(7,183)$ & 0.02 \\
\hline \multirow{12}{*}{22} & SBS & 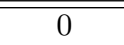 & - & 1.40 \\
\hline & Factor com & 0 & - & 2.36 \\
\hline & Factor id & 0 & - & 2.36 \\
\hline & $\mathrm{BO}$ & 0 & - & 5.10 \\
\hline & CCID. $L_{2}$ & 7 & $(61,63,101,105,130,179,209)$ & 0.01 \\
\hline & CCID. $L_{\infty}$ & 2 & $(73,135)$ & 0.03 \\
\hline & CCID. $L_{2} \mathrm{IC}$ & 8 & $(60,71,101,125,150,176,179,209)$ & 0.04 \\
\hline & CCID. $L_{\infty} \mathrm{IC}$ & 2 & $(73,135)$ & 0.02 \\
\hline & $\delta$ CCID. $L_{2}$ & 2 & $(61,179)$ & 0.02 \\
\hline & $\delta$ CCID.$L_{\infty}$ & 2 & $(73,135)$ & 0.01 \\
\hline & $\delta$ CCID. $L_{2} I C$ & 3 & $(60,101,179)$ & 0.05 \\
\hline & $\delta$ CCID. $L_{\infty} I C$ & 2 & $(73,135)$ & 0.02 \\
\hline \multirow{12}{*}{23} & SBS & 0 & - & 1.44 \\
\hline & Factor com & 1 & 174 & 3.11 \\
\hline & Factor id & 0 & - & 3.11 \\
\hline & $\mathrm{BO}$ & 0 & - & 5.22 \\
\hline & CCID. $L_{2}$ & 8 & $(60,69,128,145,155,176,194,207)$ & 0.02 \\
\hline & CCID. $L_{\infty}$ & 1 & 173 & 0.01 \\
\hline & CCID. $L_{2} \mathrm{IC}$ & 5 & $(60,69,173,194,207)$ & 0.06 \\
\hline & CCID. $L_{\infty} \mathrm{IC}$ & 1 & 173 & 0.03 \\
\hline & $\delta$ CCID. $L_{2}$ & 2 & $(60,145)$ & 0.02 \\
\hline & $\delta$ CCID. $L_{\infty}$ & 1 & 173 & 0.01 \\
\hline & $\delta$ CCID. $L_{2} I C$ & 2 & $(60,173)$ & 0.05 \\
\hline & $\delta$ CCID. $L_{\infty} I C$ & 1 & 173 & 0.02 \\
\hline
\end{tabular}


Table 12: The estimated number and location of the change-points for the resting state fMRI data set for Sparsified Binary Segmentation (SBS), Factor with common components (Factor com), Factor with idiosyncratic components (Factor id), Barnett \& Onnela (2016) (BO), and Cross-covariance isolate detect with the $L_{2}$ threshold (CCID. $L_{2}$ ), the $L_{\infty}$ threshold (CCID. $L_{\infty}$ ), the $L_{2}$ information criterion (CCID. $L_{2} I C$ ) and the $L_{\infty}$ information criterion (CCID. $L_{\infty} I C$ ). A $\delta$ in the name of a method indicates a minimum distance between change-points of 40 time points was enforced. The computational times for each method are also provided.

\begin{tabular}{|c|c|c|c|c|}
\hline ID & Method & Number & $\begin{array}{l}\text { Estimated } \\
\text { Locations }\end{array}$ & Time $(s)$ \\
\hline \multirow{13}{*}{1} & SBS & 1 & 72 & 142.90 \\
\hline & Factor com & 0 & - & 91.03 \\
\hline & Factor id & 0 & - & 91.03 \\
\hline & $\mathrm{BO}$ & 9 & $(11,35,66,83,116,139,178,193,211)$ & 352.57 \\
\hline & CCID. $L_{2}$ & 15 & $(22,41,73,85,90,124,134,145,190,198,206,217,257,264,275)$ & 9.23 \\
\hline & CCID. $L_{\infty}$ & 30 & $(18,31,51,72,74,78,86,118,121,123,125,129,131,134,138$ & \\
\hline & & & $146,181,186,188,190,193,195,198,201,207,228,256,260,262,266)$ & 17.42 \\
\hline & CCID. $L_{2} I C$ & 17 & $(14,25,38,52,64,73,90,108,124,132,146,169,194,222,236,253,276)$ & 56.42 \\
\hline & CCID. $L_{\infty} I C$ & 13 & $(18,44,64,97,118,138,158,181,197,216,236,254,266)$ & 54.69 \\
\hline & $\delta$ CCID. $L_{2}$ & 4 & $(90,145,190,257)$ & 0.02 \\
\hline & $\delta$ CCID. $L_{\infty}$ & 4 & $(18,86,146,256)$ & 0.01 \\
\hline & $\delta$ CCID. $L_{2} I C$ & 5 & $(38,108,194,236,276)$ & 0.05 \\
\hline & $\delta$ CCID. $L_{\infty} I C$ & 5 & $(18,64,138,181,254)$ & 0.02 \\
\hline \multirow{13}{*}{2} & SBS & 1 & 88 & 119.46 \\
\hline & Factor com & 0 & - & 92.24 \\
\hline & Factor id & 0 & - & 92.24 \\
\hline & $\mathrm{BO}$ & 8 & $(47,57,115,176,193,220,232,255)$ & 373.02 \\
\hline & CCID. $L_{2}$ & 14 & $(2,47,59,74,85,96,125,154,182,196,244,252,274,282)$ & 17.69 \\
\hline & CCID. $L_{\infty}$ & 22 & $(6,8,28,47,74,88,90,92,97,100,123,125,157,188,196,218$ & \\
\hline & & & $232,234,236,257,274,276)$ & 17.30 \\
\hline & CCID. $L_{2} I C$ & 8 & $(13,47,59,86,96,154,206,235)$ & 83.03 \\
\hline & CCID. $L_{\infty} I C$ & 7 & $(9,47,74,88,148,236,274)$ & 83.69 \\
\hline & $\delta$ CCID. $L_{2}$ & 5 & $(2,47,96,154,274)$ & 0.02 \\
\hline & $\delta$ CCID. $L_{\infty}$ & 4 & $(8,125,188,274)$ & 0.01 \\
\hline & $\delta$ CCID. $L_{2} I C$ & 4 & $(47,96,154,235)$ & 0.05 \\
\hline & $\delta$ CCID. $L_{\infty} I C$ & 4 & $(9,88,148,274)$ & 0.02 \\
\hline \multirow{12}{*}{3} & SBS & 2 & $(117,145)$ & 121.60 \\
\hline & Factor com & 1 & 40 & 90.54 \\
\hline & Factor id & 0 & - & 90.54 \\
\hline & $\mathrm{BO}$ & 9 & $(89,122,153,164,179,207,220,249,261)$ & 369.08 \\
\hline & CCID. $L_{2}$ & 12 & $(2,8,18,65,93,100,115,166,225,251,262,278)$ & 13.96 \\
\hline & CCID. $L_{\infty}$ & 18 & $(18,37,57,87,117,119,154,182,186,188,190,193,218,221,225,229,247,266)$ & 16.83 \\
\hline & CCID. $L_{2} I C$ & 11 & $(8,47,81,101,116,158,166,200,225,250,278)$ & 49.96 \\
\hline & CCID. $L_{\infty} I C$ & 3 & $(18,64,247)$ & 49.69 \\
\hline & $\delta$ CCID. $L_{2}$ & 4 & $(18,115,166,251)$ & 0.02 \\
\hline & $\delta$ CCID. $L_{\infty}$ & 3 & $(18,87,218)$ & 0.01 \\
\hline & $\delta$ CCID. $L_{2} I C$ & 4 & $(8,116,166,278)$ & 0.05 \\
\hline & $\delta$ CCID. $L_{\infty} I C$ & 3 & $(18,64,247)$ & 0.02 \\
\hline
\end{tabular}


bioRxiv preprint doi: https://doi.org/10.1101/2020.12.20.423696; this version posted June 8, 2021. The copyright holder for this preprint (which was not certified by peer review) is the author/funder, who has granted bioRxiv a license to display the preprint in perpetuity. It is made available under aCC-BY-NC-ND 4.0 International license.

\begin{tabular}{|c|c|c|c|c|}
\hline ID & Method & Number & $\begin{array}{l}\text { Estimated } \\
\text { Locations }\end{array}$ & Time (s) \\
\hline \multirow{13}{*}{4} & SBS & 1 & 173 & 114 \\
\hline & Factor com & 0 & - & 131.67 \\
\hline & Factor id & 0 & - & 131.67 \\
\hline & $\mathrm{BO}$ & 1 & 229 & 210.41 \\
\hline & CCID. $L_{2}$ & 9 & $(2,28,46,73,81,153,231,242,262)$ & 14.57 \\
\hline & CCID. $L_{\infty}$ & 22 & $(2,27,36,43,46,50,68,97,99,135,148,172,195,197,227,229$, & \\
\hline & & & $252,256,258,261,263,266)$ & 18.58 \\
\hline & CCID. $L_{2} I C$ & 11 & $(8,27,46,73,81,111,153,190,231,242,275)$ & 44.10 \\
\hline & CCID. $L_{\infty} I C$ & 3 & $(7,186,263)$ & 115.88 \\
\hline & $\delta$ CCID. $L_{2}$ & 4 & $(2,81,153,231)$ & 0.02 \\
\hline & $\delta$ CCID. $L_{\infty}$ & 3 & $(2,197,252)$ & 0.01 \\
\hline & $\delta$ CCID. $L_{2} I C$ & 5 & $(8,111,190,231,275)$ & 0.05 \\
\hline & $\delta$ CCID. $L_{\infty} I C$ & 3 & $(7,186,263)$ & 0.02 \\
\hline \multirow{13}{*}{5} & SBS & 1 & 189 & 115.89 \\
\hline & Factor com & 1 & 178 & 159.23 \\
\hline & Factor id & 0 & - & 159.23 \\
\hline & $\mathrm{BO}$ & 10 & $(30,63,84,107,141,184,204,235,248,263)$ & 440.72 \\
\hline & CCID. $L_{2}$ & 9 & $(6,20,65,95,115,172,221,231,269)$ & 10.61 \\
\hline & CCID. $L_{\infty}$ & 19 & $(6,12,22,42,67,70,88,110,112,127,158,177,180,182,207$ & \\
\hline & & & $234,236,257,278)$ & 13.07 \\
\hline & CCID. $L_{2} I C$ & 7 & $(6,74,117,172,197,207,267)$ & 29.89 \\
\hline & CCID. $L_{\infty} I C$ & 8 & $(22,68,107,112,159,182,224,263)$ & 49.26 \\
\hline & $\delta \mathrm{CCID} L_{2}$ & 4 & $(20,95,172,221)$ & 0.02 \\
\hline & $\delta$ CCID. $L_{\infty}$ & 4 & $(67,127,207,278)$ & 0.01 \\
\hline & $\delta$ CCID. $L_{2} I C$ & 5 & $(6,74,117,172,267)$ & 0.05 \\
\hline & $\delta$ CCID. $L_{\infty} I C$ & 4 & $(22,112,159,224)$ & 0.02 \\
\hline \multirow{13}{*}{6} & SBS & 2 & $(116,208)$ & 111.42 \\
\hline & Factor com & 1 & 94 & 139.20 \\
\hline & Factor id & 0 & - & 139.20 \\
\hline & $\mathrm{BO}$ & 10 & $(38,51,62,90,128,155,182,200,226,237)$ & 372.46 \\
\hline & CCID. $L_{2}$ & 13 & $(17,56,89,99,112,125,144,157,173,196,207,227,267)$ & 12.01 \\
\hline & CCID. $L_{\infty}$ & 22 & $(25,57,87,93,97,100,102,104,113,116,137,167,187,208,211$ & \\
\hline & & & $237,257,260,264,268,274277$ & 23.86 \\
\hline & CCID. $L_{2} I C$ & 8 & $(41,90,99,144,203,210,239,266)$ & 67.78 \\
\hline & CCID. $L_{\infty} I C$ & 9 & $(25,57,89,93,113,182,207,257,277)$ & 97.33 \\
\hline & $\delta$ CCID. $L_{2}$ & 5 & $(56,125,173,227,267)$ & 0.02 \\
\hline & $\delta$ CCID. $L_{\infty}$ & 5 & $(25,113,167,211,257)$ & 0.01 \\
\hline & $\delta$ CCID.$L_{2} I C$ & 5 & $(41,90,144,210,266)$ & 0.05 \\
\hline & $\delta$ CCID. $L_{\infty} I C$ & 4 & $(25,89,182,277)$ & 0.02 \\
\hline \multirow{12}{*}{7} & SBS & 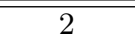 & $(102,151)$ & 121.45 \\
\hline & Factor com & 0 & - & 142.64 \\
\hline & Factor id & 0 & - & 142.64 \\
\hline & $\mathrm{BO}$ & 8 & $(50,66,103,115,139,169,239,264)$ & 396.44 \\
\hline & CCID. $L_{2}$ & 9 & $(2,61,70,117,173,207,234,245,275)$ & 13.94 \\
\hline & CCID. $L_{\infty}$ & 16 & $(18,21,47,71,88,114,128,157,169,194,219,237,257)$ & 16.82 \\
\hline & CCID. $L_{2} I C$ & 1 & 85 & 37.50 \\
\hline & CCID. $L_{\infty} I C$ & 4 & $(88,113,194,219)$ & 37.47 \\
\hline & $\delta$ CCID. $L_{2}$ & 4 & $(2,70,117,245)$ & 0.02 \\
\hline & $\delta$ CCID.$L_{\infty}$ & 5 & $(18,88,128,219,265)$ & 0.01 \\
\hline & $\delta$ CCID. $L_{2} I C$ & 1 & 85 & 0.05 \\
\hline & $\delta$ CCID. $L_{\infty} I C$ & 2 & $(113,219)$ & 0.02 \\
\hline
\end{tabular}


bioRxiv preprint doi: https://doi.org/10.1101/2020.12.20.423696; this version posted June 8, 2021. The copyright holder for this preprint (which was not certified by peer review) is the author/funder, who has granted bioRxiv a license to display the preprint in perpetuity. It is made available under aCC-BY-NC-ND 4.0 International license.

\begin{tabular}{|c|c|c|c|c|}
\hline ID & Method & Number & $\begin{array}{l}\text { Estimated } \\
\text { Locations }\end{array}$ & Time $(\mathrm{s})$ \\
\hline \multirow{13}{*}{8} & SBS & 1 & 130 & 121.22 \\
\hline & Factor com & 0 & - & 171.89 \\
\hline & Factor id & 0 & - & 171.89 \\
\hline & $\mathrm{BO}$ & 12 & $(12,36,61,84,96,121,131,192,221,238,251,265)$ & 409.98 \\
\hline & CCID. $L_{2}$ & 14 & $(15,53,87,98,126,134,141,150,167,190,206,235,264,282)$ & 9.83 \\
\hline & CCID. $L_{\infty}$ & 23 & $(2,24,53,68,71,73,98,116,138,141,144,147,151,173,188$ & \\
\hline & & & $206,225,227,259,261,264,273,278)$ & 20.29 \\
\hline & CCID. $L_{2} I C$ & 7 & $(126,150,186,203,214,234,264)$ & 36.68 \\
\hline & CCID. $L_{\infty} I C$ & 4 & $(42,196,237,278)$ & 84.21 \\
\hline & $\delta$ CCID. $L_{2}$ & 4 & $(53,98,190,235)$ & 0.02 \\
\hline & $\delta$ CCID. $L_{\infty}$ & 3 & $(2,53,188)$ & 0.01 \\
\hline & $\delta$ CCID $\cdot L_{2} I C$ & 2 & $(126,234)$ & 0.05 \\
\hline & $\delta$ CCID. $L_{\infty} I C$ & 4 & $(42,196,237,278)$ & 0.02 \\
\hline \multirow{13}{*}{9} & SBS & 1 & 212 & 110.36 \\
\hline & Factor com & 1 & 236 & 139.03 \\
\hline & Factor id & 0 & - & 139.03 \\
\hline & $\mathrm{BO}$ & 10 & $(34,59,81,124,147,168,200,215,244,257)$ & 365.12 \\
\hline & CCID. $L_{2}$ & 8 & $(68,79,145,189,201,235,246,270)$ & 11.36 \\
\hline & CCID. $L_{\infty}$ & 19 & $(8,11,35,57,60,87,113,134,137,142,172,188,217,234,246$, & \\
\hline & & & $269,271,273,277)$ & 15.39 \\
\hline & CCID. $L_{2} I C$ & 9 & $(29,42,68,87,127,171,189,215,264)$ & 33.94 \\
\hline & CCID. $L_{\infty} I C$ & 5 & $(51,86,177,225,269)$ & 57.17 \\
\hline & $\delta$ CCID. $L_{2}$ & 3 & $(79,145,189)$ & 0.02 \\
\hline & $\delta$ CCID.$L_{\infty}$ & 4 & $(11,87,142,246)$ & 0.01 \\
\hline & $\delta$ CCID. $L_{2} I C$ & 6 & $(29,87,127,171,215,264)$ & 0.05 \\
\hline & $\delta$ CCID. $L_{\infty} I C$ & 4 & $(86,177,225,269)$ & 0.02 \\
\hline \multirow{13}{*}{10} & SBS & 1 & 202 & 109.35 \\
\hline & Factor com & 0 & - & 178.42 \\
\hline & Factor id & 0 & - & 178.42 \\
\hline & $\mathrm{BO}$ & 11 & $(33,69,97,112,129,149,161,193,211,237,249)$ & 363.08 \\
\hline & CCID. $L_{2}$ & 12 & $(2,42,65,97,108,149,166,200,215,235,251,282)$ & 11.47 \\
\hline & CCID. $L_{\infty}$ & 28 & $(17,43,45,67,74,77,97,117,119,148,159,162,164,167,170$ & \\
\hline & & & $172,186,188,198,200,202,205,207,233,235,237,256,282)$ & 16.06 \\
\hline & CCID. $L_{2} I C$ & 10 & $(17,41,66,82,117,144,166,234,251,276)$ & 42.29 \\
\hline & CCID. $L_{\infty} I C$ & 12 & $(17,38,58,65,107,119,138,157,188,220,256,282)$ & 101.19 \\
\hline & $\delta$ CCID. $L_{2}$ & 5 & $(2,42,108,215,282)$ & 0.02 \\
\hline & $\delta$ CCID. $L_{\infty}$ & 5 & $(17,67,117,186,282)$ & 0.01 \\
\hline & $\delta$ CCID. $L_{2} I C$ & 5 & $(17,82,144,234,276)$ & 0.05 \\
\hline & $\delta$ CCID. $L_{\infty} I C$ & 5 & $(17,58,119,188,282)$ & 0.02 \\
\hline
\end{tabular}


Table 13: The distribution of $\hat{N}-N$ and the scaled Hausdorff distance over 100 simulated data sequences from Simulations 1, 3 , and 5 , with the noise following the $t_{5}$ distribution, for the Cross-covariance isolate detect with the $L_{2}$ threshold (CCID. $L_{2}$ ), the $L_{\infty}$ threshold (CCID. $L_{\infty}$ ), the $L_{2}$ information criterion (CCID. $L_{2} I C$ ) and the $L_{\infty}$ information criterion (CCID. $L_{\infty} I C$ ). The computational times are also provided.

\begin{tabular}{|c|c|c|c|c|c|c|c|c|c|c|}
\hline \multirow[b]{2}{*}{ Simulation data } & \multirow[b]{2}{*}{ Method } & \multirow[b]{2}{*}{$\leq-3$} & \multicolumn{4}{|c|}{$\hat{N}-N$} & \multirow[b]{2}{*}{2} & \multirow[b]{2}{*}{$\geq 3$} & \multirow[b]{2}{*}{$d_{H}$} & \multirow[b]{2}{*}{ Time (s) } \\
\hline & & & -2 & -1 & 0 & 1 & & & & \\
\hline \multirow{8}{*}{1} & SBS & - & - & - & 100 & 0 & 0 & 0 & - & 2.82 \\
\hline & Factor com & - & - & - & 100 & 0 & 0 & 0 & - & 16.62 \\
\hline & Factor id & - & - & - & 95 & 5 & 0 & 0 & - & 16.62 \\
\hline & $\mathrm{BO}$ & - & - & - & 85 & 15 & 0 & 0 & - & 7.66 \\
\hline & CCID. $L_{2}$ & - & - & - & 39 & 21 & 25 & 15 & - & 0.28 \\
\hline & CCID. $L_{\infty}$ & - & - & - & 28 & 23 & 21 & 28 & - & 0.36 \\
\hline & CCID. $L_{2} I C$ & - & - & - & 97 & 1 & 2 & 0 & - & 0.42 \\
\hline & CCID. $L_{\infty} I C$ & - & - & - & 98 & 1 & 1 & 0 & - & 0.39 \\
\hline \multirow{8}{*}{3} & SBS & 5 & 5 & 16 & 74 & 0 & 0 & 0 & 0.50 & 3.90 \\
\hline & Factor com & 100 & 0 & 0 & 0 & 0 & 0 & 0 & 4 & 18.22 \\
\hline & Factor id & 73 & 11 & 10 & 6 & 0 & 0 & 0 & 2.96 & 18.22 \\
\hline & $\mathrm{BO}$ & 3 & 2 & 13 & 57 & 22 & 3 & 0 & 0.55 & 39.87 \\
\hline & CCID. $L_{2}$ & 0 & 0 & 12 & 59 & 21 & 6 & 2 & 0.31 & 0.17 \\
\hline & CCID. $L_{\infty}$ & 0 & 0 & 4 & 44 & 30 & 16 & 6 & 0.35 & 0.18 \\
\hline & CCID. $L_{2} I C$ & 1 & 1 & 12 & 74 & 7 & 5 & 0 & 0.31 & 0.56 \\
\hline & CCID. $L_{\infty} I C$ & 1 & 5 & 11 & 76 & 3 & 4 & 0 & 0.37 & 0.32 \\
\hline \multirow{8}{*}{5} & SBS & 69 & 26 & 1 & 4 & 0 & 0 & 0 & 3.03 & 3.95 \\
\hline & Factor com & 100 & 0 & 0 & 0 & 0 & 0 & 0 & 4 & 19.32 \\
\hline & Factor id & 16 & 11 & 2 & 71 & 0 & 0 & 0 & 0.89 & 19.32 \\
\hline & $\mathrm{BO}$ & 60 & 21 & 8 & 6 & 4 & 1 & 0 & 2.89 & 24.26 \\
\hline & CCID. $L_{2}$ & 0 & 0 & 0 & 57 & 24 & 12 & 7 & 0.21 & 0.18 \\
\hline & CCID. $L_{\infty}$ & 0 & 2 & 6 & 37 & 21 & 13 & 21 & 0.45 & 0.21 \\
\hline & CCID. $L_{2} I C$ & 15 & 10 & 2 & 53 & 7 & 10 & 3 & 0.94 & 0.65 \\
\hline & CCID. $L_{\infty} I C$ & 24 & 19 & 1 & 44 & 5 & 6 & 1 & 1.38 & 0.46 \\
\hline
\end{tabular}

B Figures showing the results of SBS, Factor and BO for the fMRI data sets 
bioRxiv preprint doi: https://doi.org/10.1101/2020.12.20.423696; this version posted June 8, 2021. The copyright holder for this preprint (which was not certified by peer review) is the author/funder, who has granted bioRxiv a license to display the preprint in perpetuity. It is made available under aCC-BY-NC-ND 4.0 International license.

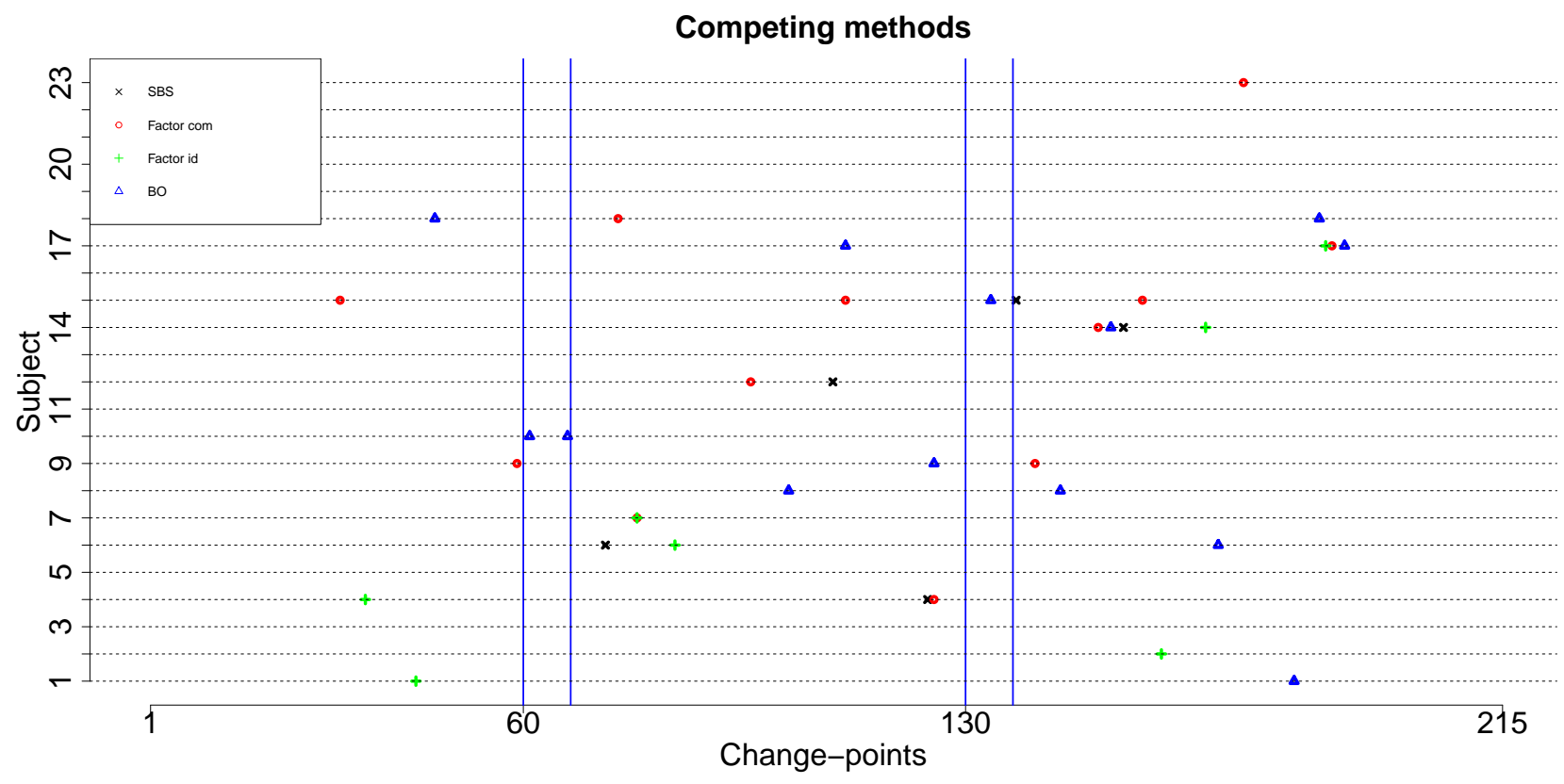

Figure 14: The detected change-points for the 4 ROI and heart rate data set using SBS, Factor, and BO. The y-axis depicts the subject number while the $\mathrm{x}$-axis shows the change-point location times. The blue vertical lines indicate the times of the showing and of the removal of the visual cues.

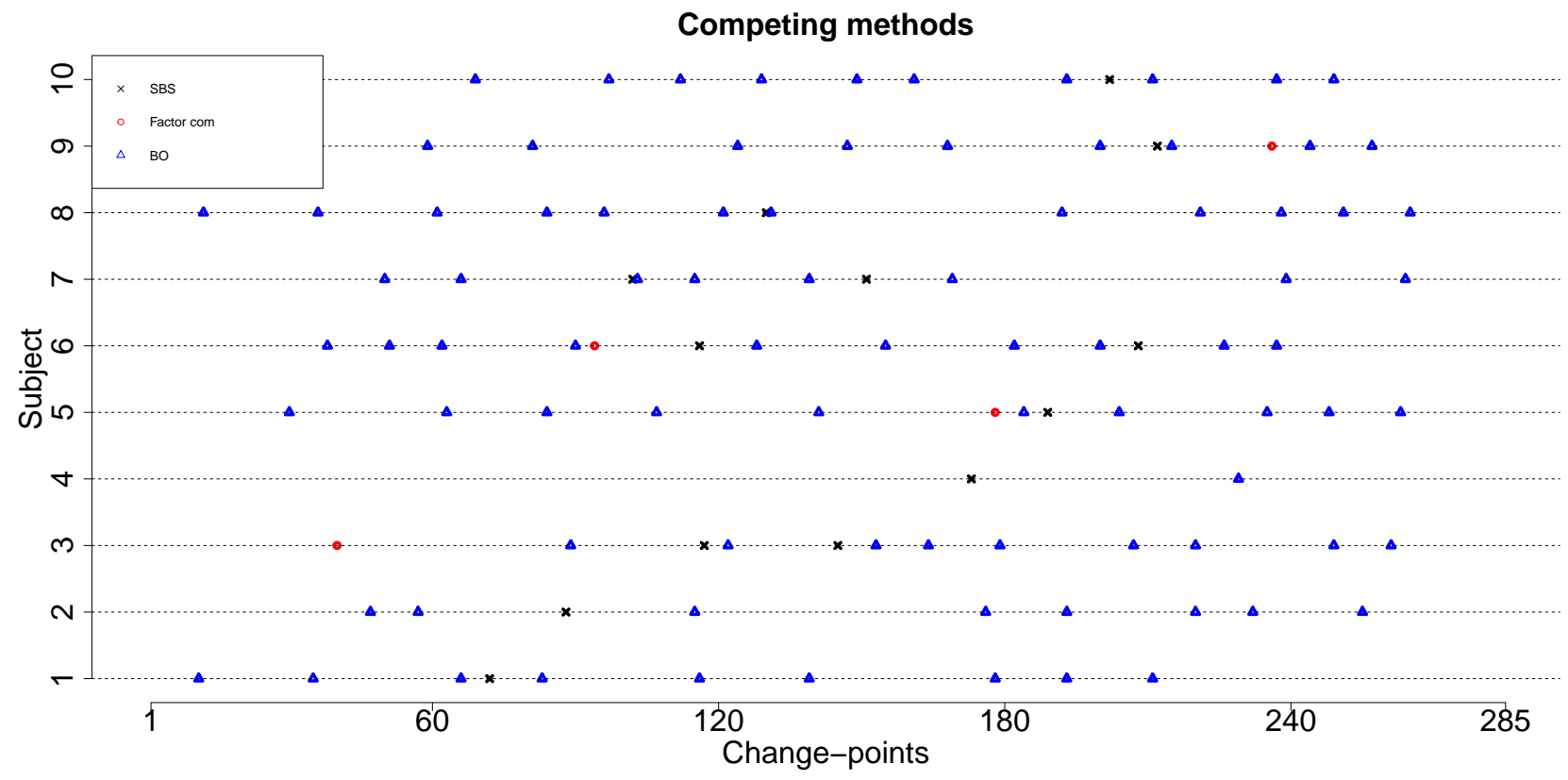

Figure 15: The detected change-points for the resting state fMRI data set using SBS, Factor com, and BO. The results for Factor idiosyncratic are not presented because this method does not detect any change-points for all subjects. The $\mathrm{y}$-axis depicts the subject number while the $\mathrm{x}$-axis shows the change-point location times. 